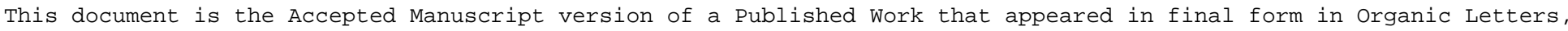

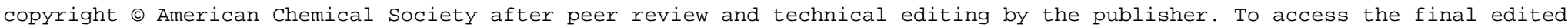
and published work see https://pubs.acs.org/doi/10.1021/acs.orglett.8b00337.

\title{
Iridium- and Rhodium-Catalyzed Directed C-H Heteroarylation of Benzaldehydes with Benziodoxolone Hypervalent lodine Reagents
}

\author{
Erwann Grenet and Jérôme Waser*
}

Laboratory of Catalysis and Organic Synthesis, Institute of Chemical Sciences and Engineering, Ecole Polytechnique Fedérale de Lausanne, EPFL SB ISIC LCSO, BCH 4306, 1015 Lausanne, Switzerland

Supporting Information Placeholder
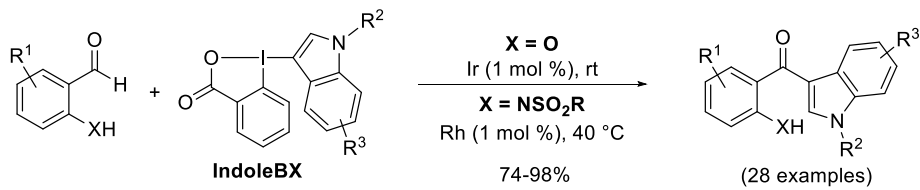

\begin{abstract}
The C-H heteroarylation of benzaldehydes with indoles and pyrroles was realized using the benziodoxolone hypervalent iodine reagents Indole- and Pyrrole- BX. Functionalization of the aldehyde C-H bond using either an ortho-hydroxy or amino directing group and catalyzed by an iridium or a rhodium complex allowed the synthesis of salicyloylindoles and (2-sulfonamino)benzoylindoles, respectively with good to excellent yields (74 to 98\%). This new transformation could be carried out under mild conditions ( $\mathrm{rt}$ to $40^{\circ} \mathrm{C}$ ) and tolerated a broad range of functionalities, such as ethers, halogens, carbonyls or nitro groups.
\end{abstract}

Indoles and pyrroles are ubiquitous in medicinal chemistry and natural products. ${ }^{1}$ Aryl indolyl ketones have attracted strong interest due to their interesting biological activities, in particular through interactions with the cannabinoid receptor. ${ }^{2}$ Among them, the subclass in which the aryl moiety wears a hydroxy or an amino group in ortho position to the carbonylshowed in addition diverse biological activities (Figure 1). Polymethoxylated indole derivatives 1 were cytotoxic against KB, MKN45, MCF-7 and colon HT-29 cells. ${ }^{3}$ 3-(2-Aminobenzoyl)indole 2 led to VEGFR-2 inhibition. ${ }^{4}$ Furthermore, the salicoyl pyrrole core is present in bhimamycins $\mathbf{3}$, antibiotic natural products isolated from the bacteria Streptomyces $s p .^{5}$
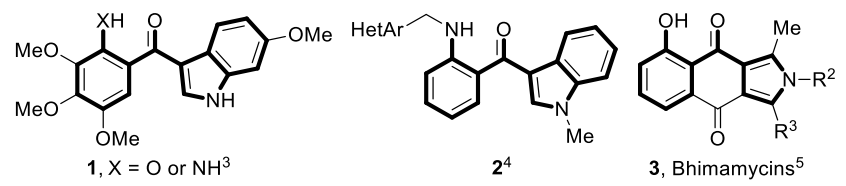

Figure 1. Synthetic and natural bioactive compounds with a 2-hydroxy or 2-amino benzoyl indole or pyrrole core

Due to their occurrence in biologically active compounds, the efficient synthesis of 3-benzoyl-indoles or -pyrroles is important. The most straightforward approach is based on the innate reactivity of the heterocycles as nucleophiles combined with an electrophilic acylation reagent (Scheme 1a). However, Friedel-Crafts acylation of indoles under standard conditions usually leads to a complex mixture of mono-, di-acylated and oligomerization products due to their high electron density. ${ }^{1 a}$ This issue can be partially resolved by the introduction of elec- tron-withdrawing protecting groups, the use of milder Lewis acids $\left(\mathrm{Et}_{2} \mathrm{AlCl}^{6 \mathrm{a}-\mathrm{b}}\right.$, imidazolium chloroaluminate ${ }^{6 \mathrm{c}}$ or $\left.\mathrm{ZrCl}_{4}{ }^{6 \mathrm{~d}}\right)$, reaction with nitrilium salts ${ }^{7}$ or the use of hexafluoroisopropanol as solvent. ${ }^{8}$ Furthermore, the nucleophilicity of the heterocycles can be enhanced by conversion into Grignard or other organometallic reagents, which can then be added directly to electrophiles or used in metal-catalyzed cross-couplings. ${ }^{9}$ Recently, direct $\mathrm{C}-\mathrm{H}$ acylation catalyzed by transition metals has also been reported. ${ }^{10}$ The synthesis of 3 -salicyloyl indoles was either not reported in these works, or required the protection/deprotection of the hydroxy group. Therefore, developing a more direct access to these compounds would be highly desirable. In this respect, only limited success has been achieved by the ring-opening of chromones, ${ }^{11,12}$ 1,3-dipolar cycloaddition, followed by decarboxylation, ${ }^{13}$ and alkylation of indoles with nitroolefins, followed by oxidative C-C bond cleavage. ${ }^{14}$

Scheme 1. Classical vs Umpolung approaches for the synthesis of 3-arylcarbonyl indoles

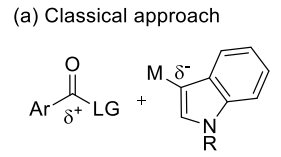

(b) Umpolung approach (this work)

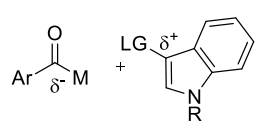

$$
\begin{gathered}
\begin{array}{c}
\text { mild conditions } \\
\text { regioselective } \\
\text { OH/NHR tolerated }
\end{array} \\
\hline M=I r, R h \\
L G=I(I I I)
\end{gathered}
$$

As an alternative strategy, we envisaged an Umpolung pathway involving a nucleophilic acyl metal intermediate generated 
via $\mathrm{C}_{\mathrm{sp} 2}-\mathrm{H}$ activation of an aldehyde ${ }^{15}$ and an electrophilic indole (Scheme 1b). Concerning the nucleophilic partners, benzaldehydes substituted with oxygen or nitrogen heteroatoms in ortho position are privileged substrates, as the directing group is necessary for $\mathrm{C}-\mathrm{H}$ activation under mild conditions. ${ }^{15 a}$ As electrophilic partners, we considered hypervalent iodine(III) reagents, which have been extensively used as Umpolung reagents in numerous transformations. ${ }^{16}$ In fact, both aryl iodoniums and ethynylbenziodoxolones (EBX) have been used to introduce phenyl or alkyne derivatives via cross-coupling on salicylaldehydes ${ }^{17}$ However, there are only few methods for the synthesis of indole-and pyrrole-based iodonium salts. ${ }^{18}$ Furthermore, due to their limited stability, these compounds have found only very limited use in transition metal catalysis. Recently, our's and Yoshikai's group reported the synthesis of bench-stable indole- and pyrrole-benziodoxolones (indoleBX and pyrroleBX).${ }^{19} \mathrm{We}$ further demonstrated that the new indoleand pyrrole- BX reagents could be used for directed $\mathrm{C}-\mathrm{H}$ functionalization with rhodium or ruthenium catalysts, whereas iodonium salts were not successful. ${ }^{19 a}$ Herein, we report the $\mathrm{C}-\mathrm{H}$ functionalization of 2-hydroxy and 2-amino benzaldehydes derivatives with Indole- and Pyrrole-BX reagents using either iridium or rhodium catalysts to give access to important indole and pyrrole building blocks.

We initiated the studies on $\mathrm{C}-\mathrm{H}$ indolation with the optimization of the reaction conditions for the coupling of salicylaldehyde $4 \mathbf{a}$ with Me-indoleBX 5a (Table 1). While $\left[\mathrm{RhCp} * \mathrm{Cl}_{2}\right]_{2}$ as catalyst gave only traces of the desired product $\mathbf{6 a}$ (Table 1, entry 1$)$, we were pleased to see that the use of $\left[\mathrm{IrCp}^{*} \mathrm{Cl}_{2}\right]_{2}$ led to formation of $\mathbf{6 a}$ in excellent $91 \%$ yield in the presence of cesium acetate at room temperature (Table 1, entry 2). This is the first example of the use of an iridium catalyst with IndoleBX reagents. Furthermore, the reaction did not require any particular precautions concerning the presence of water or oxygen. Complete conversion was reached after 10 minutes at room temperature. Cheaper potassium acetate could also be used to give $90 \%$ yield of the ketone product $\mathbf{6 a}$ (Table 1, entry 3). Variation of the amount of KOAc revealed that a superstoichiometric amount was not necessary (Table 1, entry 4). However, the yield decreased when the base was used in a substoichiometric quantity (Table 1, entry 5) and without base only $56 \%$ yield was obtained, even if full conversion was still observed (Table 1, entry 6). Finally, we were able to reduce the catalyst loading to $1 \mathrm{~mol}$ $\%$ without significant change in yield, demonstrating the robustness of the catalyst (Table 1 , entry 7 ). A scale-up to 1.20 mmol allowed us to decrease the iridium catalyst loading to 0.5 mol \%, giving $93 \%$ yield of $\mathbf{6 a}$ in the same reaction time (Table 1 , entry 8). Control experiments indicated that the transition metal complex is essential for the reaction. ${ }^{20}$ When 3-bromo-1methylindole and 3-iodo-1-methylindole were used as reagents, the desired compounds were obtained in 59\% and 53\% yield respectively, but only after heating over night at $80{ }^{\circ} \mathrm{C} .{ }^{20}$ This result further highlights the exceptional reactivity of IndoleBX reagents.

The scope of the reaction was then studied (Scheme 2). The effect of substituents in the para position to the hydroxy group was examined first (6a-i). In terms of electronic effect, both electron-donating alkyl and ether groups (6b and $\mathbf{6 c}$ ) and electron-withdrawing halogens, aldehyde and nitro groups (6d-i) were well tolerated. The exclusive formation of product $\mathbf{6 h}$ starting from a bis-formylated benzene confirmed the require- ment of a directing group for $\mathrm{C}-\mathrm{H}$ activation. ${ }^{21}$ In term of substitution pattern of the benzene ring, a methoxy group was tolerated also in all other positions $(\mathbf{6} \mathbf{j}-\mathbf{l})$.

Table 1. Optimization studies ${ }^{\mathrm{a}}$

$\begin{array}{cccc} & & \\ 1 & {\left[\mathrm{RhCp}^{*} \mathrm{Cl}_{2}\right]_{2}} & \mathrm{CsOAc}(1.2) & \text { traces }^{\mathrm{c}} \\ 2 & {\left[\mathrm{IrCp}^{*} \mathrm{Cl}_{2}\right]_{2}} & \mathrm{CsOAc}(1.2) & 91 \\ 3 & {\left[\mathrm{IrCp}^{*} \mathrm{Cl}_{2}\right]_{2}} & \mathrm{KOAc}(1.2) & 90 \\ 5 & {\left[\mathrm{IrCp}^{*} \mathrm{Cl}_{2}\right]_{2}} & \operatorname{KOAc}(1.0) & 90 \\ 6 & {\left[\mathrm{IrCp}^{*} \mathrm{Cl}_{2}\right]_{2}} & \operatorname{KOAc}(0.5) & 86 \\ 7 & {\left[\mathrm{IrCp}^{*} \mathrm{Cl}_{2}\right]_{2}} & - & 56 \\ 8 & {\left[\mathrm{IrCp}^{*} \mathrm{Cl}_{2}\right]_{2}} & \operatorname{KOAc}(1.0) & 94^{\mathrm{d}} \\ & {\left[\mathrm{IrCp}^{*} \mathrm{Cl}_{2}\right]_{2}} & \operatorname{KOAc}(1.0) & 93^{\mathrm{e}}\end{array}$

aReactions conditions: 4a (0.05 mmol), 5a (0.05 mmol), $\left[\mathrm{IrCp} * \mathrm{Cl}_{2}\right]_{2}(3 \mathrm{~mol} \%)$, base, methanol $(0.5 \mathrm{~mL})$ at $\mathrm{rt}$ for $10 \mathrm{~min}$. bIsolated yield after column chromatography. ${ }^{\mathrm{c} R e a c t i o n}$ performed at $80{ }^{\circ} \mathrm{C} .{ }^{\mathrm{d}}\left[\mathrm{IrCp} * \mathrm{Cl}_{2}\right]_{2}(1 \mathrm{~mol} \%) .{ }^{\text {eY }}$ ield at a $1.20 \mathrm{mmol}$ scale with $0.5 \mathrm{~mol} \%\left[\mathrm{IrCp}^{*} \mathrm{Cl}_{2}\right]_{2}$

Scheme 2. Ir(III)-catalyzed C-H indolation of 2-hydroxybenzaldehydes. ${ }^{\text {a,b }}$
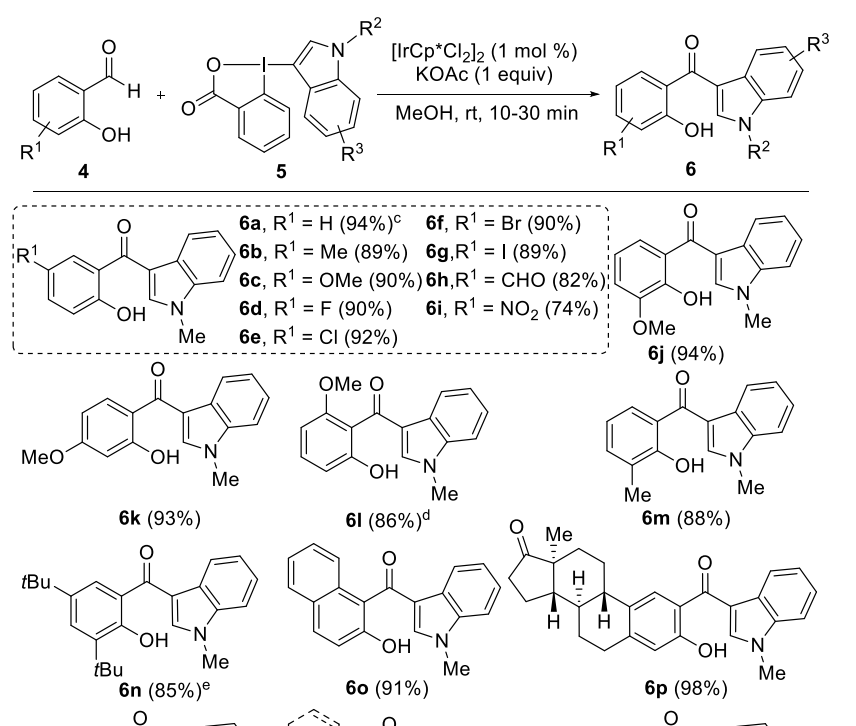

$6 \mathrm{~m}(88 \%)$
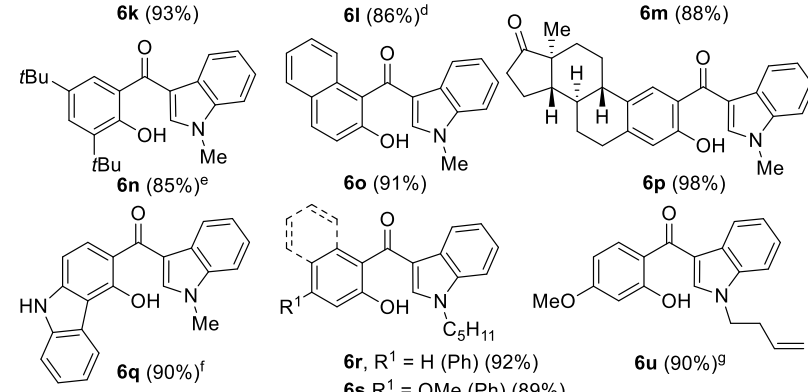

$6 r, R^{1}=H(P h)(92 \%)$
$6 s, R^{1}=O M e(P h)(89 \%)$

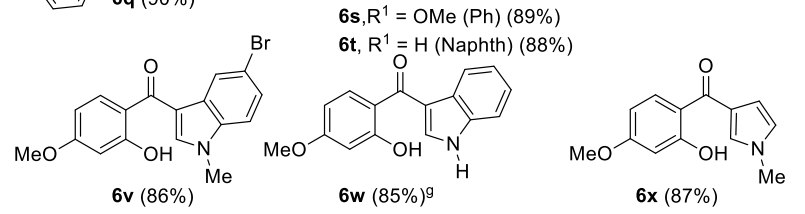

aReactions conditions: 4 (0.15 mmol), 5 (0.15 mmol), $\left[\mathrm{IrCp} * \mathrm{Cl}_{2}\right]_{2}(1 \mathrm{~mol} \%)$, KOAc $(0.15 \mathrm{mmol})$, methanol $(1.5 \mathrm{~mL})$ at rt for $10 \mathrm{~min}$. ${ }^{\mathrm{b}}$ Isolated yield after column chromatography. ${ }^{\mathrm{c}} 93 \%$ 
yield for a reaction at $1.20 \mathrm{mmol}$ scale. ${ }^{\mathrm{d}} 30 \mathrm{~min} .{ }^{\mathrm{e}} 1 \mathrm{~h}$. ${ }^{\mathrm{f}}$ Reaction performed at $50{ }^{\circ} \mathrm{C}$ for $2 \mathrm{~h}$. ${ }^{g}$ Reaction performed at $70{ }^{\circ} \mathrm{C}$ for $2 \mathrm{~h}$.

A longer reaction time of 30 minutes was observed only in case of 2-hydroxy-6-methoxybenzaldehyde $\mathbf{4 1}$ to give $\mathbf{6 1}$ in $86 \%$ yield. Alkyl-substituted products $\mathbf{6 m}$ and $\mathbf{6 n}$ were also obtained in good yields. Naphthalene and estrone-derivatives $\mathbf{6 0}$ and $\mathbf{6 p}$ were isolated in excellent $91 \%$ and $98 \%$ yields respectively. Pentacyclic compound $\mathbf{6 q}$ wearing a carbazole heterocycle was obtained in $90 \%$ yield. Modification of the hypervalent iodine reagent was then investigated. Changing the $N$-substitution from methyl to pentyl or butenyl delivered indoles $6 \mathbf{r}-\mathbf{u}$. The corresponding $O$-methylated compounds are reported synthetic cannabinoids. ${ }^{22}$ A bromo substituent on the benzene ring was well tolerated $(\mathbf{6 v})$. The $N$-H free compound $\mathbf{6 w}$ could be also obtained with complete regioselectivity in $85 \%$ yield. For products $\mathbf{6 u}$ and $\mathbf{6 w}$, it was necessary to perform the reaction at 70 ${ }^{\circ} \mathrm{C}$ to reach full conversion. Importantly, the method could be extended to the synthesis of pyrrole $\mathbf{6 x}$ using a PyrroleBX as reagent.

We then turned to the use of nitrogen-based directing groups for the activation of the aldehyde $\mathrm{C}-\mathrm{H}$ bond. In the case of 2aminobenzaldehyde $7 \mathbf{a}$ bearing a $N$-tosyl directing group a $\mathrm{Rh}$ (III) dimer catalyst proved to be as effective as the $\operatorname{Ir}(\mathrm{III})$ catalyst (Scheme 3 ), in contrast to what had been observed with salicylaldehyde 4a (Table 1). The reaction was best run in $\mathrm{MeOH}$ at $40{ }^{\circ} \mathrm{C}$ during $4 \mathrm{~h} .{ }^{20}$ Several $N$-sulfonyl groups (tosyl, para-nosyl and mesyl) could be used to direct the $\mathrm{C}-\mathrm{H}$ functionalization, giving products $\mathbf{8 a - c}$ in $80-88 \%$ yield. In contrast, a $N$-boc directing group was inefficient for this transformation (result not shown). The reaction could be perform in the presence of a chlorine atom on the phenyl ring affording the product 8d with $91 \%$ yield. The thiophene-derivatived compound $8 \mathbf{8}$ was isolated in an excellent $90 \%$ yield. This rhodium-catalyzed reaction also tolerated a $N$-butenyl substituent or a bromo group on the indole benzene ring, giving the desired products $8 \mathbf{f}$ and $\mathbf{8 g}$ in $79 \%$ and $82 \%$ yields, respectively.

Scheme 3. Rh(III)-catalyzed C-H indolation of 2-sulfonylaminobenzaldehydes. ${ }^{\text {a,b }}$

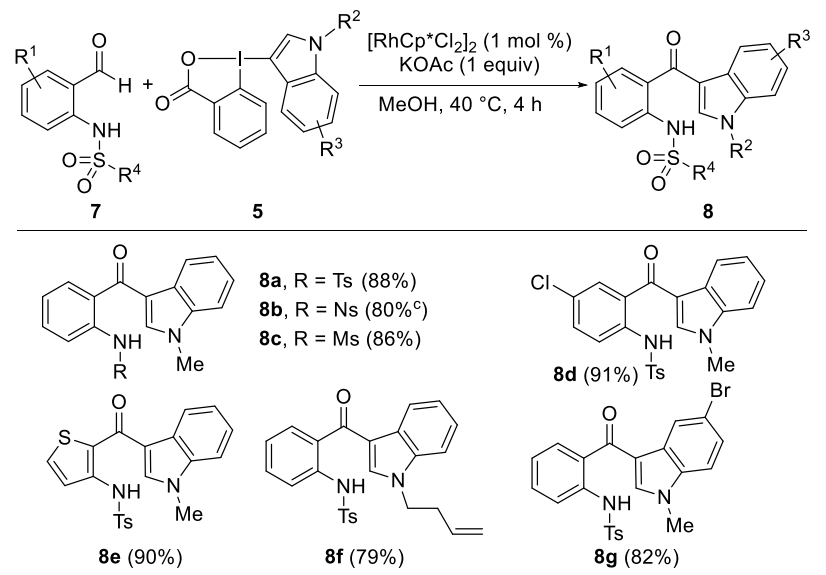

aReactions conditions: 7 (0.15 mmol), 5 (0.15 mmol), $\left[\mathrm{RhCp} * \mathrm{Cl}_{2}\right]_{2}(1 \mathrm{~mol} \%), \mathrm{KOAc}(0.15 \mathrm{mmol})$, methanol $(1.5 \mathrm{~mL})$ at $40{ }^{\circ} \mathrm{C}$ for $4 \mathrm{~h}$. ${ }^{\mathrm{b}}$ Isolated yield after column chromatography. ${ }^{\mathrm{c}} 80 \%$ yield for a reaction at $0.80 \mathrm{mmol}$ scale.

The directing groups were not only useful for allowing $\mathrm{C}-\mathrm{H}$ functionalization under mild conditions (see Supporting Infor- mation for a mechanism proposal), ${ }^{15 \mathrm{a}, 17 \mathrm{c}}$ they also served as handles for further modifications (Scheme 4). For example, phenol 6a was quantitatively transformed into the corresponding triflate 9 by reaction with triflic anhydride. Suzuki-Miyaura crosscoupling with phenyl boronic acid gave then biphenyl derivative 10 in $89 \%$ yield over 2 steps. Alternatively, the directing group could be fully removed by a palladium-catalyzed reduction of the triflate to furnish 3-benzoylindole 11. From $N$-sulfonylphenyl substituted ketones $\mathbf{8 a}$ and $\mathbf{8 b}$, an iodine-mediated oxidative C-2 amination generated tetracyclic indolo$[2,3 b]$ quinolinone 12a and $\mathbf{1 2} \mathbf{b}$ in quantitative yield for tosyl $\mathbf{8 a}$ and $89 \%$ yield for nosyl $\mathbf{8 b} .^{22}$ The nosyl protecting group was synthetically especially useful, as it could be removed in presence of thiophenol, either on the cyclized product $\mathbf{1 2 b}$ or the indolation product $\mathbf{8 b}$ to give the $N$-H free heterocycles $\mathbf{1 3}$ and 14.

Scheme 4. Product modifications

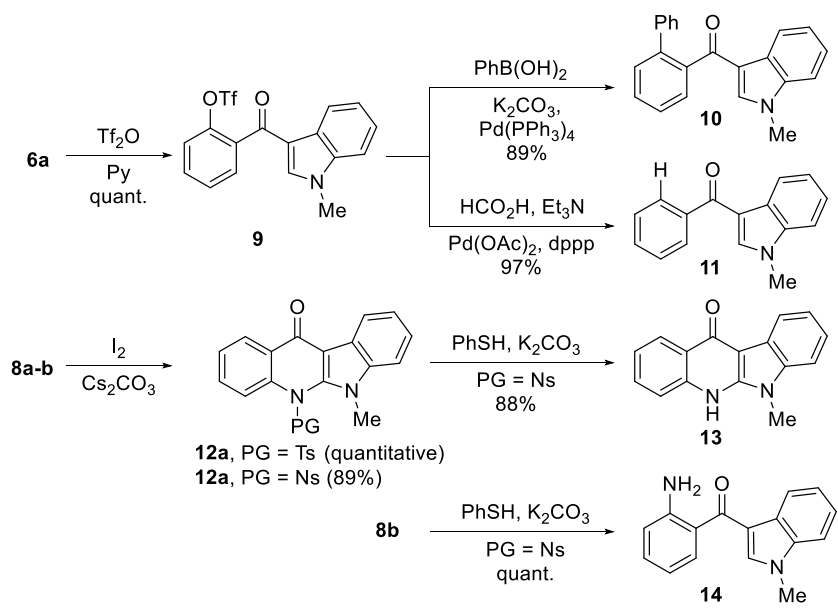

In summary, we have reported the first example of aldehyde $\mathrm{C}-\mathrm{H}$ heteroarylation giving highly useful indole and pyrrole building blocks. The reaction proceeded under mild, neutral conditions using either an alcohol or a sulfonylamide directing group with an iridium or a rhodium catalyst, respectively and the cyclic hypervalent iodine reagents Indole- and PyrroleBX. This represented also the first use of an iridium catalyst with Indole- and PyrroleBX as reagents. As the reaction tolerated a broad range of functional groups and the obtained versatile indole and pyrrole building blocks could be easily further modified, the method is expected to be highly useful in synthetic and medicinal chemistry.

\section{ASSOCIATED CONTENT}

\section{Supporting Information}

Detailed optimization tables, experimental procedures, analytical data for all compounds and copies of the NMR spectra for new compounds are available free of charge on the ACS Publications website.

\section{AUTHOR INFORMATION}

\section{Corresponding Author}

*e-mail: jerome.waser@epfl.ch

\section{Notes}

The authors declare no competing financial interest. 


\section{ACKNOWLEDGMENT}

This work is supported by the ERC starting grant 334840 , iTools4MC and EPFL.

\section{REFERENCES}

${ }^{1}$ (a) Sundberg, R. J. The Chemistry of Indoles; Academic Press: New York, 1970; (b) Gribble, G. W. Indole Ring Synthesis: From Natural Products to Drug Discovery. Wiley: 2016; (c) Zhang, M.-Z.; Chen, Q.; Yang, G.-F. Eur. J. Med. Chem. 2015, 89, 421.

2 (a) Pertwee, R.; Griffin, G.; Fernando, S.; Li, X.; Hill, A.; Makriyannis, A., Life Sci. 1995, 56, 1949; (b) Patwardhan, A. M.; Jeske, N. A.; Price, T. J.; Gamper, N.; Akopian, A. N.; Hargreaves, K. M., Proc. Natl. Acad. Sci. U. S. A. 2006, 103, 11393; (c) Metwally, M. A.; Shaaban, S.; Abdel-Wahab, B.-F.; El-Hiti, G. A. Curr. Org. Chem. 2009, 13, 1475 .

${ }^{3}$ (a) Lee, H.-Y.; Chang, C.-Y.; Lai, M.-J.; Chuang, H.-Y.; Kuo, C.C.; Chang, C.-Y.; Chang, J.-Y.; Liou, J.-P. Bioorg. Med. Chem. 2015, 23, 4230; (b) Patel, V. K.; Rajak, H. Bioorg. Med. Chem. Lett. 2016, 26, 2115 .

${ }^{4}$ Piatnitski Chekler, E. L.; Katoch-Rouse, R.; Kiselyov, A. S.; Sherman, D.; Ouyang, X.; Kim, K.; Wang, Y.; Hadari, Y. R.; Doody, J. F. Bioorg. Med. Chem. 2008, 18, 4344.

${ }^{5}$ Jetter, P.; Steinert, C.; Knauer, M.; Zhang, G.; Bruhn, T.; Wiese, J.; Imhoff, J. F.; Fiedler, H.-P.; Bringmann, G. J. Antibiot. 2013, 66, 719 .

${ }^{6}$ (a) Okauchi, T.; Itonaga, M.; Minami, T.; Owa, T.; Kitoh, K.; Yoshino, H. Org. Lett. 2000, 2, 1485; (b) Wynne, J. H.; Lloyd, C. T.; Jensen, S. D.; Boson, S.; Stalick, W. M. Synthesis 2004, 2277; (c) Yeung, K.-S.; Farkas, M. E.; Qiu, Z.; Yang, Z. Tetrahedron Lett. 2002, 43, 5793; (d) Guchhait, S. K.; Kashyap, M.; Kamble, H. J. Org. Chem. 2011, 76, 4753. 4649.

${ }^{7}$ Eyley, S. C.; Giles, R. G.; Heaney, H. Tetrahedron Lett. 1985, 26,

${ }^{8}$ Vekariya, R. H.; Aubé, J. Org. Lett. 2016, 18, 3534.

${ }_{9}^{9}$ (a) Bergman, J.; Venemalm, L. Tetrahedron Lett. 1987, 28, 3741; (b) Faul, M. M.; Winneroski, L. L. Tetrahedron Lett. 1997, 38, 4749.

${ }^{10}$ (a) Ma, Y.; You, J.; Song, F. Chem. Eur. J. 2013, 19, 1189; (b) Zhao, M.-N.; Ran, L.; Chen, M.; Ren, Z.-H.; Wang, Y.-Y.; Guan, Z.H. ACS Catal. 2015, 5, 1210; (c) Wang, Z.; Yin, Z.; Wu, X.-F. Org. Lett. 2017, 19, 4680

${ }^{11}$ Examples leading to the formation of pyrrole-derived ketones: (a) Fitton, A. O.; Frost, J. R.; Suschitzky, H.; Houghton, P. G. Synthesis 1977, 133; (b) Fitton, A. O.; Kosmirak, M.; Suschitzky, H.; Suschitzky, J. L. Tetrahedron Lett. 1982, 23, 3953; (c) Clarke, P. D.; Fitton, A. O.; Kosmirak, M.; Suschitzky, H.; Suschitzky, J. L. J. Chem. Soc., Perkin Trans. 1 1985, 1747; (d) Terzidis, M.; Tsoleridis, C. A.; Stephanidou-
Stephanatou, J. Tetrahedron 2007, 63, 7828; (e) Plaskon, A. S.; Ryabukhin, S. V.; Volochnyuk, D. M.; Shivanyuk, A. N.; Tolmachev, A. A. Tetrahedron 2008, 64, 5933; (f) Qi, X.; Xiang, H.; Yang, Y.; Yang, C. RSC Adv. 2015, 5, 98549.

${ }^{12}$ Single reported example of 3-salicyloyl indoles synthesis: Löwe, W.; Witzel, S.; Tappmeyer, S.; Albuschat, R. J. Heterocycl. Chem. 2004, 41, 317.

${ }^{13}$ Kim, Y.; Kim, J.; Park, S. B.; Org. Lett. 2009, 11, 17.

${ }^{14}$ Feng, C.-T.; Zhu, H.-Z.; Li, Z.; Luo, Z.; Wua, S.-S.; Ma, S.-T. Tetrahedron Lett. 2016, 57, 800.

${ }^{15}$ (a) Satoh, T.; Itaya, T.; Miura, M.; Nomura, M. Chem. Lett. 1996, 25, 823; (b) Park, Y. J.; Park, J.-W.; Jun, C.-H. Acc. Chem. Res. 2008, 41, 222; (c) Garralda, M. A. Dalton Trans. 2009, 3635; (d) Willis, M. C. Chem. Rev. 2010, 110, 725; (e) Ghosh, A.; Johnson, K. F.; Vickerman, K. L.; Walker, J. A. Jr.; Stanley, L. M. Org. Chem. Front. 2016, 3, 639;

${ }^{16}$ Yoshimura, A.; Zhdankin, V. V. Chem. Rev. 2016, 116, 3328.

17 (a) Chen, D.-J.; Chen, Z.-C. Synlett 2000, 8, 1175; (b) Ai, W.; Wu, Y.; Tang, H.; Yang, X.; Yang, Y.; Lib, Y.; Zhou, B. Chem. Commun. 2015, 51, 7871; (c) Wang, H.; Xie, F.; Qi, Z.; Li, X. Org. Lett. 2015, 17, 920; (d) Yang, X.; H. Wang, H.; Zhou, X.; Li, X. Org. Biomol. Chem. 2016, 14, 5233 .

${ }^{18}$ (a) Moriarty, R. M.; Ku, Y. Y.; Sultana, M.; Tuncay, A. Tetrahedron Lett. 1987, 28, 3071; (b) Moriyama, K.; Ishida, K.; Togo, H. Chem. Commun. 2015, 51, 2273; (c) Ishida, K.; Togo, H.; Moriyama, K. Chem. Asian J. 2016, 11, 3583; (d) Arun, V.; Venkataramana Reddy, P. O.; Pilania, M.; Kumar, D. Eur. J. Org. Chem. 2016, 12, 2096; (e) Liu, C.; Yi, J.-C.; Liang, X.-W.; Xu, R.-Q.; Dai, L.-X.; You, S.-L. Chem. Eur. J. 2016, 22, 10813;

${ }^{19}$ (a) Caramenti, P.; Nicolai, S.; Waser, J. Chem. Eur. J. 2017, 23, 14702; (b) Caramenti, P.; Waser, J. Helv. Chim. Acta, ASAP, DOI 10.1002/hlca.201700221; (c) Wu, B.; Wu, J.; Yoshikai, N. Chem. Asian J., 2017, 12, 3123..

${ }^{20}$ See Supporting Information for details.

${ }^{21}$ No desired product was observed with benzaldehyde, ortho-anisaldehyde or 2-acetoxybenzaldehyde as substrates. See Supporting Information.

${ }^{22}$ (a) Huffman, J. W.; Zengin, G.; Wu, M.-J.; Lu, J.; Hynd, G.; Bushell, K.; Thompson, A. L. S.; Bushell, S.; Tartal, C.; Hurst, D. P.; Reggio, P. H.; Selley, D. E.; Cassidy, M. P.; Wiley, J. L.; Martin, B. R. Bioorg. Med. Chem. 2005, 13, 89; (b) Nakajima, J.; Takahashi, M.; Nonaka, R.; Seto, T.; Suzuki, J.; Yoshida, M.; Kanai, C.; Hamano, T. Forensic Toxicol. 2011, 29, 132.

${ }^{23}$ For an example of iodine mediated $\mathrm{C} 2$ amination of indoles, see: Li, Y.-X.; Wang, H.-X.; Ali, S.; Xia, X.-F.; Liang, Y.-M. Chem. Commun. 2012, 48, 2343. In our work, this methodology is applied for the first time to the synthesis of quinolinones. 


\section{Supporting Information}

72 pages

\section{Iridium- and Rhodium-Catalyzed Directed C-H Heteroarylation of Benzaldehydes with Benziodoxolone Hypervalent Iodine Reagents}

Erwann Grenet and Jérôme Waser

Laboratory of Catalysis and Organic Synthesis, Institute of Chemical Sciences and Engineering, Ecole Polytechnique Fédérale de Lausanne, EPFL SB ISIC LCSO, BCH 4306, 1015 Lausanne, Switzerland.

jerome.waser@epfl.ch 


\section{Contents}

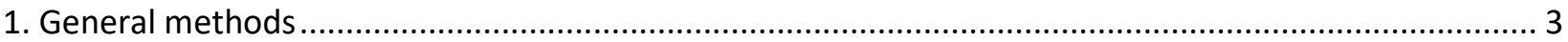

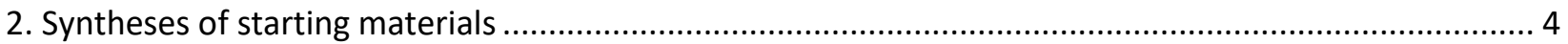

2. 1. General procedure $A$ for the synthesis of iridium and rhodium complexes ................................ 4

2.2. Procedures for the synthesis of 2 -hydroxybenzaldehydes ......................................................... 5

2.3. General procedure B for the synthesis of $N$-(2-formylphenyl)sulfonamides ............................... 6

2.4. General procedure $\mathrm{C}$ for the synthesis of $\mathrm{N}$-alkylindoles....................................................... 8

2.5. General procedure $D$ for the synthesis of indoleBX and pyrroleBX reagents .............................. 10

3. Optimization of the transition metal-catalyzed C-H activation........................................................ 13

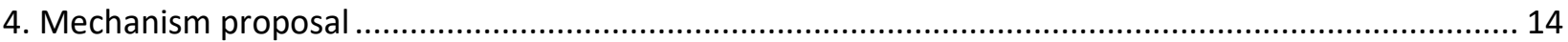

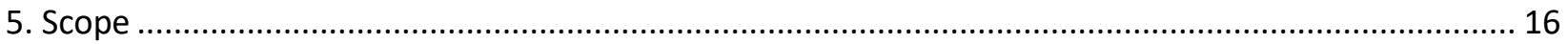

5.1. General procedure E1 for the synthesis of 3-salicyloylindoles .................................................... 16

5.2 General procedure E2 for the synthesis of 3-(2-sulfonamino)benzoylindoles ............................... 16

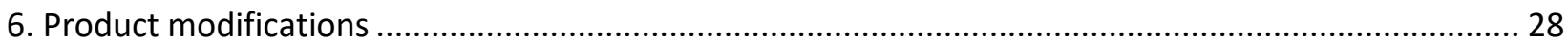

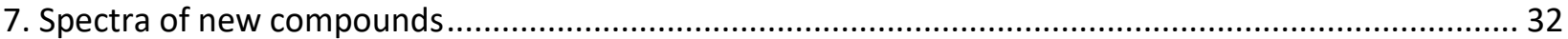




\section{General methods}

All reagents and solvents were purchased from commercial sources ABCR, Acros, Sigma Aldrich, Fluka, VWR, Aplichem or Merck and used as such unless stated otherwise. Chromatographic purification was performed as flash chromatography using Macherey-Nagel silica 40-63, 60 A. TLC was performed on Merck silica gel 60 F254 TLC glass plates and visualized with UV light, permanganate stain or phosphomolybdic acid stain. Melting points were measured on a calibrated Büchi B-540 melting point apparatus using open glass capillaries. ${ }^{1} \mathrm{H}-\mathrm{NMR}$ spectra were recorded on a Bruker DPX-400 $400 \mathrm{MHz}$ spectrometer in $\mathrm{CDCl}_{3}, \mathrm{CD}_{2} \mathrm{Cl}_{2}$, DMSO-d6, $\mathrm{CD}_{3} \mathrm{OD}$; all signals are reported in ppm with the internal chloroform signal at $7.26 \mathrm{ppm}$, the internal $\mathrm{CD} 2 \mathrm{Cl} 2$ signal at $5.32 \mathrm{ppm}$, the internal DMSO signal at 2.50 $\mathrm{ppm}$ or the internal methanol signal at $3.30 \mathrm{ppm}$ as standard. The data is being reported as $(\mathrm{s}=\operatorname{singlet} \mathrm{d}=$ doublet $\mathrm{t}=$ triplet $\mathrm{q}=$ quadruplet quint $=$ quintet $\mathrm{m}=$ multiplet or unresolved $\mathrm{bs}=$ broad signal, coupling constant(s) in Hz, integration, interpretation). 19F-NMR spectra were recorded on a Bruker DPX-400 376 $\mathrm{MHz}$ spectrometer in $\mathrm{CDCl}_{3} .{ }^{13} \mathrm{C}-\mathrm{NMR}$ spectra were recorded with $1 \mathrm{H}$-decoupling on a Bruker DPX-400 $101 \mathrm{MHz}$ spectrometer in $\mathrm{CDCl}_{3}, \mathrm{CD}_{2} \mathrm{Cl}_{2}$, DMSO-d6, $\mathrm{CD}_{3} \mathrm{OD}$; all signals are reported in ppm with the internal chloroform signal at $77.2 \mathrm{ppm}$, the internal $\mathrm{CD} 2 \mathrm{Cl} 2$ signal at $54.0 \mathrm{ppm}$, the internal DMSO signal at $39.5 \mathrm{ppm}$ or the internal methanol signal at $49.0 \mathrm{ppm}$ as standard. Infrared spectra were recorded on a JASCO FT-IR B4100 or a Brucer Alpha-P spectrophotometer with an ATR device and a ZnSe prisma and are reported $\mathrm{as}_{\mathrm{cm}}^{-1}(\mathrm{w}=$ weak, $\mathrm{m}=$ medium, $\mathrm{s}=$ strong, $\mathrm{br}=$ broad). High resolution mass spectrometric measurements were performed by the mass spectrometry service of ISIC at the EPFL on a MICROMASS (ESI) Q-TOF Ultima API. 


\section{Syntheses of starting materials}

\section{1. General procedure A for the synthesis of iridium and rhodium complexes}
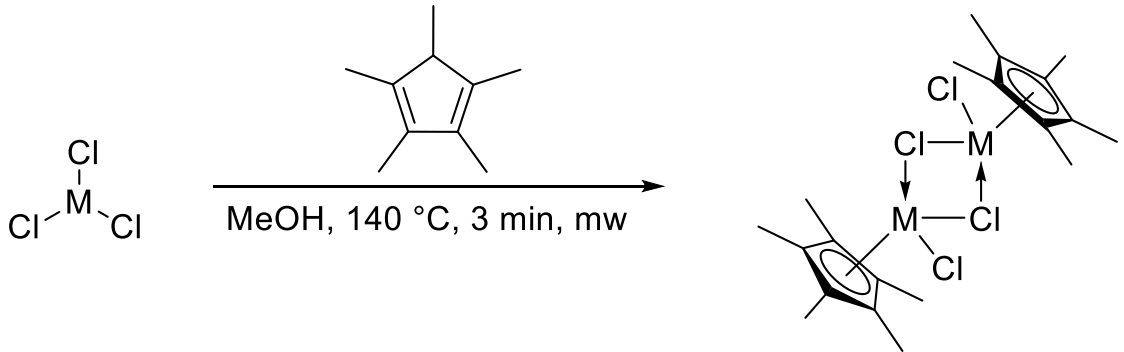

Following a reported procedure, ${ }^{1}$ the metal trichloride hydrate (1.00 equiv) and 1,2,3,4,5pentamethylcyclopenta-1,3-diene (1.50 equiv) were solubilized in $\mathrm{MeOH}[0.2 \mathrm{M}]$. The mixture was stirred at $140{ }^{\circ} \mathrm{C}$ for $3 \mathrm{~min}$ under microwave irradiation. The mixture was washed three times with pentane (same volume as $\mathrm{MeOH}$ ). $\mathrm{Et}_{2} \mathrm{O}$ (same volume as $\mathrm{MeOH}$ ) was added, the precipitate was isolated, washed twice with $\mathrm{Et}_{2} \mathrm{O}$ and dried under reduced pressure to afford the title compound.

\section{Pentamethylcyclopentadienyliridium (III) dichloride dimer [IRCp*Cl $\left.\mathrm{I}_{2}\right]_{2}$}

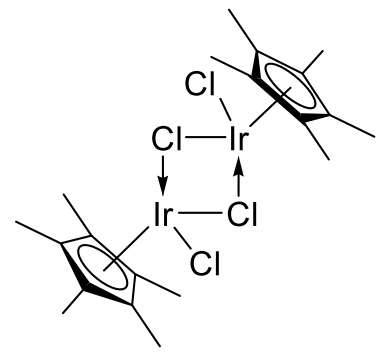

Following general procedure $\mathrm{A}$ and starting from commercially available iridium (III) chloride hydrate $(245 \mathrm{mg}, 0.820 \mathrm{mmol})$, pentamethylcyclopentadienyliridium (III) dichloride dimer (264 mg, $0.320 \mathrm{mmol}, 39 \%$ yield) (CAS number 12354-84-6) was obtained as an orange solid.

${ }^{1} \mathbf{H}$ NMR $\left(400 \mathrm{MHz}, \mathrm{CDCl}_{3}\right) \delta 1.62(\mathrm{~s}, 30 \mathrm{H}){ }^{2}$

\section{Pentamethylcyclopentadienylrhodium (III) chloride dimer $\left[\mathrm{RhCp} * \mathrm{Cl}_{2}\right]_{2}$}

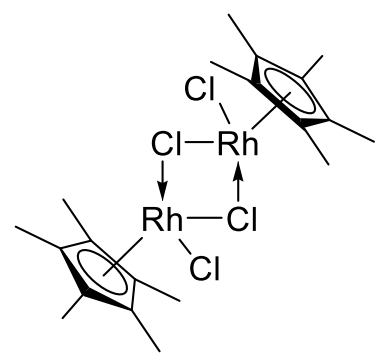

Following general procedure $\mathrm{A}$ and starting from commercially available rhodium (III) chloride hydrate $(385 \mathrm{mg}, 1.84 \mathrm{mmol})$, pentamethylcyclopentadienylrhodium (III) dichloride dimer $(382 \mathrm{mg}, 0.590 \mathrm{mmol}, 67 \%$ yield) (CAS number 12354-85-7) was obtained as a red solid.

${ }^{1} \mathbf{H}$ NMR $\left(400 \mathrm{MHz}, \mathrm{CDCl}_{3}\right) \delta 1.59(\mathrm{~s}, 30 \mathrm{H})$.

\footnotetext{
${ }^{1}$ Tönnemann, J.; Risse, J.; Grote, Z.; Scopelliti, R.; Severin, K. Eur. JIC 2013, 4558-4562.

2 Vázquez-Villa, H.; Reber, S.; Ariger, M. A.; Carreira, E. M. Angew. Chem. Int. Ed. 2011, 50 , 8979 -8981.
} 


\subsection{Procedures for the synthesis of 2-hydroxybenzaldehydes}

The reagents $\mathbf{4 a - k ,} \mathbf{4 m}, \mathbf{4 n}$ and $\mathbf{4 o}$ are commercially available.<smiles>COc1cccc(OC)c1C=O</smiles>

methoxybenzaldehyde (4I)

Aluminium trichloride $(2.00 \mathrm{~g}, 15.0 \mathrm{mmol}, 3.00$ equiv.) were suspended in dry DCM (20 $\mathrm{mL})$ under Ar. Commercially available 2,6-dimethoxybenzaldehyde ( $831 \mathrm{mg}, 5.00 \mathrm{mmol}$, 1.00 equiv) was solubilized in dry DCM $(20 \mathrm{~mL})$ under Ar and added to the solution at $\mathrm{rt}$. The mixture was stirred $24 \mathrm{~h}$ at $\mathrm{rt}$ and quenched with aqueous $1 \mathrm{M} \mathrm{HCl}$ solution (20 $\mathrm{mL}$ ). The two layers were separated and the aqueouse layer was extracted three times with DCM $(20 \mathrm{~mL})$. The organic layers were combined, dried over $\mathrm{MgSO}_{4}$ and concentrated under reduced pressure. The crude product was purified by column chromatography on silica gel (Pentane/EtOAc 4:1) affording the title compound $\mathbf{4 l}(210 \mathrm{mg}, 1.38 \mathrm{mmol}, 27 \%$ yield) as a white solid.

${ }^{1} \mathbf{H}$ NMR $\left(400 \mathrm{MHz}, \mathrm{CDCl}_{3}\right) \delta 11.98(\mathrm{~s}, 1 \mathrm{H}, \mathrm{OH}), 10.33(\mathrm{~s}, 1 \mathrm{H}, \mathrm{CHO}), 7.41\left(\mathrm{t}, J=8.4 \mathrm{~Hz}, 1 \mathrm{H}, \underline{\mathrm{H}}_{\mathrm{Ar}}\right), 6.52$ $\left(\mathrm{d}, J=8.5 \mathrm{~Hz}, 1 \mathrm{H}, \underline{\mathrm{H}}_{\mathrm{Ar}}\right), 6.37\left(\mathrm{~d}, J=8.3 \mathrm{~Hz}, 1 \mathrm{H}, \underline{\mathrm{H}}_{\mathrm{Ar}}\right), 3.89\left(\mathrm{~s}, 3 \mathrm{H}, \overline{\mathrm{OCH}}_{3}\right) .{ }^{13} \mathbf{C} \mathbf{N M R}\left(101 \mathrm{MHz}, \mathrm{CDCl}_{3}\right) \delta$ $194.5,163.8,162.6,138.6,111.0,110.0,101.1,56.0$. Spectra data matched with the values reported in literature. ${ }^{3}$

\section{$(8 R, 9 S, 13 S, 14 S)$-3-Hydroxy-13-methyl-17-oxo-7,8,9,11,12,13,14,15,16,17-decahydro-6H-cyclopenta-} [a]phenanthrene-2-carbaldehyde (4p)

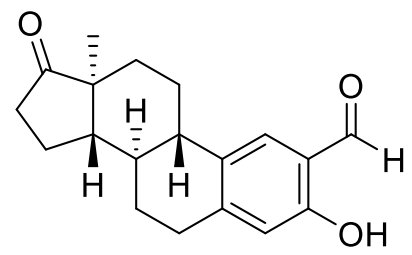

Following a reported procedure, ${ }^{4}$ commercially available estrone $(243 \mathrm{mg}$, $0.899 \mathrm{mmol}, 1.00$ equiv), magnesium chloride (257 $\mathrm{mg}, 2.70 \mathrm{mmol}, 3.00$ equiv) and formaldehyde $(81.0 \mathrm{mg}, 2.70 \mathrm{mmol}, 3.00$ equiv. $)$ were suspended in dry THF $(9 \mathrm{~mL})$ under Ar. Triethylamine $(0.50 \mathrm{~mL}, 3.6 \mathrm{mmol}, 4.0$ equiv.) was added to the solution and the mixture was stirred $2 \mathrm{~h}$ at reflux. The solution was quenched with aqueous $4 \mathrm{M} \mathrm{HCl}$ solution $(10 \mathrm{~mL})$ and extracted three times with EtOAc $(10 \mathrm{~mL})$. The organic layers were combined, dried over $\mathrm{MgSO}_{4}$ and concentrated under reduced pressure. The crude product was purified by column chromatography on silica gel (Pentane/EtOAc 4:1) affording the title compound $4 \mathbf{p}(180 \mathrm{mg}, 0.602 \mathrm{mmol}, 67 \%$ yield) as a white solid.

${ }^{1} \mathbf{H}$ NMR $\left(400 \mathrm{MHz}, \mathrm{CDCl}_{3}\right) \delta 10.77(\mathrm{~s}, 1 \mathrm{H}, \mathrm{OH}), 9.79(\mathrm{~s}, 1 \mathrm{H}, \mathrm{CHO}), 7.41\left(\mathrm{~s}, 1 \mathrm{H}, \underline{\mathrm{H}}_{\mathrm{Ar}}\right), 6.69\left(\mathrm{~s}, 1 \mathrm{H}, \underline{\mathrm{H}}_{\mathrm{Ar}}\right)$, 2.97-2.85 (m, $\left.2 \mathrm{H}, \underline{\mathrm{H}}_{\mathrm{aliph}}\right), 2.50\left(\mathrm{dd}, J=18.9,8.6 \mathrm{~Hz}, 1 \mathrm{H}, \underline{\mathrm{H}}_{\mathrm{aliph}}\right), 2.40\left(\mathrm{~s}, 1 \mathrm{H}, \underline{\mathrm{H}}_{\mathrm{aliph}}\right), 2.27-1.96$ (m, 5H, $\left.\underline{\mathrm{H}}_{\mathrm{aliph}}\right)$, $1.67-1.38\left(\mathrm{~m}, 6 \mathrm{H}, \underline{\mathrm{H}}_{\text {aliph }}\right), 0.91\left(\mathrm{~s}, 3 \mathrm{H}, \mathrm{CH}_{3}\right) .{ }^{13} \mathbf{C} \mathbf{N M R}\left(101 \mathrm{MHz}, \mathrm{CDCl}_{3}\right) \delta 196.2,159.4,147.8,132.2$, 130.6, 119.1, 117.1, 50.5, 48.0, 43.5, 38.0, 35.9, 31.5, 30.1, 26.2, 25.9, 21.7, 13.9. Spectra data matched with the values reported in literature. ${ }^{4}$

\section{4-Hydroxy-9H-carbazole-3-carbaldehyde (4q)}

Following a reported procedure, ${ }^{5}$ phosphoryl trichloride $(0.400 \mathrm{~mL}, 4.30 \mathrm{mmol}, 1.20$ equiv.) was slowly<smiles>O=Cc1ccc2[nH]c3ccccc3c2c1O</smiles>
added to DMF (2 mL) at $15{ }^{\circ} \mathrm{C}$ under Ar. $9 H$-carbazol-4-ol $(655 \mathrm{mg}, 3.58 \mathrm{mmol}$, 1.00 equiv.) was solubilized in DMF (4 mL) and slowly added at rt. The mixture was stirred $1 \mathrm{~h}$ at $35{ }^{\circ} \mathrm{C}$ and poured into crush ice and treated with $1 \mathrm{M} \mathrm{NaOH}(5$ $\mathrm{mL})$. The mixture was extracted three times with EtOAc $(10 \mathrm{~mL})$. The organic layers were combined, dried over $\mathrm{MgSO}_{4}$ and concentrated under reduced pressure. The crude product was purified by column chromatography on silica gel (Pentane/EtOAc 7:3) and washed with $\mathrm{Et}_{2} \mathrm{O}$ affording the title compound 4q (430 mg, $2.04 \mathrm{mmol}, 57 \%$ yield) as a grey solid.

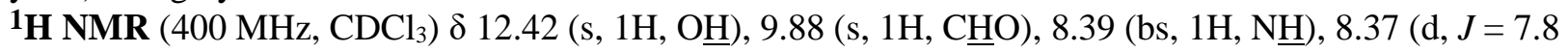
$\left.\mathrm{Hz}, 1 \mathrm{H}, \underline{\mathrm{H}}_{\mathrm{Ar}}\right), 7.50\left(\mathrm{~d}, J=8.4 \mathrm{~Hz}, 1 \mathrm{H}, \underline{\mathrm{H}}_{\mathrm{Ar}}\right), 7.4 \overline{5}\left(\mathrm{~d}, J=3.9 \mathrm{~Hz}, 2 \overline{\mathrm{H}}, \underline{\mathrm{H}}_{\mathrm{Ar}}\right), 7.35\left(\mathrm{~m}, 1 \mathrm{H}, \underline{\mathrm{H}}_{\mathrm{Ar}}\right), 7.02(\mathrm{~d}, J=$ $\left.8.4 \mathrm{~Hz}, 1 \mathrm{H}, \underline{\mathrm{H}}_{\mathrm{Ar}}\right) .{ }^{13} \mathrm{C}$ NMR $\left(101 \mathrm{MHz}, \mathrm{CDCl}_{3}\right) \delta 195.2,160.4,145.4,138.8,131.5,126.0,123.4,122.9$, $121.5,113.7,111.5,110.7,103.6$. Spectra data differs slightly from the values reported in literature. ${ }^{5}$

\footnotetext{
${ }^{3}$ Yoshioka, E.; Kohtani, S.; Miyabe, H. Org. Lett. 2010, 12, 1956-1959.

${ }^{4}$ Akselsen, Ø. W.; Hansen, T. V. Tetrahedron 2011, 67, 7738-7742.

${ }^{5}$ Bhosale, S. M.; Momin, A. A.; Kusurkar, R. S. Tetrahedron 2012, 68, 6420-6426.
} 


\subsection{General procedure B for the synthesis of $N$-(2-formylphenyl)sulfonamides}<smiles>Nc1ccccc1CO</smiles><smiles></smiles><smiles>[R4]S(=O)(=O)Nc1ccccc1CO</smiles><smiles></smiles><smiles>[R]S(=O)(=O)Nc1ccccc1C=O</smiles>

(2-aminophenyl)methanol (1.00 equiv) and the corresponding sulfonyl chloride (1.10 equiv) were solubilized in $\mathrm{CHCl}_{3}[0.4 \mathrm{M}]$. Pyridine (1.20 equiv) was added to the solution and the mixture was stirred overnight at room temperature. The mixture was concentrated under reduced pressure. The residue was taken up in EtOAc $(30 \mathrm{~mL})$ and washed three times with an aqueous $4 \mathrm{M} \mathrm{HCl}$ solution $(15 \mathrm{~mL})$ and once with a $\mathrm{NaHCO}_{3}$ solution $(15 \mathrm{~mL})$. The organic layer was dried over magnesium sulfate dehydrate, filtered and concentrated under reduced pressure. The desired $N$-(2-(hydroxymethyl)phenyl)sulfonamide, obtained without further purification, was solubilized in DCM [0.3 M]. Pyridinium chlorochromate (1.50 equiv) was added in one portion and the mixture was stirred at room temperature for 2 hours. The solution was filtered on a $5 \mathrm{~cm}$ pad of silica gel then washed with DCM $(100 \mathrm{~mL})$. The filtrate was concentrated under reduced pressure. The residue was purified by recrystallization in $\mathrm{EtOH} / \mathrm{CHCl}_{3} 4: 1$ to afford the title compound.

\section{$N$-(2-Formylphenyl)4-methylbenzenesulfonamide (7a)}<smiles>Cc1ccc(S(=O)(=O)Nc2ccccc2C=O)cc1</smiles>

Following general procedure $\mathbf{B}$ and starting from commercially available (2aminophenyl)methanol $\quad(0.75 \quad \mathrm{~g}, \quad 6.1 \quad \mathrm{mmol}), \quad N$-(2-formylphenyl)4methylbenzenesulfonamide 7a (1.40 g, $5.08 \mathrm{mmol}, 83 \%$ yield) (CAS number 659065-4) was obtained as a white solid.

${ }^{1} \mathbf{H}$ NMR $\left(400 \mathrm{MHz}, \mathrm{CDCl}_{3}\right) \delta 10.79$ (s, 1H, NHTs), 9.83 (s, 1H, CHO), 7.77 (d, J = $\left.8.3 \mathrm{~Hz}, 2 \mathrm{H}, \underline{\mathrm{H}}_{\mathrm{tolyl}}\right), 7.69\left(\mathrm{~d}, \mathrm{~J}=8.4 \mathrm{~Hz}, 1 \mathrm{H}, \underline{\mathrm{H}}_{\mathrm{Ar}}\right), 7.59\left(\mathrm{dd}, \mathrm{J}=7.7,1.5 \mathrm{~Hz}, 1 \mathrm{H}, \underline{\mathrm{H}}_{\mathrm{Ar}}\right)$, $7.51\left(\mathrm{td}, \mathrm{J}=8.6,8.1,1.6 \mathrm{~Hz}, 1 \mathrm{H}, \underline{\mathrm{H}}_{\mathrm{Ar}}\right), 7.24\left(\mathrm{~d}, \mathrm{~J}=8.0 \mathrm{~Hz}, 2 \mathrm{H}, \underline{\mathrm{H}}_{\mathrm{tolyl}}\right), 7.16(\mathrm{td}, \mathrm{J}=$ 7.6, $\left.0.9 \mathrm{~Hz}, 1 \mathrm{H}, \underline{\mathrm{H}}_{\mathrm{Ar}}\right), 2.37\left(\mathrm{~s}, 3 \mathrm{H}, \underline{\mathrm{C}}_{3}\right)$.

${ }^{13}$ C NMR $\left(101 \mathrm{MHz}, \mathrm{CDCl}_{3}\right) \delta 195.1,144.3,140.1,136.5,136.2,136.0,129.9,127.4,123.1,122.0,117.9$, 21.7. Spectra data matched with the values reported in literature. ${ }^{6}$

\section{$N$-(2-Formylphenyl)4-nitrobenzenesulfonamide (7b)}<smiles>O=Cc1ccccc1NS(=O)(=O)c1ccc([N+](=O)[O-])cc1</smiles>

$\left.0.8 \mathrm{~Hz}, 1 \mathrm{H}, \underline{\mathrm{H}}_{\mathrm{Ar}}\right)$.

Following general procedure $\mathbf{B}$ and starting from commercially available (2aminophenyl)methanol $\quad(0.75 \quad \mathrm{~g}, \quad 6.1 \mathrm{mmol}), \quad \mathrm{N}$-(2-formylphenyl)4nitrobenzenesulfonamide 7b (1.20 g, $3.92 \mathrm{mmol}$, 64\% yield) (CAS number 601481-97-4) was obtained as a yellow solid.

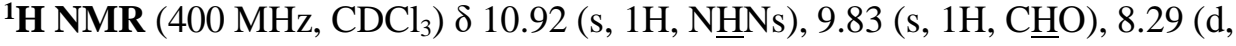
$\left.J=8.9 \mathrm{~Hz}, 2 \mathrm{H}, \underline{\mathrm{H}}_{\text {nosyl }}\right), 8.06\left(\mathrm{~d}, J=8.9 \mathrm{~Hz}, 2 \mathrm{H}, \underline{\mathrm{H}}_{\text {nosyl }}\right), 7.73(\mathrm{~d}, J=8.4 \mathrm{~Hz}, 1 \mathrm{H}$, $\left.\underline{\mathrm{H}}_{\mathrm{Ar}}\right), 7.64\left(\mathrm{dd}, J=7.6,1.5 \mathrm{~Hz}, 1 \mathrm{H}, \underline{\mathrm{H}}_{\mathrm{Ar}}\right), 7.59-7.55\left(\mathrm{~m}, 1 \mathrm{H}, \underline{\mathrm{H}}_{\mathrm{Ar}}\right), 7.25(\mathrm{td}, J=7.6$,

${ }^{13} \mathrm{C}$ NMR (101 MHz, $\left.\mathrm{CDCl}_{3}\right) \delta$ 195.3, 150.5, 145.1, 139.0, 136.5, 136.2, 128.7, 124.6, 124.2, 122.4, 118.3.

${ }^{1} \mathrm{H}$ NMR Spectra data matched with the values reported in literature. ${ }^{5}$

\footnotetext{
${ }^{6}$ Hechavarría Fonseca, M.; Eibler, E.; Zabelb, M.; König, B. Tetrahedron: Asymmetry 2003, 14, 1989-1994.
} 
<smiles>CS(=O)(=O)Nc1ccccc1C=O</smiles>

Following general procedure $\mathbf{B}$ and starting from commercially available (2aminophenyl)methanol (0.75 g, $6.1 \mathrm{mmol}), N$-(2-formylphenyl)methanesulfonamide 7c (0.680 g, $3.42 \mathrm{mmol}, 56 \%$ yield) (CAS number 94532-99-7) was obtained as a white solid.

${ }^{1} \mathbf{H}$ NMR $\left(400 \mathrm{MHz}, \mathrm{CDCl}_{3}\right) \delta 10.60$ (s, $\left.1 \mathrm{H}, \mathrm{N} \underline{\mathrm{HMs}}\right), 9.92$ (s, $\left.1 \mathrm{H}, \mathrm{C} \underline{\mathrm{HO}}\right), 7.73(\mathrm{t}, J=7.6 \mathrm{~Hz}$, $\left.2 \mathrm{H}, \underline{\mathrm{H}}_{\mathrm{Ar}}\right), 7.62\left(\mathrm{t}, J=7.9 \mathrm{~Hz}, 1 \mathrm{H}, \underline{\mathrm{H}}_{\mathrm{Ar}}\right), 7.28-7.24\left(\mathrm{~m}, 1 \mathrm{H}, \underline{\mathrm{H}}_{\mathrm{Ar}}\right), 3.10\left(\mathrm{~s}, 3 \mathrm{H}, \mathrm{C}_{3}\right) .{ }^{13} \mathbf{C ~ N M R}$ $\left(101 \mathrm{MHz}, \mathrm{CDCl}_{3}\right) \delta 195.2,140.3,136.6,136.3,123.1,121.7,117.1,40.5$. Spectra data matched with the values reported in literature. ${ }^{7}$

\section{$N$-(4-Chloro-2-formylphenyl)-4-methylbenzenesulfonamide (7d)}<smiles>Cc1ccc(S(=O)(=O)Nc2ccc(Cl)cc2C=O)cc1</smiles>

Following general procedure $\mathbf{B}$ and starting from commercially available (2amino-5-chlorophenyl)methanol (236 $\mathrm{mg}, \quad 1.50 \mathrm{mmol}), \quad \mathrm{N}$-(4-chloro-2formylphenyl)4-methylbenzenesulfonamide 7d (416 mg, $1.34 \mathrm{mmol}, 89 \%$ yield) (CAS number 34159-03-0) was obtained as a white solid.

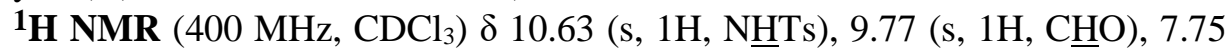
$\left(\mathrm{d}, \mathrm{J}=8.3 \mathrm{~Hz}, 2 \mathrm{H}, \underline{\mathrm{H}}_{\text {tolyl }}\right), 7.68\left(\mathrm{~d}, \mathrm{~J}=8.9 \mathrm{~Hz}, 1 \mathrm{H}, \underline{\mathrm{H}}_{\mathrm{Ar}}\right), 7.55(\mathrm{~d}, \mathrm{~J}=2.5 \mathrm{~Hz}, 1 \mathrm{H}$, $\left.\underline{\mathrm{H}}_{\mathrm{Ar}}\right), 7.46\left(\mathrm{dd}, \mathrm{J}=8.9,2.5 \mathrm{~Hz}, 1 \mathrm{H}, \underline{\mathrm{H}}_{\mathrm{Ar}}\right), 7.25\left(\mathrm{~d}, \mathrm{~J}=8.3 \mathrm{~Hz}, 2 \mathrm{H}, \underline{\mathrm{H}}_{\mathrm{tolyl}}\right), 2.37(\mathrm{~s}$, $\left.3 \mathrm{H}, \mathrm{C}_{3}\right) .{ }^{13} \mathrm{C}$ NMR $\left(101 \mathrm{MHz}, \mathrm{CDCl}_{3}\right) \delta 193.9,144.6,138.6,136.2,135.8,135.3,130.0,128.5,127.4$, $122.9,119.7,21.7$. Spectra data matched with the values reported in literature. ${ }^{8}$

\section{$N$-(2-Formylthiophen-3-yl)-4-methylbenzenesulfonamide (7e)}

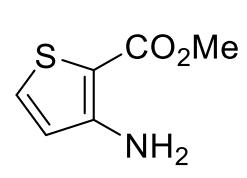

1) $\mathrm{TsCl}$, pyridine, $\mathrm{CHCl}_{3}$, rt, overnight

2) DIBAL-H, THF, $-78^{\circ} \mathrm{C}$ to $0^{\circ} \mathrm{C}, 4 \mathrm{~h}$

3) PCC, DCM, rt, $2 \mathrm{~h}$

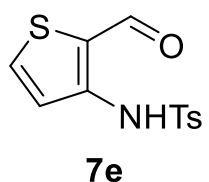

Methyl 3-amino-2-thiophenecarboxylate (1.00 equiv) and tosyl chloride (1.10 equiv) were solubilized in $\mathrm{CHCl}_{3}[0.4 \mathrm{M}]$. Pyridine (1.20 equiv) was added to the solution and the mixture was stirred overnight at room temperature. The mixture was concentrated under reduced pressure. The residue was taken up in EtOAc $(30 \mathrm{~mL})$ and washed three times with an aqueous $4 \mathrm{M} \mathrm{HCl}$ solution $(15 \mathrm{~mL})$ and once with a $\mathrm{NaHCO}_{3}$ solution $(15 \mathrm{~mL})$. The organic layer was dried over magnesium sulfate dehydrate, filtered and concentrated under reduced pressure. In a flame-dried flask, the methyl 3-(4-methylphenylsulfonamido)thiophene-2carboxylate, obtained without further purification, was solubilized in dry THF [0.2 M] under Ar. DIBAL-H (4.00 equiv.) was added to the solution at $-78{ }^{\circ} \mathrm{C}$ and the mixture was stirred $4 \mathrm{~h}$ until $0{ }^{\circ} \mathrm{C}$. The mixture was quenched with a saturated aqueous solution of Rochelle's salt $(10 \mathrm{~mL})$ and filtered over Celite. The layers were separated and the aqueous layer was extracted three times with EtOAc $(20 \mathrm{~mL})$. The organic layer was dried over magnesium sulfate dehydrate, filtered and concentrated under reduced pressure. The $N$-(2-(hydroxymethyl)thiophen-3-yl)-4-methylbenzenesulfonamide, obtained without further purification, was solubilized in dry DCM [0.2 M]. Pyridinium chlorochromate (1.50 equiv) was added in one portion and the mixture was stirred at room temperature for 2 hours. The solution was filtered on a $5 \mathrm{~cm}$ pad of silica gel then washed with DCM $(100 \mathrm{~mL})$. The filtrate was concentrated under reduced pressure to afford the title compound 7e (60\% yield over 3 steps). ${ }^{\mathbf{1}} \mathbf{H}$ NMR (400 MHz, $\left.\mathrm{CDCl}_{3}\right) \delta 10.14$ (s, 1H, NHTs), 9.59 (s, $1 \mathrm{H}, \mathrm{C} \underline{\mathrm{HO}}), 7.76\left(\mathrm{~d}, J=6.5 \mathrm{~Hz}, 2 \mathrm{H}, \underline{\mathrm{H}}_{\text {tolyl }}\right), 7.65-7.58\left(\mathrm{~m}, 1 \mathrm{H}, \underline{\mathrm{H}}_{\text {thiophenyl }}\right), 7.47-7.38\left(\mathrm{~m}, 1 \mathrm{H}, \underline{\mathrm{H}}_{\text {thiophenyl }}\right), 7.27$ $\left(\mathrm{d}, J=5.7 \mathrm{~Hz}, 2 \mathrm{H}, \underline{\mathrm{H}}_{\text {tolyl }}\right), 2.39$ (s, 3H, $\left.\underline{\mathrm{CH}}_{3}\right) .{ }^{13} \mathrm{C} \mathbf{N M R}\left(101 \mathrm{MHz}, \mathrm{CDCl}_{3}\right) \delta 184.0,144.6,143.8,136.4$, $136.4,130.0,127.2,121.0,120.7,21.7$. Spectra data matched with the values reported in literature. ${ }^{9}$

\footnotetext{
${ }^{7}$ Nemoto, T.; Fukuda, T.; Hamada, Y. Tetrahedron Letters 2006, 47, 4365-4368.

${ }^{8}$ Theeraladanon, C.; Arisawa, M.; Nishida, A.; Nakagawa, M. Tetrahedron 2004, 60, 3017-3035.

${ }^{9}$ Mu, D.; Wang, X.; Chen, G.; He, G. J. Org. Chem. 2017, 82, 4497-4503.
} 


\subsection{General procedure $\mathrm{C}$ for the synthesis of $N$-alkylindoles}
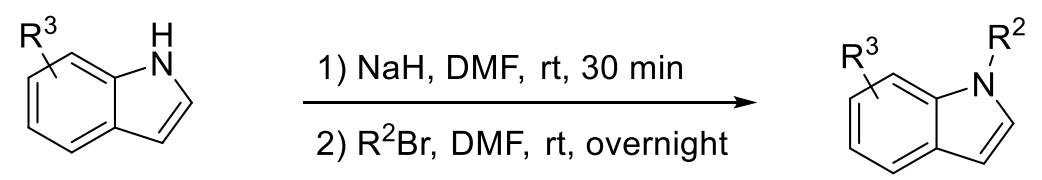

Sodium hydride (60\% in mineral oil, 1.10 equiv) was suspended in DMF [0.6 M]. $N$-H-indole (1.00 equiv) was solubilized in DMF [1.0 M] and added to the suspension at $0{ }^{\circ} \mathrm{C}$. The mixture was stirred at $\mathrm{rt}$ for 30 min. Bromoalkyl or TMSCl (1.50 equiv) was diluted in DMF $[3.0 \mathrm{M}]$ and added to the solution at $0{ }^{\circ} \mathrm{C}$. The mixture was stirred at $\mathrm{rt}$ for 1 hour. The solution was quenched with water $(20 \mathrm{~mL})$ and extracted three times with EtOAc $(10 \mathrm{~mL})$. The organic layers were combined, dried over $\mathrm{MgSO}_{4}$ and concentrated under reduced pressure. The liquid was filtered through a $5 \mathrm{~cm}$ pad of silica with $100 \%$ pentane or $\mathrm{Et}_{2} \mathrm{O}$ to afford the title compound.

\section{1-Pentyl-1H-indole (15b)}

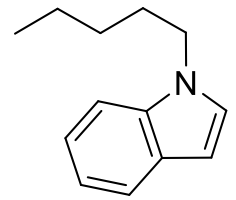

Following general procedure $\mathbf{C}$ and starting from commercially available indole $(1.32 \mathrm{~g}$, $11.3 \mathrm{mmol}$ ), $\mathbf{1 5 b}$ (2.11 g, $11.3 \mathrm{mmol}$, quantitative yield) (CAS number 59529-21-4) was obtained as a colorless liquid.

${ }^{1} \mathbf{H}$ NMR $\left(400 \mathrm{MHz}, \mathrm{CDCl}_{3}\right) \delta 7.66\left(\mathrm{~d}, J=7.9 \mathrm{~Hz}, 1 \mathrm{H}, \underline{\mathrm{H}}_{\mathrm{Ar}}\right), 7.37\left(\mathrm{~d}, J=8.2 \mathrm{~Hz}, 1 \mathrm{H}, \underline{\mathrm{H}}_{\mathrm{Ar}}\right)$, $7.23\left(\mathrm{t}, J=7.6 \mathrm{~Hz}, 1 \mathrm{H}, \underline{\mathrm{H}}_{\mathrm{Ar}}\right), 7.14-7.10\left(\mathrm{~m}, 2 \mathrm{H}, \underline{\mathrm{H}}_{\mathrm{Ar}}\right), 6.51\left(\mathrm{~d}, J=3.0 \mathrm{~Hz}, 1 \mathrm{H}, \underline{\mathrm{H}}_{\mathrm{Ar}}\right), 4.13(\mathrm{t}$, $\left.J=7.2 \mathrm{~Hz}, 2 \mathrm{H}, \mathrm{NC}_{2}\right), 1.86$ (quint, $\left.J=7.2 \mathrm{~Hz}, 2 \mathrm{H}, \underline{\mathrm{H}}_{\text {aliph }}\right), 1.42-1.20$ (m, 4H, $\underline{\mathrm{H}}_{\text {aliph }}$ ), 0.91 (t, $J=6.9 \mathrm{~Hz}, 3 \mathrm{H}$, $\mathrm{CH}_{3}$ ).

${ }^{13} \mathbf{C}$ NMR $\left(101 \mathrm{MHz}, \mathrm{CDCl}_{3}\right) \delta 136.1,128.7,127.9,121.4,121.0,119.3,109.5,100.9,46.5,30.1,29.3$, $22.5,14.1$. Spectra data matched with the values reported in literature. ${ }^{10}$

\section{1-(But-3-en-1-yl)-1H-indole (15c)}

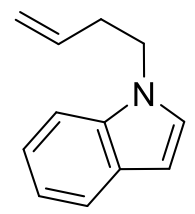

Following general procedure $\mathbf{C}$ and starting from commercially available indole (1.54 g, 13.1 $\mathrm{mmol}), 15 \mathrm{c}(0.39 \mathrm{~g}, 2.3 \mathrm{mmol}, 17 \%)$ (CAS number 46169-71-5) was obtained as a colorless liquid.

${ }^{1} \mathbf{H}$ NMR $\left(400 \mathrm{MHz}, \mathrm{CDCl}_{3}\right) \delta 7.64\left(\mathrm{~d}, J=7.9 \mathrm{~Hz}, 1 \mathrm{H}, \underline{\mathrm{H}}_{\mathrm{Ar}}\right), 7.36\left(\mathrm{~d}, J=8.2 \mathrm{~Hz}, 1 \mathrm{H}, \underline{\mathrm{H}}_{\mathrm{Ar}}\right)$, 7.24-7.20 (m, $\left.1 \mathrm{H}, \underline{\mathrm{H}}_{\mathrm{Ar}}\right), 7.13-7.09\left(\mathrm{~m}, 2 \mathrm{H}, \underline{\mathrm{H}}_{\mathrm{Ar}}\right), 6.49\left(\mathrm{~d}, J=3.1 \mathrm{~Hz}, 1 \mathrm{H}, \underline{\mathrm{H}}_{\mathrm{Ar}}\right), 5.80(\mathrm{ddt}, J=$ $\left.17.1,10.2,6.9 \mathrm{~Hz}, 1 \mathrm{H}, \mathrm{C} \underline{\mathrm{H}}=\mathrm{CH}_{2}\right), 5.11-5.05\left(\mathrm{~m}, 2 \mathrm{H}, \mathrm{CH}=\mathrm{C}_{2}\right), 4.20\left(\mathrm{t}, J=7.2 \mathrm{~Hz}, 2 \mathrm{H}, \mathrm{NC}_{2}\right), 2.59$ (q, $J$ $\left.=6.9 \mathrm{~Hz}, 2 \mathrm{H}, \mathrm{NCH}_{2} \mathrm{CH}_{2}\right)$.

${ }^{13}$ C NMR (101 MHz, $\left.\mathrm{CDCl}_{3}\right) \delta 136.0,134.8,128.7,127.9,121.5,121.1,119.4,117.5,109.5,101.2,46.1$, 34.7. Spectra data matched with the values reported in literature. ${ }^{11}$

\section{5-Bromo-1-methyl-1H-indole (15d)}

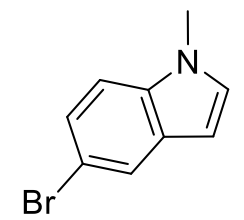

Following general procedure $\mathbf{C}$ and starting from commercially available 5-bromoindole $(0.59 \mathrm{mg}, 3.0 \mathrm{mmol}), \mathbf{1 5 d}(630 \mathrm{mg}, 3.00 \mathrm{mmol}$, quantitative yield) (CAS number 1007552-2) was obtained as a pale yellow solid.

${ }^{1} \mathbf{H}$ NMR $\left(400 \mathrm{MHz}, \mathrm{CDCl}_{3}\right) \delta 7.75\left(\mathrm{~d}, J=1.6 \mathrm{~Hz}, 1 \mathrm{H}, \underline{\mathrm{H}}_{\mathrm{Ar}}\right), 7.30(\mathrm{dd}, J=8.7,1.8 \mathrm{~Hz}, 1 \mathrm{H}$, $\left.\underline{\mathrm{H}}_{\mathrm{Ar}}\right), 3.78(\mathrm{~s}, 3 \mathrm{H}, \mathrm{NC})$.

${ }^{13}$ C NMR $\left(101 \mathrm{MHz}, \mathrm{CDCl}_{3}\right) \delta 135.5,130.2,130.1,124.4,123.4,112.8,110.8,100.6,33.1$.

Spectra data matched with the values reported in literature. ${ }^{12}$

\footnotetext{
${ }^{10}$ Banister, S. D.; Wilkinson, S. M.; Longworth, M.; Stuart, J.; Apetz, N.; English, K.; Brooker, L.; Goebel, C.; Hibbs, D. E.; Glass, M.; Connor, M.; McGregor, I. S.; Kassiou, M. ACS Chem. Neurosci. 2013, 4, 1081-1092.

${ }^{11}$ Kerchner, H. A.; Montgomery, J. Org. Lett. 2016, 18, 5760-5763.

12 Greulich, T.W.; Daniliuc, C.J.; Studer, A. Org. Lett. 2015, 17, 254-257.
} 


\section{1-(Tert-butyldimethylsilyl)-1H-indole (15e)}

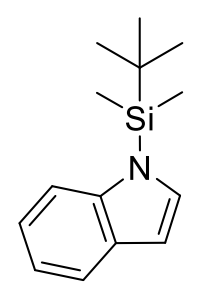

Following general procedure $\mathbf{C}$ and starting from commercially available indole ( $2.34 \mathrm{~g}, 20.0$ mmol), 15e (4.63 g, 20.0 mmol, quantitative yield) (CAS number 40899-73-8) was obtained as a pale yellow solid.

${ }^{1} \mathbf{H}$ NMR $\left(400 \mathrm{MHz}, \mathrm{CDCl}_{3}\right) \delta 7.56\left(\mathrm{~d}, J=7.2 \mathrm{~Hz}, 1 \mathrm{H}, \underline{\mathrm{H}}_{\mathrm{Ar}}\right), 7.44\left(\mathrm{~d}, J=7.7 \mathrm{~Hz}, 1 \mathrm{H}, \underline{\mathrm{H}}_{\mathrm{Ar}}\right)$, $7.10\left(\mathrm{~d}, J=3.2 \mathrm{~Hz}, 1 \mathrm{H}, \underline{\mathrm{H}}_{\mathrm{Ar}}\right), 7.09-6.99\left(\mathrm{~m}, 2 \mathrm{H}, \underline{\mathrm{H}}_{\mathrm{Ar}}\right), 6.54\left(\mathrm{~d}, J=2.7 \mathrm{~Hz}, 1 \mathrm{H}, \underline{\mathrm{H}}_{\mathrm{Ar}}\right), 0.85(\mathrm{~s}$, $\left.9 \mathrm{H}, \mathrm{C}\left(\mathrm{C}_{3}\right)_{3}\right), 0.52$ (s, 6H, Si $\left.\left(\mathrm{C}_{3}\right)_{2}\right)$.

${ }^{13} \mathbf{C}$ NMR $\left(101 \mathrm{MHz}, \mathrm{CDCl}_{3}\right) \delta 141.1,131.5,131.1,121.5,120.8,119.9,114.0,104.9,26.5$,

$19.7,-3.8$. Spectra data matched with the values reported in literature. ${ }^{13}$

${ }^{13}$ Dhanak, D.; Reese, C. B. J. Chem. Soc., Perkin Trans. 1 1986, 2181-2186 


\title{
2.5. General procedure $D$ for the synthesis of indoleBX and pyrroleBX reagents
}

\section{1-Acetoxy-1,2-benziodoxol-3-(1H)-one (16)}<smiles>O=C(O)c1ccccc1I</smiles>

\author{
$\underset{\mathrm{NaIO}_{4} \text {, aq. } 30 \% \mathrm{AcOH}}{100{ }^{\circ} \mathrm{C}, 4 \mathrm{~h}}$
}<smiles>O=C1OI(O)c2ccccc21</smiles>

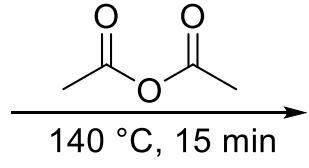<smiles>CC(=O)OI1C(=O)c2ccccc21</smiles>

Following a reported procedure ${ }^{14}$, sodium periodate $(18.1 \mathrm{~g}, 85.0 \mathrm{mmol}, 1.05$ equiv) and 2 -iodobenzoic acid (20.0 g, $81.0 \mathrm{mmol}, 1.00$ equiv) were suspended in $30 \%$ (v:v) aq. AcOH (160 mL). The mixture was vigorously stirred and refluxed for $4 \mathrm{~h}$ and allowed to cool to room temperature, while protecting it from light. After $1 \mathrm{~h}$, the crude product was collected by filtration. The crystals were washed with ice water $(3 \mathrm{x}$ $40 \mathrm{~mL}$ ) followed by acetone $(45 \mathrm{~mL})$ and dried under reduced pressure in the dark to afford 1-hydroxy-1,2benziodoxol-3-(1H)-one $(20.8 \mathrm{~g}, 79.0 \mathrm{mmol}, 98 \%)$ as a white solid. Following a reported procedure, 1hydroxy-1,2-benziodoxol-3-( $1 \mathrm{H})$-one (20.8 g, $79.0 \mathrm{mmol}, 1.00$ equiv.) was suspended in acetic anhydride ( $75.0 \mathrm{~mL}, 788 \mathrm{mmol}, 10.0$ equiv.) and heated to reflux $\left(140{ }^{\circ} \mathrm{C}\right)$ until complete dissolution (about $\left.15 \mathrm{~min}\right)$. The resulting clears solution was slowly let to cool to room temperature and then cooled to $5{ }^{\circ} \mathrm{C}$ in the fridge. The white crystals were filtered, washed with pentane $(3 \times 30 \mathrm{~mL})$ and dried under reduced pressure to afford 1-acetoxy-1,2-benziodoxol-3-(1H)-one (22.3 g, $73.0 \mathrm{mmol}, 92 \%)$ as a white solid.<smiles>CC(=O)OI1C(=O)OC1=O</smiles>

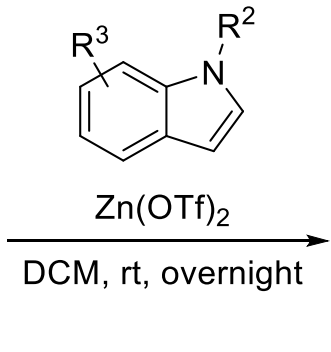

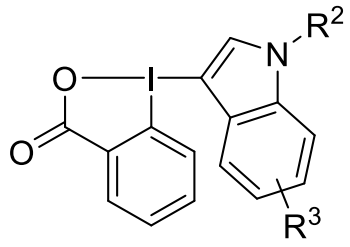

Following a slightly modified reported procedure ${ }^{15}$ 1-acetoxy-1,2- benziodoxol-3-(1H)-one (1.20 equiv), the corresponding azaheterocycle (1.00 equiv) and zinc (II) trifluoromethanesulfonate ( 0.20 equiv) were dissolved in DCM [0.05 M]. The reaction was stirred overnight at room temperature, directly purified by flash chromatography (eluent $\mathrm{DCM} / \mathrm{MeOH}$ see ratio thereafter) and triturated in $\mathrm{ACN}$ to afford the pure desired hypervalent iodine reagent.

\section{1-(3-1-Methyl-1H-indole)-1H-1 $\lambda_{3}$-benzo[b]iodo-3(2H)-one (5a)}<smiles>Cn1cc(I)c2ccccc21</smiles>

Following general procedure D, starting from commercially available 1methylindole $(0.43 \mathrm{~mL}, 3.4 \mathrm{mmol})$ and 16 , a purification by column chromatography (DCM/MeOH 19:1) afford the title compound 5a (0.79 g, $2.1 \mathrm{mmol}, 61 \%$ yield) (CAS number 2130906-04-4) as an off-white solid.

${ }^{1} \mathbf{H}$ NMR $\left(400 \mathrm{MHz}, \mathrm{CDCl}_{3}\right) \delta 8.43\left(\mathrm{~d}, J=7.5 \mathrm{~Hz}, 1 \mathrm{H}, \underline{\mathrm{H}}_{\mathrm{Ar}}\right), 7.77(\mathrm{~s}, 1 \mathrm{H}, \mathrm{C} \underline{\mathrm{H}}-\mathrm{N})$, 7.56-7.49 (m, 2H, $\left.\underline{\mathrm{H}}_{\mathrm{Ar}}\right), 7.47-7.41\left(\mathrm{~m}, 2 \mathrm{H}, \underline{\mathrm{H}}_{\mathrm{Ar}}\right), 7.35-7.26\left(\mathrm{~m}, 2 \mathrm{H}, \underline{\mathrm{H}}_{\mathrm{Ar}}\right), 6.85\left(\mathrm{~d}, J=8.2 \mathrm{~Hz}, 1 \mathrm{H}, \underline{\mathrm{H}}_{\mathrm{Ar}}\right), 4.02$ (s, $\left.3 \mathrm{H}, \mathrm{NCH}_{3}\right)$.

${ }^{13}$ C NMR $\left(101 \mathrm{MHz}, \mathrm{CDCl}_{3}\right) \delta 167.0,138.7,137.7,133.6,133.4,132.7,130.7,129.5,125.4,124.5,122.8$, $120.1,116.3,110.9,79.2,34.1$. Spectra data matched with the values reported in literature. ${ }^{12}$

${ }^{14}$ Parsons, A. T.; Buchwald, S. L. Angew. Chem. Int. Ed. 2011, 50, 9120-9123

15 Caramenti, P.; Nicolai, S.; Waser, J. Chem. Eur. J. 2017, 23,14702-14706 
<smiles>CCCCCn1cc(I)c2ccccc21</smiles>

Following general procedure $\mathbf{D}$, starting from $\mathbf{1 5 b}(257 \mathrm{mg}, 1.37 \mathrm{mmol})$ and $\mathbf{1 6}$, a purification by column chromatography (DCM/MeOH 19:1) afford the title compound $\mathbf{5 b}$ (357 $\mathrm{mg}, 0.820 \mathrm{mmol}, 60 \%$ yield) as an off-white solid. $\mathbf{~ m p ~ 1 9 4 - ~}$ $196^{\circ} \mathrm{C}$. Rf 0.37 (DCM/MeOH 19:1).

${ }^{1} \mathbf{H}$ NMR $(400 \mathrm{MHz}, \mathrm{MeOD}) \delta 8.26\left(\mathrm{~d}, J=6.6 \mathrm{~Hz}, 1 \mathrm{H}, \underline{\mathrm{H}}_{\mathrm{Ar}}\right), 8.15(\mathrm{~s}, 1 \mathrm{H}, \mathrm{C} \underline{\mathrm{H}}-$ $\mathrm{N}), 7.70\left(\mathrm{~d}, J=8.3 \mathrm{~Hz}, 1 \mathrm{H}, \underline{\mathrm{H}}_{\mathrm{Ar}}\right), 7.60\left(\mathrm{t}, J=7.3 \mathrm{~Hz}, 1 \mathrm{H}, \underline{\mathrm{H}}_{\mathrm{Ar}}\right), 7.48\left(\mathrm{~d}, J=8.0 \mathrm{~Hz}, 1 \mathrm{H}, \underline{\mathrm{H}}_{\mathrm{Ar}}\right), 7.44-7.37(\mathrm{~m}$, $\left.2 \mathrm{H}, \underline{\mathrm{H}}_{\mathrm{Ar}}\right), 7.29\left(\mathrm{t}, J=7.5 \mathrm{~Hz}, 1 \mathrm{H}, \underline{\mathrm{H}}_{\mathrm{Ar}}\right), 6.87\left(\mathrm{~d}, J=8.3 \mathrm{~Hz}, 1 \mathrm{H}, \underline{\mathrm{H}}_{\mathrm{Ar}}\right), 4.42\left(\mathrm{t}, J=7.0 \mathrm{~Hz}, 2 \mathrm{H}, \mathrm{NC}_{2}\right), 2.03-$ 1.90 (m, 2H, $\left.\underline{\mathrm{H}}_{\text {aliph }}\right), 1.47-1.24$ (m, 4H, $\left.\underline{\mathrm{H}}_{\text {aliph }}\right), 0.91\left(\mathrm{t}, J=6.9 \mathrm{~Hz}, 3 \mathrm{H}, \mathrm{C}_{3}\right)$.

${ }^{13}$ C NMR (101 MHz, MeOD) $\delta 170.3,140.2,138.5,135.0,134.5,133.2,131.7,130.6,127.7,125.1,123.5$, $120.6,116.8,112.4,77.9,48.3,30.8,30.1,23.3,14.3$.

IR $v_{\max } 2927$ (w), 1596 (s), 1555 (s), 1435 (m), 1340 (s), 1292 (m), 826 (s), 744 (s), 583 (s).

HRMS calculated for $\mathrm{C}_{20} \mathrm{H}_{21} \mathrm{INO}_{2}{ }^{+}[\mathrm{M}+\mathrm{H}]^{+}$434.0612; found 434.0622.

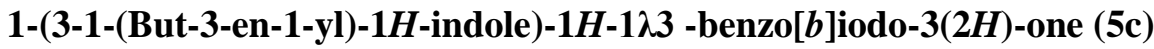<smiles>O=C1OI(I)C2=CN3CCC=C2c2cccc1c23</smiles>

Following general procedure D, starting from 15c (390 mg, $2.28 \mathrm{mmol})$ and 16, a purification by column chromatography (DCM/MeOH 32:1) afford the title compound $\mathbf{5 c}$ (501 $\mathrm{mg}, 1.16 \mathrm{mmol}, 53 \%$ yield) as a pale yellow solid.

${ }^{1} \mathbf{H}$ NMR $\left(400 \mathrm{MHz}, \mathrm{CDCl}_{3}\right) \delta 8.45\left(\mathrm{~d}, J=7.3 \mathrm{~Hz}, 1 \mathrm{H}, \underline{\mathrm{H}}_{\mathrm{Ar}}\right), 7.73-7.68(\mathrm{~m}, 1 \mathrm{H}$, $\left.\underline{\mathrm{H}}_{\mathrm{Ar}}\right), 7.57-7.51\left(\mathrm{~m}, 2 \mathrm{H}, \mathrm{C} \underline{\mathrm{H}}-\mathrm{N}+\underline{\mathrm{H}}_{\mathrm{Ar}}\right), 7.48-7.42\left(\mathrm{~m}, 2 \mathrm{H}, \underline{\mathrm{H}}_{\mathrm{Ar}}\right), 7.33-7.28(\mathrm{~m}$,

$\left.2 \mathrm{H}, \underline{\mathrm{H}}_{\mathrm{Ar}}\right), 6.77\left(\mathrm{~d}, J=8.3 \mathrm{~Hz}, 1 \mathrm{H}, \underline{\mathrm{H}}_{\mathrm{Ar}}\right), 5.80\left(\mathrm{ddt}, J=17.1,10.2,6.9 \mathrm{~Hz}, 1 \mathrm{H}, \mathrm{C} \underline{\mathrm{H}}=\mathrm{CH}_{2}\right), 5.11-5.04(\mathrm{~m}, 2 \mathrm{H}$, $\left.\mathrm{CH}=\mathrm{C}_{2}\right), 4.39\left(\mathrm{t}, J=6.8 \mathrm{~Hz}, 2 \mathrm{H}, \mathrm{NC}_{2}\right), 2.71\left(\mathrm{q}, J=6.8 \mathrm{~Hz}, 2 \mathrm{H}, \mathrm{NCH}_{2} \mathrm{C}_{2}\right)$.

${ }^{13}$ C NMR $\left(101 \mathrm{MHz}, \mathrm{CDCl}_{3}\right) \delta 166.9,137.6,137.5,136.9,133.6,133.6,133.4,132.8,130.8,129.6,125.3$, $124.5,122.8,120.3,118.9,116.4,111.0,79.7,47.1,34.3$. Spectra data matched with the values reported in literature. ${ }^{12}$

\section{1-(3-5-Bromo-1-methyl-1H-indole)-1H-1 $\lambda_{3}$-benzo[b]iodo-3(2H)-one (5d)}<smiles>Cn1cc(I)c2cc(Br)ccc21</smiles>

Following general procedure D, starting from $\mathbf{1 5 d}(406 \mathrm{mg}, 1.93 \mathrm{mmol})$ and $\mathbf{1 6}$, a purification by column chromatography (DCM/MeOH 19:1) afford the title compound 5d (470 mg, $1.03 \mathrm{mmol}, 53 \%$ yield) as an off-white solid. mp 223-225 ${ }^{\circ} \mathrm{C}$. Rf 0.21 (DCM/MeOH 19:1).

${ }^{1} \mathbf{H}$ NMR $\left(400 \mathrm{MHz}, \mathrm{CD}_{2} \mathrm{Cl}_{2}\right) \delta 8.33\left(\mathrm{~d}, J=7.4 \mathrm{~Hz}, 1 \mathrm{H}, \underline{\mathrm{H}}_{\mathrm{Ar}}\right), 7.70(\mathrm{~s}, 1 \mathrm{H}, \mathrm{C} \underline{\mathrm{H}}-\mathrm{N})$, $7.63\left(\mathrm{~s}, 1 \mathrm{H}, \underline{\mathrm{H}}_{\mathrm{Ar}}\right), 7.57\left(\mathrm{t}, J=7.3 \mathrm{~Hz}, 1 \mathrm{H}, \underline{\mathrm{H}}_{\mathrm{Ar}}\right), 7.52\left(\mathrm{dd}, J=8.8,1.6 \mathrm{~Hz}, 1 \mathrm{H}, \underline{\mathrm{H}}_{\mathrm{Ar}}\right)$, $7.44\left(\mathrm{~d}, J=8.8 \mathrm{~Hz}, 1 \mathrm{H}, \underline{\mathrm{H}}_{\mathrm{Ar}}\right), 7.34\left(\mathrm{t}, J=7.1 \mathrm{~Hz}, 1 \mathrm{H}, \underline{\mathrm{H}}_{\mathrm{Ar}}\right), 6.84\left(\mathrm{~d}, J=8.3 \mathrm{~Hz}, 1 \mathrm{H}, \underline{\mathrm{H}}_{\mathrm{Ar}}\right), 3.96\left(\mathrm{~s}, 3 \mathrm{H}, \mathrm{NC}_{3}\right)$. ${ }^{13}$ C NMR $\left(101 \mathrm{MHz}, \mathrm{CD}_{2} \mathrm{Cl}_{2}\right) \delta 166.6,139.9,136.9,133.9,133.7,132.5,131.5,131.0,127.5,125.7,123.0$, $116.7,116.2,112.8,79.5,34.4$.

IR $v_{\max } 3075(\mathrm{~m}), 1601(\mathrm{~s}), 1495(\mathrm{~s}), 1347$ (s), 740 (s), 685 (m).

HRMS calculated for $\mathrm{C}_{16} \mathrm{H}_{12}{ }^{79} \mathrm{BrINO}_{2}{ }^{+}[\mathrm{M}+\mathrm{H}]^{+} 455.9091$; found 455.9093 . 
<smiles>O=C1OI(c2c[nH]c3ccccc23)c2ccccc21</smiles>

Following general procedure $\mathbf{D}$ and remplacing $\mathrm{Zn}(\mathrm{OTf})_{2}$ by $\mathrm{Sc}(\mathrm{OTf})_{3}$, starting from 15e $(579 \mathrm{mg}, 2.50 \mathrm{mmol})$ and $\mathbf{1 6}$, a purification by column chromatography (DCM/MeOH 5:1) afforded the title compound 5 e (527 mg, $1.45 \mathrm{mmol}, 58 \%$ yield) as a pale beige solid.

${ }^{1}$ H NMR (400 MHz, DMSO-d6) $\delta 12.36$ (s, 1H, NH), 8.26 (s, 1H, CH-N), 8.12 (d, $\left.J=7.3 \mathrm{~Hz}, 1 \mathrm{H}, \underline{\mathrm{H}}_{\mathrm{Ar}}\right), 7.64\left(\mathrm{~d}, J=8.2 \mathrm{~Hz}, 1 \mathrm{H}, \underline{\mathrm{H}}_{\mathrm{Ar}}\right), 7.56\left(\mathrm{t}, J=7.3 \mathrm{~Hz}, 1 \mathrm{H}, \underline{\mathrm{H}}_{\mathrm{Ar}}\right), 7.49(\mathrm{~d}, J=7.9 \mathrm{~Hz}, 1 \mathrm{H}$, $\left.\underline{\mathrm{H}}_{\mathrm{Ar}}\right), 7.41\left(\mathrm{t}, J=7.6 \mathrm{~Hz}, 1 \mathrm{H}, \underline{\mathrm{H}}_{\mathrm{Ar}}\right), 7.31\left(\mathrm{t}, J=7.6 \mathrm{~Hz}, 1 \mathrm{H}, \underline{\mathrm{H}}_{\mathrm{Ar}}\right), 7.20\left(\mathrm{t}, J=7.5 \mathrm{~Hz}, 1 \mathrm{H}, \underline{\mathrm{H}}_{\mathrm{Ar}}\right), 6.76(\mathrm{~d}, J=$ $\left.8.2 \mathrm{~Hz}, 1 \mathrm{H}, \underline{\mathrm{H}}_{\mathrm{Ar}}\right)$.

${ }^{13}$ C NMR (101 MHz, DMSO-d6) $\delta$ 165.8, 136.5 (2C), 134.6, 133.1, 131.3, 130.1, 128.6, 126.2, 123.3,

121.6, 119.2, 116.0, 112.9, 80.3. Spectra data matched with the values reported in literature. ${ }^{12}$

\section{1-(3-1-Methyl-1H-pyrrole)-1H-1 $1 \lambda_{3}$-benzo[b]iodo-3(2H)-one (5f)}

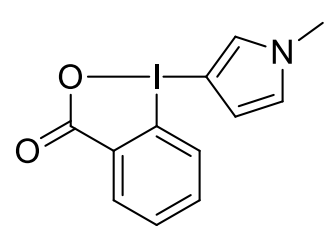

Following general procedure $\mathbf{D}$, starting from 1-methylpyrrole $(0.37 \mathrm{~mL}, 4.2 \mathrm{mmol})$ and 16, a purification by column chromatography (EtOAc/MeOH 9:1) afford the title compound $\mathbf{5 f}$ (763 mg, $2.33 \mathrm{mmol}, 56 \%$ yield) (CAS number 2130906-17-9) as a off-white solid and 1-(2-1-methyl- $1 H$-pyrrole)-1 $H$-1 $\lambda_{3}$-benzo[ $\left.b\right]$ iodo-3( $\left.2 H\right)$-one (525 mg, $1.64 \mathrm{mmol}, 39 \%$ yield) (CAS number 2130906-18-0) as a pale brown solid. ${ }^{1}$ H NMR (400 MHz, MeOD) $\delta 8.25\left(\mathrm{dd}, J=7.5,1.6 \mathrm{~Hz}, 1 \mathrm{H}, \underline{\mathrm{H}}_{\mathrm{Ar}}\right), 7.64(\mathrm{t}, J=7.3$ $\left.\mathrm{Hz}, 1 \mathrm{H}, \underline{\mathrm{H}}_{\mathrm{Ar}}\right), 7.55\left(\mathrm{td}, J=7.8,7.3,1.7 \mathrm{~Hz}, 1 \mathrm{H}, \underline{\mathrm{H}}_{\mathrm{Ar}}\right), 7.48-7.45\left(\mathrm{~m}, 1 \mathrm{H}, \underline{\mathrm{H}}_{\mathrm{Ar}}\right), 7.12\left(\mathrm{~d}, J=8.2 \mathrm{~Hz}, 1 \mathrm{H}, \underline{\mathrm{H}}_{\mathrm{Ar}}\right)$, 7.06-7.02 (m, $\left.1 \mathrm{H}, \underline{\mathrm{H}}_{\mathrm{Ar}}\right), 6.62\left(\mathrm{dd}, J=2.8,1.7 \mathrm{~Hz}, 1 \mathrm{H}, \underline{\mathrm{H}}_{\mathrm{Ar}}\right), 3.87\left(\mathrm{~s}, 3 \mathrm{H}, \mathrm{NC}_{3}\right)$.

${ }^{13}$ C NMR (101 MHz, MeOD) $\delta 170.3,135.3,133.8,133.5,133.0,131.7,128.1,127.8,117.4,116.7,82.4$, 37.1. Spectra data matched with the values reported in literature. ${ }^{11}$ 


\section{Optimization of the transition metal-catalyzed $\mathrm{C}-\mathrm{H}$ activation}

4a (9.8 mg, $80 \mu \mathrm{mol}, 1.0$ equiv.), 5a (30.3 mg, $80.0 \mu \mathrm{mol}, 1.00$ equiv.), metal catalyst (2.0 mg, 2.5 $\mu \mathrm{mol}, 3 \mathrm{~mol} \%$ ) and base (see Table 1 below) were solubilized in dry $\mathrm{MeOH}$ [0.1 M]. The mixture was stirred at room temperature for 10 minutes. The suspension was diluted with DCM (5 mL) and quenched with a saturated aqueous solution of $\mathrm{NaHCO}_{3}(5 \mathrm{~mL})$. The two layers were separated and the aqueous layer was extracted twice with DCM $(5 \mathrm{~mL})$. The organic layers were combined, dried over magnesium sulfate dehydrate, filtered and concentrated under reduced pressure. The crude residue was purified by column chromatography on silica gel using Pentane/EtOAc 85:15 as eluent to afford the pure title compound.

Table 1: Optimization for the iridium-catalyzed 3-acylation with salicylaldehyde 4a and Me-indoleBX 5a<smiles>O=Cc1ccccc1O</smiles>

$4 a$<smiles>Cn1cc(I)c2ccccc21</smiles>

$5 a$
$\left[\mathrm{MCp}^{*} \mathrm{Cl}_{2}\right]_{2}(3 \mathrm{~mol} \%)$,
base $(0.1-1.2$ equiv)

$\mathrm{MeOH}[0.1 \mathrm{M}], \mathrm{T}$<smiles>Cn1cc(C(=O)c2ccccc2O)c2ccccc21</smiles>

$6 a$

\begin{tabular}{|c|c|c|c|}
\hline Catalyst & Temperature $\left({ }^{\circ} \mathbf{C}\right)$ & Base & Isolated yield (\%) \\
\hline- & 80 & CsOAc (1.2 equiv) & NR \\
\hline$\left[\mathrm{RhCp}^{*} \mathrm{Cl}_{2}\right]_{2}$ & 80 & CsOAc ( 1.2 equiv) & $<20$ \\
\hline$\left[\mathrm{IrCp} * \mathrm{Cl}_{2}\right]_{2}$ & $\mathrm{rt}$ & CsOAc (1.2 equiv) & 91 \\
\hline$\left[\mathrm{IrCp} * \mathrm{Cl}_{2}\right]_{2}$ & $\mathrm{rt}$ & $\mathrm{NaOAc}$ ( 1.2 equiv) & 90 \\
\hline$\left[\mathrm{IrCp} * \mathrm{Cl}_{2}\right]_{2}$ & $\mathrm{rt}$ & KOAc (1.2 equiv) & 90 \\
\hline$\left[\mathrm{IrCp} * \mathrm{Cl}_{2}\right]_{2}$ & $\mathrm{rt}$ & KOAc (1.0 equiv) & 90 \\
\hline$\left[\mathrm{IrCp} * \mathrm{Cl}_{2}\right]_{2}$ & $\mathrm{rt}$ & KOAc ( 0.5 equiv) & $86^{\mathrm{a}}$ \\
\hline$\left[\mathrm{IrCp} * \mathrm{Cl}_{2}\right]_{2}$ & $\mathrm{rt}$ & - & $56^{\mathrm{a}}$ \\
\hline$\left[\mathrm{IrCp}^{*} \mathrm{Cl}_{2}\right]_{2}(1 \mathrm{~mol} \%)$ & $\mathrm{rt}$ & KOAc (1.0 equiv) & 94 \\
\hline$\left[\mathrm{IrCp}^{*} \mathrm{Cl}_{2}\right]_{2}(1 \mathrm{~mol} \%)$ & 80 & KOAc (1.0 equiv) & $53^{\mathrm{b}}$ \\
\hline$\left[\mathrm{IrCp}^{*} \mathrm{Cl}_{2}\right]_{2}(1 \mathrm{~mol} \%)$ & 80 & KOAc (1.0 equiv) & $59^{c}$ \\
\hline$\left[\mathrm{IrCp} * \mathrm{Cl}_{2}\right]_{2}$ & 80 & KOAc (1.0 equiv) & decomposition $^{\mathrm{d}, \mathrm{e}}$ \\
\hline$\left[\mathrm{IrCp} * \mathrm{Cl}_{2}\right]_{2}$ & 50 & KOAc (1.0 equiv) & decomposition $^{\mathrm{f}, \mathrm{e}}$ \\
\hline$\left[\mathrm{IrCp}^{*} \mathrm{Cl}_{2}\right]_{2}$ & 50 & KOAc (1.0 equiv) & decomposition ${ }^{\mathrm{g}, \mathrm{e}}$ \\
\hline
\end{tabular}

Table 2: Optimization for the rhodium-catalyzed 3-acylation with $\mathrm{N}$-(2-formylphenyl)-4methylbenzenesulfonamide 7a and Me-indoleBX 5a

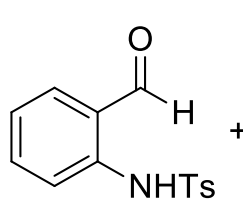

$7 \mathrm{a}$<smiles>Cn1cc(I)c2ccccc21</smiles>

$5 a$
$\left[\mathrm{MCp}{ }^{*} \mathrm{Cl}_{2}\right]_{2}(3 \mathrm{~mol} \%)$, base (0.1-1.2 equiv)<smiles>Cn1c(C(=O)c2ccccc2N)cnc1-c1ccccn1</smiles>

$8 \mathrm{a}$

\begin{tabular}{lccc}
\hline \multicolumn{1}{c}{ Catalyst } & Temperature $\left({ }^{\circ} \mathbf{C}\right)$ & Solvent & Isolated yield (\%) \\
\hline- & 80 & DCE & NR \\
{$\left[\mathrm{IrCp} * \mathrm{Cl}_{2}\right]_{2}$} & $\mathrm{rt}$ & $\mathrm{DCE}$ & 75 \\
{$\left[\mathrm{RhCp} * \mathrm{Cl}_{2}\right]_{2}$} & $\mathrm{rt}$ & $\mathrm{DCE}$ & 78 \\
{$\left[\mathrm{RhCp}^{*} \mathrm{Cl}_{2}\right]_{2}$} & $\mathrm{rt}$ & $\mathrm{MeOH}$ & 82 \\
{$\left[\mathrm{RhCp}^{*} \mathrm{Cl}_{2}\right]_{2}$} & 40 & $\mathrm{MeOH}$ & 86 \\
{$\left[\mathrm{RhCp} * \mathrm{Cl}_{2}\right]_{2}(1 \mathrm{~mol} \%)$} & 40 & $\mathrm{MeOH}$ & 89 \\
\hline
\end{tabular}




\section{Mechanism proposal}

Scheme S1: Plausible mechanism of the iridium-catalyzed C-H functionalization for salicylaldehyde 4a and Me-indoleBX 5a

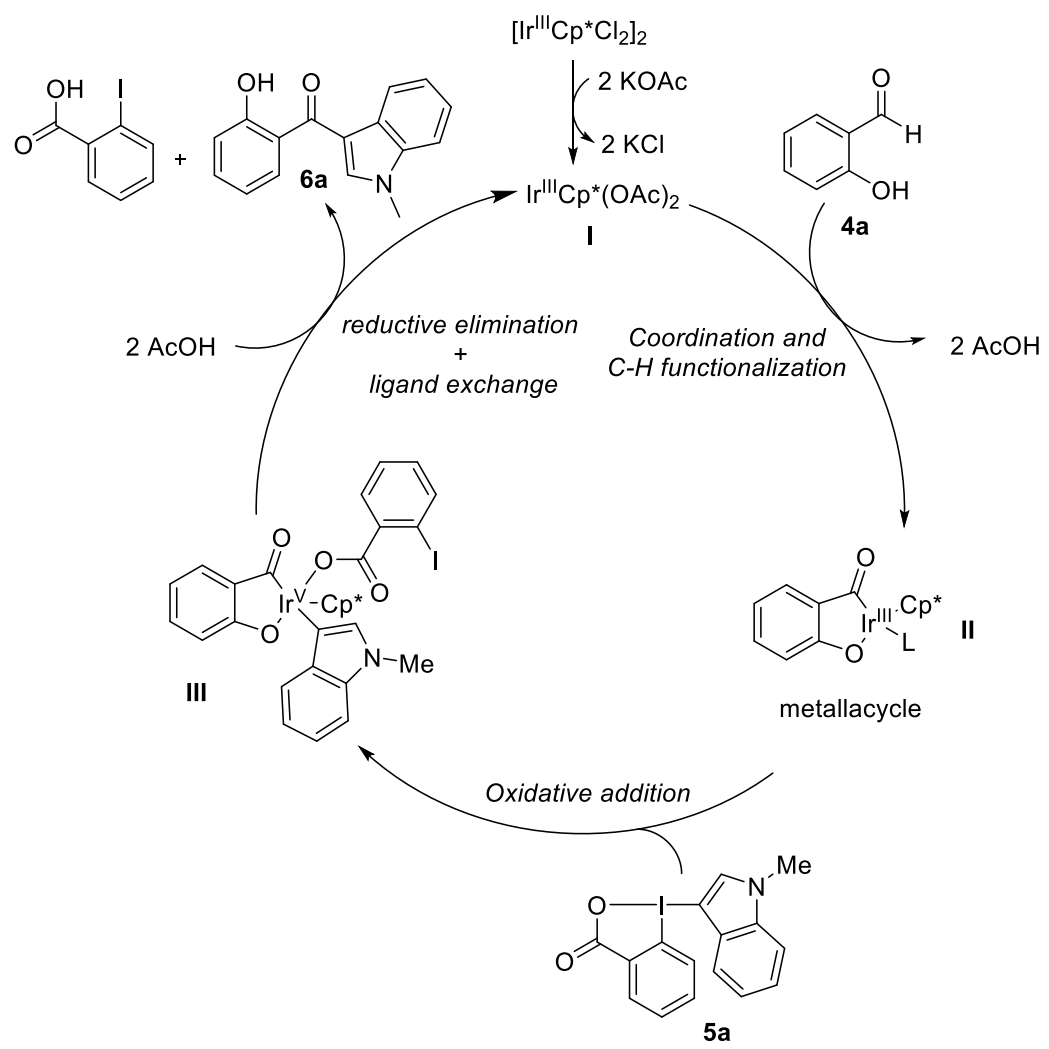


Scheme S2: Plausible mechanism of the rhodium-catalyzed C-H functionalization for $N$-(2formylphenyl)-4-methylbenzenesulfonamide 7a and Me-indoleBX 5a

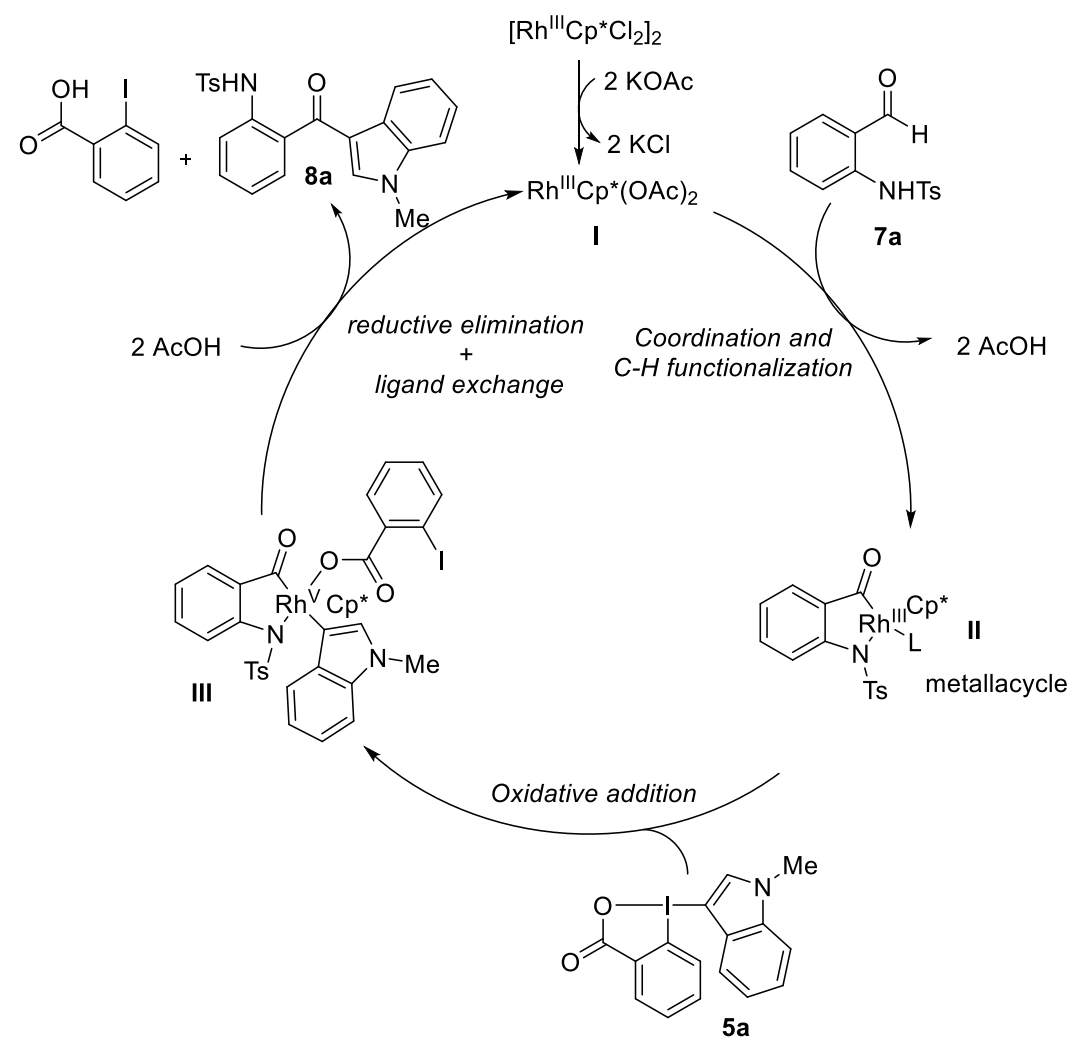

Discussion: A very similar catalytic cycle can be proposed for both the iridium and rhodium catalyst. Ligand exchange with potassium acetate first gives the active catalyst, which reacts than with either $\mathbf{4 a}$ or 7a via coordination and $\mathrm{C}$-H activation to form metallacycle II and two equivalents of acetic acid. In fact, metallacycle II bearing a rhodium center and $\mathrm{L}=$ pyridine or $\mathrm{MeCN}$ could be isolated in excellent yields using similar reaction conditions. ${ }^{16}$ In the second step, oxidative addition of hypervalent iodine reagent 5a onto metallacycle II gives high valent metal intermediate III. Finally, reductive elimination and ligand exchange regenerate the metal acetate complex. This mechanism is in accordance with what was proposed for alkynylation. ${ }^{16}$ Further work will be done to fully elucidate the mechanism of this transformation.

${ }^{16}$ Wang, H.; Xie, F.; Qi, Z.; Li, X. Org. Lett. 2015, 17, 920. 


\section{Scope}

\subsection{General procedure $\mathrm{E} 1$ for the synthesis of 3-salicyloylindoles}<smiles>[R]c1ccc2c(C(=O)c3cn([R2])c4ccc([R])cc34)c(O)ccc2c1</smiles>

[ $\left.\mathrm{IrCp}^{*} \mathrm{Cl}_{2}\right]_{2}(1.2 \mathrm{mg}, 1.5 \mu \mathrm{mol}, 1 \mathrm{~mol} \%)$, potassium acetate $(14.8 \mathrm{mg}, 0.150 \mathrm{mmol}, 1.00$ equiv), corresponding salicylaldehyde $(0.15 \mathrm{mmol}, 1.00$ equiv $)$ and the corresponding hypervalent iodine reagent $(0.15 \mathrm{mmol}, 1.00$ equiv) were solubilized in dry $\mathrm{MeOH}(1.5 \mathrm{~mL}, 0.1 \mathrm{M})$. The mixture was stirred at room temperature for 10 minutes. The suspension was diluted with DCM $(5 \mathrm{~mL})$ and quenched with a saturated aqueous solution of $\mathrm{NaHCO}_{3}(5 \mathrm{~mL})$. The two layers were separated and the aqueous layer was extracted twice with DCM $(5 \mathrm{~mL})$. The organic layers were combined, dried over magnesium sulfate dehydrate, filtered and concentrated under reduced pressure. The crude residue was purified by column chromatography on silica gel using the indicated solvents to afford the pure title compound.

\subsection{General procedure E2 for the synthesis of 3-(2-sulfonamino)benzoylindoles}<smiles>[R][R]OS(=O)Nc1cccc([R])c1C(=O)c1cn([R2])c2ccc([R3])cc12</smiles>

[RhCp* $\left.\mathrm{Cl}_{2}\right]_{2}(0.93 \mathrm{mg}, 1.5 \mu \mathrm{mol}, 1 \mathrm{~mol} \%)$, potassium acetate (14.8 mg, $\left.0.150 \mathrm{mmol}, 1.00 \mathrm{equiv}\right)$, corresponding salicylaldehyde $(0.15 \mathrm{mmol}, 1.00$ equiv) and corresponding hypervalent iodine reagent ( $0.15 \mathrm{mmol}, 1.00$ equiv) were solubilized in dry $\mathrm{MeOH}(1.5 \mathrm{~mL}, 0.1 \mathrm{M})$. The mixture was stirred at 40 ${ }^{\circ} \mathrm{C}$ for 4 hours. The suspension was diluted with DCM $(5 \mathrm{~mL})$ and quenched with a saturated aqueous solution of $\mathrm{NaHCO}_{3}(5 \mathrm{~mL})$. The two layers were separated and the aqueous layer was extracted twice with DCM ( $5 \mathrm{~mL})$. The organic layers were combined, dried over magnesium sulfate dehydrate, filtered and concentrated under reduced pressure. The crude residue was purified by column chromatography on silica gel using the indicated solvents to afford the pure title compound. 
<smiles>Cn1cc(C(=O)c2ccccc2O)c2ccccc21</smiles>

Following General Procedure E1, starting from commercially available salicylaldehyde and Me-indoleBX 5a, a purification by column chromatography on silica gel (Pentane/EtOAc 85:15) afford the title compound 6a (35.4 mg, 0.140 mmol, 94\% yield) or (281 mg, $1.12 \mathrm{mmol}, 93 \%$ yield) as a yellow solid. mp 128$130{ }^{\circ} \mathrm{C}$. Rf 0.46 (Pentane/EtOAc 4:1).

$1_{\mathbf{H}}$ NMR $\left(400 \mathrm{MHz}, \mathrm{CDCl}_{3}\right) \delta 12.06$ (bs, $\left.1 \mathrm{H}, \mathrm{OH}\right), 8.33-8.28\left(\mathrm{~m}, 1 \mathrm{H}, \underline{\mathrm{H}}_{\mathrm{Ar}}\right), 7.84(\mathrm{dd}, J=7.9,1.6 \mathrm{~Hz}, 1 \mathrm{H}$, $\left.\underline{\mathrm{H}}_{\mathrm{Ar}}\right), 7.62(\mathrm{~s}, 1 \mathrm{H}, \mathrm{C} \underline{\mathrm{H}}-\mathrm{N}), 7.46\left(\mathrm{ddd}, J=8.6,7.4,1.6 \mathrm{~Hz}, 1 \mathrm{H}, \underline{\mathrm{H}}_{\mathrm{Ar}}\right), 7.40-7.32\left(\mathrm{~m}, 3 \mathrm{H}, \underline{\mathrm{H}}_{\mathrm{Ar}}\right), 7.06(\mathrm{~d}, J=8.3$ $\left.\mathrm{Hz}, 1 \mathrm{H}, \underline{\mathrm{H}}_{\mathrm{Ar}}\right), 6.92\left(\mathrm{t}, J=8.0 \mathrm{~Hz}, 1 \mathrm{H}, \underline{\mathrm{H}}_{\mathrm{Ar}}\right), 3.86\left(\mathrm{~s}, 3 \mathrm{H}, \mathrm{NC}_{3}\right)$.

13C NMR (101 MHz, $\left.\mathrm{CDCl}_{3}\right) \delta$ 193.5, 162.0, 137.4, 137.0, 134.7, 131.5, 127.3, 123.9, 122.8, 122.4, 121.4, $118.7,118.2,114.7,109.9,33.8$.

IR $v_{\max } 1586$ (s), 1521 (s), 1463 (s), 1370 (s), 1330 (m), 1276 (m), 1236 (s), 1200 (s), 1154 (s), 1128 (s), 1099 (s), 887 (s), 743 (s), 663 (s).

HRMS calculated for $\mathrm{C}_{16} \mathrm{H}_{13} \mathrm{NO}_{2}{ }^{+}[\mathrm{M}+\mathrm{H}]^{+}$252.1019; found 252.1022 .

(2-Hydroxy-5-methylphenyl)(1-methyl-1H-indol-3-yl)methanone (6b)<smiles>Cc1ccc(O)c(C(=O)c2cn(C)c3ccccc23)c1</smiles>

Following General Procedure E1, starting from commercially available 2-hydroxy5-methylbenzaldehyde and Me-indoleBX 5a, a purification by column chromatography on silica gel (Pentane/EtOAc 85:15) afford the title compound $\mathbf{6 b}$ (35.4 mg, $0.134 \mathrm{mmol}, 89 \%$ yield) as a yellow solid. $\mathbf{m p ~} 170-172{ }^{\circ} \mathrm{C}$. $\mathbf{R f} 0.40$ (Pentane/EtOAc 4:1).

${ }^{1} \mathbf{H}$ NMR $\left(400 \mathrm{MHz}, \mathrm{CDCl}_{3}\right) \delta 11.82(\mathrm{bs}, 1 \mathrm{H}, \mathrm{OH}), 8.30-8.26\left(\mathrm{~m}, 1 \mathrm{H}, \underline{\mathrm{H}}_{\mathrm{Ar}}\right), 7.63\left(\mathrm{~d}, J=1.4 \mathrm{~Hz}, 1 \mathrm{H}, \underline{\mathrm{H}}_{\mathrm{Ar}}\right)$, $7.61(\mathrm{~s}, 1 \mathrm{H}, \mathrm{C} \underline{\mathrm{H}}-\mathrm{N}), 7.39-7.31\left(\mathrm{~m}, 3 \mathrm{H}, \underline{\mathrm{H}}_{\mathrm{Ar}}\right), 7.27\left(\mathrm{dd}, J=8.7,2.0 \mathrm{~Hz}, 1 \mathrm{H}, \underline{\mathrm{H}}_{\mathrm{Ar}}\right), 6.96(\mathrm{~d}, J=8.4 \mathrm{~Hz}, 1 \mathrm{H}$, $\left.\underline{\mathrm{H}}_{\mathrm{Ar}}\right), 3.87\left(\mathrm{~s}, 3 \overline{\mathrm{H}}, \mathrm{NC}_{3}\right), 2.33\left(\mathrm{~s}, 3 \mathrm{H}, \underline{\mathrm{CH}}_{3}\right)$.

${ }^{13} \mathrm{C}$ NMR $\left(101 \mathrm{MHz}, \mathrm{CDCl}_{3}\right) \delta 193.6,159.8,137.4,136.8,135.6,131.4,127.7,127.4,123.8,122.7,122.5$, $121.2,117.9,114.8,109.9,33.7,20.7$.

IR $v_{\max } 2913$ (w), 1564 (s), 1521 (s), 1469 (s), 1343 (s), 1219 (s), 1109 (s), 823 (s), 748 (s).

HRMS calculated for $\mathrm{C}_{17} \mathrm{H}_{16} \mathrm{NO}_{2}{ }^{+}[\mathrm{M}+\mathrm{H}]^{+} 266.1176$; found 266.1177 .

\section{(2-Hydroxy-5-methoxyphenyl)(1-methyl-1H-indol-3-yl)methanone (6c)}

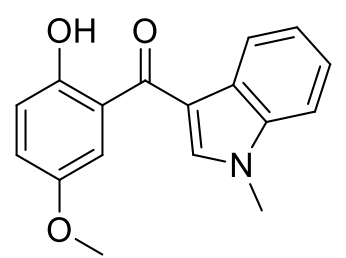

Following General Procedure E1, starting from commercially available 2-hydroxy5-methoxybenzaldehyde and Me-indoleBX 5a, a purification by column chromatography on silica gel (Pentane/EtOAc 7:3) afford the title compound 6c (38.0 mg, $0.135 \mathrm{mmol}, 90 \%$ yield) as a yellow solid. mp $147-149{ }^{\circ} \mathrm{C}$. Rf 0.24 (Pentane/EtOAc 4:1).

1H NMR $\left(400 \mathrm{MHz}, \mathrm{CDCl}_{3}\right) \delta 11.45(\mathrm{bs}, 1 \mathrm{H}, \mathrm{OH}), 8.31-8.27\left(\mathrm{~m}, 1 \mathrm{H}, \underline{\mathrm{H}}_{\mathrm{Ar}}\right), 7.68(\mathrm{~s}$, $1 \mathrm{H}, \mathrm{C} \underline{\mathrm{H}}-\mathrm{N}), 7.41-7.33\left(\mathrm{~m}, 4 \mathrm{H}, \underline{\mathrm{H}}_{\mathrm{Ar}}\right), 7.10\left(\mathrm{dd}, J=9.0,3.1 \mathrm{~Hz}, 1 \mathrm{H}, \underline{\mathrm{H}}_{\mathrm{Ar}}\right), 7.01\left(\mathrm{~d}, J=9.0 \mathrm{~Hz}, 1 \mathrm{H}, \underline{\mathrm{H}}_{\mathrm{Ar}}\right), 3.88$ (s, $\left.3 \mathrm{H}, \mathrm{OCH}_{3}\right), 3.79$ (s, $\left.3 \mathrm{H}, \mathrm{NCH}_{3}\right)$.

${ }^{13} \mathrm{C}$ NMR $\left(101 \mathrm{MHz}, \mathrm{CDCl}_{3}\right) \delta 193.1,155.9,151.7,137.5,136.8,127.3,123.9,122.9,122.5,121.4,121.2$, $118.8,115.9,114.9,110.0,56.2,33.8$.

IR $v_{\max } 3102(\mathrm{w}), 2927(\mathrm{~m}), 2852(\mathrm{w}), 1562$ (s), 1520 (s), 1484 (s), 1460 (s), 1221 (s), 1101 (s), 1035 (s), $828(\mathrm{~s}), 742(\mathrm{~s}), 670(\mathrm{~s})$.

HRMS calculated for $\mathrm{C}_{17} \mathrm{H}_{16} \mathrm{NO}_{3}{ }^{+}[\mathrm{M}+\mathrm{H}]^{+}$282.1125; found 282.1128 . 
<smiles>Cn1cc(C(=O)c2cc(F)ccc2O)c2ccccc21</smiles>

Following General Procedure E1, starting from commercially available 5-fluoro-2hydroxybenzaldehyde and Me-indoleBX 5a, a purification by column chromatography on silica gel (Pentane/EtOAc 85:15) afford the title compound $\mathbf{6 d}$ (36.3 mg, $0.135 \mathrm{mmol}, 90 \%$ yield) as a yellow solid. mp $160-162{ }^{\circ} \mathrm{C}$. Rf 0.51 (Pentane/EtOAc 4:1).

${ }^{1} \mathbf{H}$ NMR $\left(400 \mathrm{MHz}, \mathrm{CDCl}_{3}\right) \delta 11.71(\mathrm{~s}, 1 \mathrm{H}, \mathrm{OH}), 8.32-8.29\left(\mathrm{~m}, 1 \mathrm{H}, \underline{\mathrm{H}}_{\mathrm{Ar}}\right), 7.65(\mathrm{~s}, 1 \mathrm{H}, \mathrm{C} \underline{\mathrm{H}}-\mathrm{N}), 7.54$ (dd, $J$ $\left.=9.0,3.1 \mathrm{~Hz}, 1 \mathrm{H}, \underline{\mathrm{H}}_{\mathrm{Ar}}\right), 7.42-7.34\left(\mathrm{~m}, 3 \mathrm{H}, \underline{\mathrm{H}}_{\mathrm{Ar}}\right), 7.23-7.17\left(\mathrm{~m}, 1 \mathrm{H}, \underline{\mathrm{H}}_{\mathrm{Ar}}\right), 7.01(\mathrm{dd}, J=9.1,4.6 \mathrm{~Hz}, 1 \mathrm{H}$, $\left.\underline{\mathrm{H}}_{\mathrm{Ar}}\right), 3.89\left(\mathrm{~s}, 3 \mathrm{H}, \mathrm{NC}_{3}\right)$.

${ }^{19}$ F NMR $\left(376 \mathrm{MHz}, \mathrm{CDCl}_{3}\right) \delta-124.7$.

${ }^{13} \mathrm{C}$ NMR $\left(101 \mathrm{MHz}, \mathrm{CDCl}_{3}\right) \delta 192.2(\mathrm{~d}, J=2.2 \mathrm{~Hz}), 158.0(\mathrm{~d}, J=1.5 \mathrm{~Hz}), 155.0(\mathrm{~d}, J=237.5 \mathrm{~Hz}), 137.5$, 136.9, 127.2, 124.1, 123.1, 122.5, 121.7 (d, $J=23.4 \mathrm{~Hz}), 121.1$ (d, $J=6.1 \mathrm{~Hz}), 119.3$ (d, $J=7.3 \mathrm{~Hz}), 116.7$ $(\mathrm{d}, J=23.7 \mathrm{~Hz}), 114.4,110.0,33.9$.

IR $v_{\max } 2927$ (w), 1571 (m), 1525 (s), 1464 (s), 1423 (m), 1341 (s), 1222 (s), 1125 (s), 1099 (s), 829 (s), 791 (s), $750(\mathrm{~s}), 676(\mathrm{~s})$.

HRMS calculated for $\mathrm{C}_{16} \mathrm{H}_{13} \mathrm{FNO}_{2}{ }^{+}[\mathrm{M}+\mathrm{H}]^{+}$270.0925; found 270.0930 .

\section{(5-Chloro-2-hydroxyphenyl)(1-methyl-1H-indol-3-yl)methanone (6e)}<smiles>Cn1cc(C(=O)c2cc(Cl)ccc2O)c2ccccc21</smiles>

Following General Procedure E1, starting from commercially available 5-chloro-2hydroxybenzaldehyde and Me-indoleBX 5a, a purification by column chromatography on silica gel (Pentane/EtOAc 85:15) afford the title compound 6e (39.3 mg, $0.138 \mathrm{mmol}, 92 \%$ yield) as a yellow solid. $\mathbf{m p ~} 187-189{ }^{\circ} \mathrm{C}$. Rf 0.58 (Pentane/EtOAc 4:1).

${ }^{1} \mathbf{H}$ NMR $\left(400 \mathrm{MHz}, \mathrm{CDCl}_{3}\right) \delta 11.90(\mathrm{~s}, 1 \mathrm{H}, \mathrm{OH}), 8.29\left(\mathrm{dd}, J=5.9,2.4 \mathrm{~Hz}, 1 \mathrm{H}, \underline{\mathrm{H}}_{\mathrm{Ar}}\right), 7.82(\mathrm{~d}, J=2.5 \mathrm{~Hz}$, $\left.1 \mathrm{H}, \underline{\mathrm{H}}_{\mathrm{Ar}}\right), 7.66(\mathrm{~s}, 1 \mathrm{H}, \mathrm{C} \underline{\mathrm{H}}-\mathrm{N}), 7.43-7.34\left(\mathrm{~m}, 4 \mathrm{H}, \underline{\mathrm{H}}_{\mathrm{Ar}}\right), 7.01\left(\mathrm{~d}, J=8.8 \mathrm{~Hz}, 1 \mathrm{H}, \underline{\mathrm{H}}_{\mathrm{Ar}}\right), 3.92\left(\mathrm{~s}, 3 \mathrm{H}, \mathrm{NC} \underline{\mathrm{H}}_{3}\right)$.

${ }^{13} \mathrm{C}$ NMR $\left(101 \mathrm{MHz}, \mathrm{CDCl}_{3}\right) \delta 192.1,160.5,137.6,137.0,134.4,130.5,127.3,124.2,123.4,123.2,122.5$, $122.2,119.8,114.5,110.1,33.9$.

IR $v_{\max } 1563(\mathrm{~m}), 1521$ (s), 1470 (s), 1335 (s), 1276 (s), 1217 (s), 1128 (m), 812 (s), 748 (s), 723 (s).

HRMS calculated for $\mathrm{C}_{16} \mathrm{H}_{13} \mathrm{ClNO}_{2}{ }^{+}[\mathrm{M}+\mathrm{H}]^{+}$286.0629; found 286.0636 .

\section{(5-Bromo-2-hydroxyphenyl)(1-methyl-1H-indol-3-yl)methanone (6f)}<smiles>Cn1cc(C(=O)c2cc(Br)ccc2O)c2ccccc21</smiles>

${ }^{1} \mathbf{H}$ NMR $\left(400 \mathrm{MHz}, \mathrm{CDCl}_{3}\right) \delta 11.93(\mathrm{~s}, 1 \mathrm{H}, \mathrm{OH}), 8.32-8.25\left(\mathrm{~m}, 1 \mathrm{H}, \underline{\mathrm{H}}_{\mathrm{Ar}}\right), 7.96\left(\mathrm{~d}, J=2.5 \mathrm{~Hz}, 1 \mathrm{H}, \underline{\mathrm{H}}_{\mathrm{Ar}}\right)$, $7.65(\mathrm{~s}, 1 \mathrm{H}, \mathrm{C} \underline{\mathrm{H}}-\mathrm{N}), 7.54\left(\mathrm{dd}, J=8.8,2.5 \mathrm{~Hz}, 1 \mathrm{H}, \underline{\mathrm{H}}_{\mathrm{Ar}}\right), 7.43-7.34\left(\mathrm{~m}, 3 \mathrm{H}, \underline{\mathrm{H}}_{\mathrm{Ar}}\right), 6.96(\mathrm{~d}, J=8.8 \mathrm{~Hz}, 1 \mathrm{H}$, $\left.\underline{\mathrm{H}}_{\mathrm{Ar}}\right), 3.91\left(\mathrm{~s}, 3 \mathrm{H}, \mathrm{NC} \underline{\mathrm{H}}_{3}\right)$.

${ }^{13} \mathrm{C}$ NMR $\left(101 \mathrm{MHz}, \mathrm{CDCl}_{3}\right) \delta 192.0,160.9,137.6,137.2,137.0,133.5,127.2,124.2,123.2,122.8,122.5$, $120.2,114.4,110.4,110.1,34.0$.

IR $v_{\max } 2913$ (w), 1565 (m), 1523 (s), 1463 (s), 1337 (s), 1274 (m), 1215 (s), 812 (m), 746 (s).

HRMS calculated for $\mathrm{C}_{16} \mathrm{H}_{13}{ }^{79} \mathrm{BrNO}_{2}{ }^{+}[\mathrm{M}+\mathrm{H}]^{+} 330.0124$; found 330.0129. 


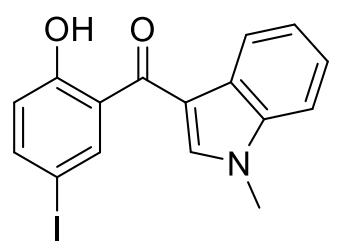

Following General Procedure E1, starting from commercially available 5-iodo-2hydroxybenzaldehyde and Me-indoleBX 5a, a purification by column chromatography on silica gel (Pentane/EtOAc 85:15) afford the title compound $\mathbf{6 g}$ (50.1 mg, $0.133 \mathrm{mmol}, 89 \%$ yield) as a yellow solid. mp $181-183{ }^{\circ} \mathrm{C}$. Rf 0.61 (Pentane/EtOAc 4:1).

${ }^{1} \mathbf{H}$ NMR $\left(400 \mathrm{MHz}, \mathrm{CDCl}_{3}\right) \delta 11.94(\mathrm{~s}, 1 \mathrm{H}, \mathrm{O} \underline{\mathrm{H}}), 8.29-8.25\left(\mathrm{~m}, 1 \mathrm{H}, \underline{\mathrm{H}}_{\mathrm{Ar}}\right), 8.13\left(\mathrm{~d}, J=2.2 \mathrm{~Hz}, 1 \mathrm{H}, \underline{\mathrm{H}}_{\mathrm{Ar}}\right)$, $7.70\left(\mathrm{dd}, J=8.7,2.2 \mathrm{~Hz}, 1 \mathrm{H}, \underline{\mathrm{H}}_{\mathrm{Ar}}\right), 7.63(\mathrm{~s}, 1 \mathrm{H}, \mathrm{C} \underline{\mathrm{H}}-\mathrm{N}), 7.43-7.34\left(\mathrm{~m}, 3 \mathrm{H}, \underline{\mathrm{H}}_{\mathrm{Ar}}\right), 6.85(\mathrm{~d}, J=8.7 \mathrm{~Hz}, 1 \mathrm{H}$, $\left.\underline{\mathrm{H}}_{\mathrm{Ar}}\right), 3.92\left(\mathrm{~s}, 3 \mathrm{H}, \mathrm{NC}_{3}\right)$.

${ }^{13} \mathrm{C}$ NMR $\left(101 \mathrm{MHz}, \mathrm{CDCl}_{3}\right) \delta 191.9,161.6,142.9,139.5,137.6,137.0,127.2,124.2,123.7,123.2,122.5$, $120.7,114.5,110.1,79.7,34.0$.

IR $v_{\max } 1570$ (s), 1554 (s), 1520 (s), 1461 (s), 1337 (s), 1272 (s), 1213 (s), 1108 (s), 888 (s), 808 (s), 755 (s), 736 (s).

HRMS calculated for $\mathrm{C}_{16} \mathrm{H}_{13} \mathrm{INO}_{2}{ }^{+}[\mathrm{M}+\mathrm{H}]^{+}$377.9986; found 377.9984 .

\section{4-Hydroxy-3-(1-methyl-1H-indole-3-carbonyl)benzaldehyde (6h)}<smiles>Cn1cc(C(=O)c2cc(C=O)ccc2O)c2ccccc21</smiles>

Following General Procedure E1, starting from commercially available 4hydroxyisophthalaldehyde and Me-indoleBX 5a, a purification by column chromatography on silica gel (Pentane/EtOAc 3:2) afford the title compound $\mathbf{6 h}$ (34.3 mg, $0.123 \mathrm{mmol}, 82 \%$ yield) as a yellow solid. $\mathbf{m p} 203-205{ }^{\circ} \mathrm{C}$. Rf 0.50 (Pentane/EtOAc 1:1).

${ }^{1} \mathbf{H}$ NMR $\left(400 \mathrm{MHz}, \mathrm{CDCl}_{3}\right) \delta 12.84(\mathrm{~s}, 1 \mathrm{H}, \mathrm{OH}), 9.90(\mathrm{~s}, 1 \mathrm{H}, \mathrm{C} \underline{\mathrm{HO}}), 8.43\left(\mathrm{~d}, J=2.0 \mathrm{~Hz}, 1 \mathrm{H}, \underline{\mathrm{H}}_{\mathrm{Ar}}\right), 8.32-$ $8.27\left(\mathrm{~m}, 1 \mathrm{H}, \underline{\mathrm{H}}_{\mathrm{Ar}}\right), 7.98\left(\mathrm{dd}, J=8.6,2.1 \mathrm{~Hz}, 1 \mathrm{H}, \underline{\mathrm{H}}_{\mathrm{Ar}}\right), 7.71(\mathrm{~s}, 1 \mathrm{H}, \underline{\mathrm{C}} \underline{\mathrm{H}}-\mathrm{N}), 7.44-7.35\left(\mathrm{~m}, 3 \mathrm{H}, \underline{\mathrm{H}}_{\mathrm{Ar}}\right), 7.17(\mathrm{~d}$, $\left.J=8.6 \mathrm{~Hz}, 1 \mathrm{H}, \underline{\mathrm{H}}_{\mathrm{Ar}}\right), 3.93\left(\mathrm{~s}, 3 \mathrm{H}, \mathrm{NCH}_{3}\right)$.

${ }^{13} \mathrm{C}$ NMR $\left(101 \mathrm{MHz}, \mathrm{CDCl}_{3}\right) \delta 192.6,190.3,167.5,137.6,137.2,135.9,133.6,128.1,127.3,124.4,123.3$, $122.5,121.3,119.2,114.4,110.1,34.0$.

IR $v_{\max } 3115$ (w), 2921 (m), 1693 (s), 1609 (s), 1562 (s), 1525 (s), 1469 (s), 1354 (s), 1229 (s), 1102 (s), 834 (s), 741 (s), 725 (s).

HRMS calculated for $\mathrm{C}_{17} \mathrm{H}_{14} \mathrm{NO}_{3}{ }^{+}[\mathrm{M}+\mathrm{H}]^{+}$280.0968; found 280.0966 .

(2-Hydroxy-5-nitrophenyl)(1-methyl-1H-indol-3-yl)methanone (6i)<smiles>Cn1cc(C(=O)c2cc([N+](=O)[O-])ccc2O)c2ccccc21</smiles>

Following General Procedure E1, starting from commercially available 2-hydroxy5-nitrobenzaldehyde and Me-indoleBX 5a, a purification by column chromatography on silica gel (Pentane/DCM 1:1) afford the title compound $6 \mathbf{i}$ (32.9 $\mathrm{mg}, 0.111 \mathrm{mmol}, 74 \%$ yield) as a yellow solid. mp $227-229{ }^{\circ} \mathrm{C}$. Rf 0.44 (Pentane/EtOAc 4:1).

${ }^{1} \mathbf{H}$ NMR $\left(400 \mathrm{MHz}, \mathrm{CDCl}_{3}\right) \delta 12.97(\mathrm{~s}, 1 \mathrm{H}, \mathrm{OH}), 8.86\left(\mathrm{~d}, J=2.7 \mathrm{~Hz}, 1 \mathrm{H}, \underline{\mathrm{H}}_{\mathrm{Ar}}\right), 8.35(\mathrm{dd}, J=9.2,2.7 \mathrm{~Hz}$, $\left.1 \mathrm{H}, \underline{\mathrm{H}}_{\mathrm{Ar}}\right), 8.33-8.29\left(\mathrm{~m}, 1 \mathrm{H}, \underline{\mathrm{H}}_{\mathrm{Ar}}\right), 7.75(\mathrm{~s}, 1 \mathrm{H}, \mathrm{C} \underline{\mathrm{H}}-\mathrm{N}), 7.47-7.37\left(\mathrm{~m}, 3 \mathrm{H}, \underline{\mathrm{H}}_{\mathrm{Ar}}\right), 7.14\left(\mathrm{~d}, J=9.2 \mathrm{~Hz}, 1 \mathrm{H}, \underline{\mathrm{H}}_{\mathrm{Ar}}\right)$, $3.96\left(\mathrm{~s}, 3 \mathrm{H}, \mathrm{NC}_{3}\right)$.

${ }^{13}$ C NMR $\left(101 \mathrm{MHz}, \mathrm{CDCl}_{3}\right) \delta 191.6,167.6,139.7,137.8,137.4,129.7,127.6,127.3,124.7,123.7,122.7$, $120.4,119.3,114.2,110.4,34.3$.

IR $v_{\max } 1581(\mathrm{~m}), 1522(\mathrm{~s}), 1458(\mathrm{~m}), 1341$ (s), 1286 (s), 1224 (s), 1095 (m), 832 (s), 735 (s), 722 (s).

HRMS calculated for $\mathrm{C}_{16} \mathrm{H}_{13} \mathrm{~N}_{2} \mathrm{O}_{4}{ }^{+}[\mathrm{M}+\mathrm{H}]^{+} 297.0870$; found 297.0874 . 
<smiles>COc1cccc(C(=O)c2cn(C)c3ccccc23)c1O</smiles>

Following General Procedure E1, starting from commercially available 2hydroxy-3-methoxybenzaldehyde and Me-indoleBX 5a, a purification by column chromatography on silica gel (Pentane/EtOAc 7:3) afford the title compound $\mathbf{6 j}$ (39.6 mg, $0.141 \mathrm{mmol}, 94 \%$ yield) as a yellow solid. mp 134$136{ }^{\circ} \mathrm{C}$. Rf 0.56 (Pentane/EtOAc 1:1).

${ }^{1} \mathbf{H}$ NMR $\left(400 \mathrm{MHz}, \mathrm{CDCl}_{3}\right) \delta 12.22(\mathrm{bs}, 1 \mathrm{H}, \mathrm{O} \underline{\mathrm{H}}), 8.32-8.28\left(\mathrm{~m}, 1 \mathrm{H}, \underline{\mathrm{H}}_{\mathrm{Ar}}\right), 7.61(\mathrm{~s}, 1 \mathrm{H}, \mathrm{C} \underline{\mathrm{H}}-\mathrm{N}), 7.42$ (dd, $\left.J=8.0,1.4 \mathrm{~Hz}, 1 \mathrm{H}, \underline{\mathrm{H}}_{\mathrm{Ar}}\right), 7.36-7.30\left(\mathrm{~m}, 3 \mathrm{H}, \underline{\mathrm{H}}_{\mathrm{Ar}}\right), 7.05\left(\mathrm{dd}, J=8.0,1.3 \mathrm{~Hz}, 1 \mathrm{H}, \underline{\mathrm{H}}_{\mathrm{Ar}}\right), 6.86(\mathrm{t}, J=8.0 \mathrm{~Hz}$, $\left.1 \mathrm{H}, \underline{\mathrm{H}}_{\mathrm{Ar}}\right), 3.92\left(\mathrm{~s}, 3 \mathrm{H}, \mathrm{OC}_{\mathrm{H}}\right), 3.84\left(\mathrm{~s}, 3 \mathrm{H}, \mathrm{NC}_{3}\right)$.

${ }^{13}$ C NMR $\left(101 \mathrm{MHz}, \mathrm{CDCl}_{3}\right) \delta 193.5,152.0,148.9,137.4,137.3,127.3,123.9,122.9,122.8,122.5,121.6$, $118.1,115.6,114.8,109.9,56.3,33.7$.

IR $v_{\max } 2933$ (w), 1563 (s), 1524 (s), 1461 (s), 1350 (s), 1239 (s), 1088 (m), 931 (m), 743 (s).

HRMS calculated for $\mathrm{C}_{17} \mathrm{H}_{16} \mathrm{NO}_{3}{ }^{+}[\mathrm{M}+\mathrm{H}]^{+} 282.1125$; found 282.1132 .

\section{(2-Hydroxy-4-methoxyphenyl)(1-methyl-1H-indol-3-yl)methanone (6k)}<smiles>COc1ccc(C(=O)c2cn(C)c3ccccc23)c(O)c1</smiles>

Following General Procedure E1, starting from commercially available 2hydroxy-4-methoxybenzaldehyde and Me-indoleBX 5a, a purification by column chromatography on silica gel (Pentane/EtOAc 4:1) afford the title compound $\mathbf{6 k}(39.2 \mathrm{mg}, 0.140 \mathrm{mmol}, 93 \%$ yield $)$ as a yellow solid. $\mathbf{m p} 133-$ $135^{\circ} \mathrm{C}$. Rf 0.40 (Pentane/EtOAc 4:1).

${ }^{1} \mathbf{H}$ NMR $\left(400 \mathrm{MHz}, \mathrm{CDCl}_{3}\right) \delta 12.88(\mathrm{~s}, 1 \mathrm{H}, \mathrm{OH}), 8.24-8.20\left(\mathrm{~m}, 1 \mathrm{H}, \underline{\mathrm{H}}_{\mathrm{Ar}}\right), 7.79\left(\mathrm{~d}, J=8.8 \mathrm{~Hz}, 1 \mathrm{H}, \underline{\mathrm{H}}_{\mathrm{Ar}}\right)$, $7.58(\mathrm{~s}, 1 \mathrm{H}, \mathrm{C} \underline{\mathrm{H}}-\mathrm{N}), 7.38-7.29\left(\mathrm{~m}, 3 \mathrm{H}, \underline{\mathrm{H}}_{\mathrm{Ar}}\right), 6.52\left(\mathrm{~d}, J=2.5 \mathrm{~Hz}, 1 \mathrm{H}, \underline{\mathrm{H}}_{\mathrm{Ar}}\right), 6.45(\mathrm{dd}, J=8.9,2.5 \mathrm{~Hz}, 1 \mathrm{H}$, $\left.\underline{\mathrm{H}}_{\mathrm{Ar}}\right), 3.85$ (s, $\left.6 \mathrm{H}, \mathrm{OC}_{3}+\mathrm{NC}_{3}\right)$.

${ }^{13} \mathrm{C} \mathrm{NMR}\left(101 \mathrm{MHz}, \mathrm{CDCl}_{3}\right) \delta$ 192.7, 165.2, 165.1, 137.3, 135.8, 133.3, 127.3, 123.6, 122.5, 122.3, 114.9, 114.7, 109.9, 106.8, 101.3, 55.6, 33.6.

IR $v_{\max } 2920$ (w), 2839 (w), 1618 (s), 1560 (s), 1505 (s), 1459 (s), 1369 (s), 1340 (s), 1299 (s), 1267 (s), 1241 (s), 1221 (s), 1145 (s), 1107 (s), 852 (s), 751 (s).

HRMS calculated for $\mathrm{C}_{17} \mathrm{H}_{16} \mathrm{NO}_{3}{ }^{+}[\mathrm{M}+\mathrm{H}]^{+}$282.1125; found 282.1127 .

\section{(2-Hydroxy-6-methoxyphenyl)(1-methyl-1H-indol-3-yl)methanone (6l)}<smiles>COc1cccc(O)c1C(=O)c1cn(C)c2ccccc12</smiles>

Following General Procedure E1 (30 minutes), starting from 2-hydroxy-6methoxybenzaldehyde $\mathbf{1 3}$ and Me-indoleBX 5a, a purification by column chromatography on silica gel (Pentane/EtOAc 7:3) afford the title compound $\mathbf{6 1}$ (36.1 mg, $0.128 \mathrm{mmol}, 86 \%$ yield) as a off-white solid. $\mathbf{m p} 250-252{ }^{\circ} \mathrm{C}$. Rf 0.54 (Pentane/EtOAc 1:1).

${ }^{1}$ H NMR (400 MHz, DMSO-d6) $\delta 9.49(\mathrm{~s}, 1 \mathrm{H}, \mathrm{OH}), 8.09$ (d, $\left.J=7.6 \mathrm{~Hz}, 1 \mathrm{H}, \underline{\mathrm{H}}_{\mathrm{Ar}}\right), 7.60$ (s, 1H, C$-\mathrm{H}$ ), $7.52\left(\mathrm{~d}, J=8.0 \mathrm{~Hz}, 1 \mathrm{H}, \underline{\mathrm{H}}_{\mathrm{Ar}}\right), 7.26\left(\mathrm{dtd}, J=21.1,7.2,1.2 \mathrm{~Hz}, 2 \mathrm{H}, \underline{\mathrm{H}}_{\mathrm{Ar}}\right), 7.18\left(\mathrm{t}, J=8.3 \mathrm{~Hz}, 1 \mathrm{H}, \underline{\mathrm{H}}_{\mathrm{Ar}}\right), 6.55$ (dd, $\left.J=8.2,4.3 \mathrm{~Hz}, 2 \mathrm{H}, \underline{\mathrm{H}}_{\mathrm{Ar}}\right), 3.80\left(\mathrm{~s}, 3 \mathrm{H}, \mathrm{NC}_{3}\right), 3.63$ (s, $\left.3 \mathrm{H}, \mathrm{OC}_{3}\right)$.

${ }^{13}$ C NMR (101 MHz, DMSO-d6) $\delta$ 188.0, 157.2, 154.9, 138.9, 137.5, 129.6, 125.7, 122.8, 122.1, 121.2, 188.6, 116.9, 110.7, 108.7, 102.1, 55.4, 33.0.

IR $v_{\max } 3150$ (br), 1596 (s), 1573 (s), 1527 (s), 1459 (s), 1371 (s), 1227 (s), 1078 (s), 876 (s), 757 (s), 741 (s), $712(\mathrm{~s})$.

HRMS calculated for $\mathrm{C}_{17} \mathrm{H}_{16} \mathrm{NO}_{3}{ }^{+}[\mathrm{M}+\mathrm{H}]^{+}$282.1125; found 282.1124 . 
<smiles>Cc1cccc(C(=O)c2cn(C)c3ccccc23)c1O</smiles>

Following General Procedure E1, starting from commercially available 2hydroxy-3-methylbenzaldehyde and Me-indoleBX 5a, a purification by column chromatography on silica gel (Pentane/EtOAc 85:15) afforded the title compound $\mathbf{6 m}(34.9 \mathrm{mg}, 0.132 \mathrm{mmol}, 88 \%$ yield) as a yellow solid. $\mathbf{m p} 110-112$ ${ }^{\circ} \mathrm{C}$. Rf 0.45 (Pentane/EtOAc 4:1).

${ }^{1} \mathbf{H}$ NMR $\left(400 \mathrm{MHz}, \mathrm{CDCl}_{3}\right) \delta 12.31(\mathrm{bs}, 1 \mathrm{H}, \mathrm{O} \underline{\mathrm{H}}), 8.31-8.28\left(\mathrm{~m}, 1 \mathrm{H}, \underline{\mathrm{H}}_{\mathrm{Ar}}\right), 7.70(\mathrm{dd}, J=7.9,1.2 \mathrm{~Hz}, 1 \mathrm{H}$, $\left.\underline{\mathrm{H}}_{\mathrm{Ar}}\right), 7.61(\mathrm{~s}, 1 \mathrm{H}, \mathrm{CH}-\mathrm{N}), 7.39-7.32\left(\mathrm{~m}, 4 \mathrm{H}, \underline{\mathrm{H}}_{\mathrm{Ar}}\right), 6.83\left(\mathrm{t}, J=7.6 \mathrm{~Hz}, 1 \mathrm{H}, \underline{\mathrm{H}}_{\mathrm{Ar}}\right), 3.86\left(\mathrm{~s}, 3 \mathrm{H}, \mathrm{NC} \underline{\mathrm{H}}_{3}\right), 2.34(\mathrm{~s}$, $\left.3 \mathrm{H}, \mathrm{C}_{3}\right)$.

${ }^{13}$ C NMR $\left(101 \mathrm{MHz}, \mathrm{CDCl}_{3}\right) \delta 194.0,160.4,137.4,136.9,135.6,129.2,127.4,127.1,123.8,122.7,122.5$, $120.7,118.0,114.9,109.9,33.7,15.9$.

IR $v_{\max } 2906(\mathrm{w}), 1599(\mathrm{~m}), 1562$ (m), 1517 (s), 1471 (m), 1425 (m), 1339 (s), 1238 (s), 1078 (s), 741 (s).

HRMS calculated for $\mathrm{C}_{17} \mathrm{H}_{16} \mathrm{NO}_{2}{ }^{+}[\mathrm{M}+\mathrm{H}]^{+}$266.1176; found 266.1179.

\section{(3,5-Di-tert-butyl-2-hydroxyphenyl)(1-methyl-1H-indol-3-yl)methanone (6n)}

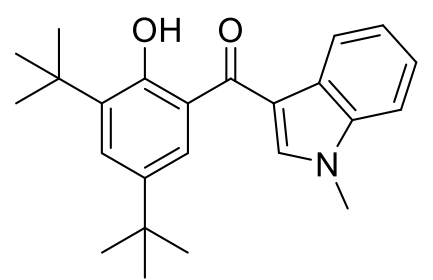

Following General Procedure E1 (60 minutes), starting from commercially available 3,5-di-tert-butyl-2-hydroxybenzaldehyde and Me-indoleBX 5a, a purification by column chromatography on silica gel (Pentane/ $\mathrm{Et}_{2} \mathrm{O}$ 9:1) afforded the title compound $\mathbf{6 n}(30.8 \mathrm{mg}, 0.085 \mathrm{mmol}, 85 \%$ yield $)$ as a yellow solid. mp 210-212 ${ }^{\circ} \mathrm{C}$. Rf 0.78 (Pentane/EtOAc 4:1).

${ }^{1} \mathbf{H}$ NMR $\left(400 \mathrm{MHz}, \mathrm{CDCl}_{3}\right) \delta 12.49(\mathrm{~s}, 1 \mathrm{H}, \mathrm{OH}), 8.24(\mathrm{dd}, J=6.8,1.4 \mathrm{~Hz}$, $\left.1 \mathrm{H}, \underline{\mathrm{H}}_{\mathrm{Ar}}\right), 7.73\left(\mathrm{~d}, J=2.5 \mathrm{~Hz}, 1 \mathrm{H}, \underline{\mathrm{H}}_{\mathrm{Ar}}\right), 7.61(\mathrm{~s}, 1 \mathrm{H}, \mathrm{C} \underline{\mathrm{H}}-\mathrm{N}), 7.56\left(\mathrm{~d}, J=2.4 \mathrm{~Hz}, 1 \mathrm{H}, \underline{\mathrm{H}}_{\mathrm{Ar}}\right), 7.42-7.31(\mathrm{~m}, 3 \mathrm{H}$, $\left.\underline{\mathrm{H}}_{\mathrm{Ar}}\right), 3.89$ (s, 3H, $\left.\mathrm{NCH}_{3}\right), 1.50\left(\mathrm{~s}, 9 \mathrm{H}, \mathrm{C}\left(\mathrm{CH}_{3}\right)_{3}\right), 1.32$ (s, 9H, $\left.\mathrm{C}\left(\mathrm{C}_{3}\right)_{3}\right)$.

${ }^{13} \mathrm{C}$ NMR $\left(101 \mathrm{MHz}, \mathrm{CDCl}_{3}\right) \delta 194.8,159.0,139.5,137.4,137.3,136.5,129.3,127.3,126.2,123.6,122.5$ (2C), 120.5, 115.5, 109.8, 35.2, 34.3, 33.6, 31.5, 29.5.

IR $v_{\max } 2947$ (m), 2866 (m), 1567 (s), 1518 (s), 1459 (s), 1429 (s), 1361 (s), 1217 (s), 1125 (s), 1078 (s), 799 (s), 750 (s), 731 (s).

HRMS calculated for $\mathrm{C}_{24} \mathrm{H}_{30} \mathrm{NO}_{2}{ }^{+}[\mathrm{M}+\mathrm{H}]^{+}$364.2271; found 364.2280.

\section{(2-Hydroxynaphthalen-1-yl)(1-methyl-1H-indol-3-yl)methanone (6o)}

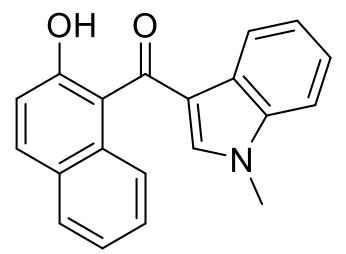

Following General Procedure E1, starting from commercially available 2-hydroxy1-naphthaldehyde and Me-indoleBX 5a, a purification by column chromatography on silica gel (Pentane/EtOAc 7:3) afforded the title compound $\mathbf{6 0}$ (44.1 mg, 0.136 mmol, $91 \%$ yield) as a white solid. $\mathbf{m p} 261-263{ }^{\circ} \mathrm{C}$. Rf 0.65 (Pentane/EtOAc 1:1).

1H NMR (400 MHz, DMSO-d6) $\delta 9.84(\mathrm{~s}, 1 \mathrm{H}, \mathrm{OH}), 8.21$ (bs, 1H, $\left.\underline{\mathrm{H}}_{\mathrm{Ar}}\right), 7.87$ (t, $J$ $\left.=8.1 \mathrm{~Hz}, 2 \mathrm{H}, \underline{\mathrm{H}}_{\mathrm{Ar}}\right), 7.59(\mathrm{~s}, 1 \mathrm{H}, \mathrm{C} \underline{\mathrm{H}}-\mathrm{N}), 7.54\left(\mathrm{~d}, J=7.4 \mathrm{~Hz}, 1 \mathrm{H}, \underline{\mathrm{H}}_{\mathrm{Ar}}\right), 7.45\left(\mathrm{~d}, J=8.2 \mathrm{~Hz}, 1 \mathrm{H}, \underline{\mathrm{H}}_{\mathrm{Ar}}\right), 7.36-$ $7.26\left(\mathrm{~m}, 5 \mathrm{H}, \underline{\mathrm{H}}_{\mathrm{Ar}}\right), 3.76\left(\mathrm{~s}, 3 \mathrm{H}, \mathrm{NC}_{\underline{H}_{3}}\right)$.

13C NMR (101 MHz, DMSO-d6) $\delta$ 190.4, 151.3, 139.6, 137.6, 131.9, 129.7, 128.0, 127.5, 126.6, 125.8, $123.6,123.0,122.8,122.3,122.0,121.3,118.5,117.0,110.8,33.0$.

IR $v_{\max } 3109$ (br), 1594 (s), 1524 (s), 1459 (m), 1368 (m), 1347 (m), 1242 (m), 1212 (s), 1090 (m), 819 (m), 754 (s).

HRMS calculated for $\mathrm{C}_{20} \mathrm{H}_{15} \mathrm{NNaO}_{2}{ }^{+}[\mathrm{M}+\mathrm{Na}]^{+}$324.0995; found 324.0991. 
$(8 R, 9 S, 13 S, 14 S)-3-H y d r o x y-13-m e t h y l-2-(1-m e t h y l-1 H$-indole-3-carbonyl)-7,8,9,11,12,13,15,16octahydro-6H-cyclopenta $[a]$ phenanthren-17(14H)-one $(6 p)$

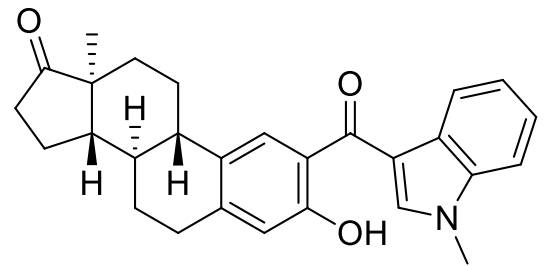

Following General Procedure E1, starting from 2-formyl estrone 13 and Me-indoleBX 5a, a purification by column chromatography on silica gel (Pentane/EtOAc 3:2) afforded the title compound $\mathbf{6 p}(63.0$ $\mathrm{mg}, 0.147 \mathrm{mmol}, 98 \%$ yield) as a yellow solid. $\mathbf{m p} 135-137^{\circ} \mathrm{C}$. Rf 0.62 (Pentane/EtOAc 1:1).

${ }^{1} \mathbf{H}$ NMR $\left(400 \mathrm{MHz}, \mathrm{CDCl}_{3}\right) \delta 11.85(\mathrm{bs}, 1 \mathrm{H}, \mathrm{OH}), 8.23\left(\mathrm{~d}, J=7.2 \mathrm{~Hz}, 1 \mathrm{H}, \underline{\mathrm{H}}_{\mathrm{Ar}}\right), 7.76\left(\mathrm{~s}, 1 \mathrm{H}, \underline{\mathrm{H}}_{\mathrm{Ar}}\right), 7.61$ (s, 1H, C$-\mathrm{H}-\mathrm{N}), 7.40-7.30\left(\mathrm{~m}, 3 \mathrm{H}, \underline{\mathrm{H}}_{\mathrm{Ar}}\right), 6.78\left(\mathrm{~s}, 1 \mathrm{H}, \underline{\mathrm{H}}_{\mathrm{Ar}}\right), 3.90$ (s, 3H, NC$\left.\underline{\mathrm{H}}_{3}\right), 3.00-2.92\left(\mathrm{~m}, 2 \mathrm{H}, \underline{\mathrm{H}}_{\mathrm{aliph}}\right)$, $2.52\left(\mathrm{dd}, J=18.6,8.7 \mathrm{~Hz}, 1 \mathrm{H}, \underline{\mathrm{H}}_{\mathrm{aliph}}\right), 2.32-2.22\left(\mathrm{~m}, 2 \mathrm{H}, \underline{\mathrm{H}}_{\mathrm{aliph}}\right), 2.20-2.00$ (m, 3H, $\left.\underline{\mathrm{H}}_{\mathrm{aliph}}\right), 1.96-1.89$ (m, $\left.1 \mathrm{H}, \underline{\mathrm{H}}_{\text {aliph }}\right), 1.70-1.40$ (m, $\left.6 \mathrm{H}, \underline{\mathrm{H}}_{\text {aliph }}\right), 0.93$ (s, 3H, $\left.\underline{\mathrm{C}}_{3}\right)$.

${ }^{13} \mathrm{C}$ NMR $\left(101 \mathrm{MHz}, \mathrm{CDCl}_{3}\right) \delta 220.8,193.6,159.8,144.8,137.5,136.2,130.4,128.6,127.3,123.9$, 122.7, 122.5, 119.5, 117.6, 115.1, 109.9, 50.5, 48.1, 43.9, 38.4, 36.0, 33.9, 31.6, 29.9, 26.4, 26.1, 21.7, 14.0.

IR $v_{\max } 2927$ (m), 2852 (w), 1736 (s), 1566 (m), 1521 (s), 1463 (s), 1363 (s), 1221 (s), 847 (s), 737 (s).

HRMS calculated for $\mathrm{C}_{28} \mathrm{H}_{29} \mathrm{NNaO}_{3}{ }^{+}[\mathrm{M}+\mathrm{Na}]^{+} 450.2040$; found 450.2041.

\section{(4-Hydroxy-9H-carbazol-3-yl)(1-methyl-1H-indol-3-yl)methanone (6q)}<smiles>Cn1cc(C(=O)c2ccc3[nH]c4ccccc4c3c2O)c2ccccc21</smiles>

Following General Procedure E1, starting from 4-hydroxy-9H-carbazole3-carbaldehyde $\mathbf{4 q}$ and Me-indoleBX 5a, a purification by column chromatography on silica gel (Pentane/EtOAc 1:1) afford the title compound 6q (45.9 mg, $0.135 \mathrm{mmol}, 90 \%$ yield) as a ochre solid. mp 238$240{ }^{\circ} \mathrm{C}$. Rf 0.36 (Pentane/EtOAc $1: 1$ ).

1H NMR $\left(400 \mathrm{MHz}, \mathrm{CDCl}_{3}\right) \delta 13.84(\mathrm{~s}, 1 \mathrm{H}, \mathrm{O} \underline{\mathrm{H}}), 8.46\left(\mathrm{~d}, J=7.7 \mathrm{~Hz}, 1 \mathrm{H}, \underline{\mathrm{H}}_{\mathrm{Ar}}\right), 8.28-8.23(\mathrm{~m}, 2 \mathrm{H}, \mathrm{N} \underline{\mathrm{H}}+$ $\left.\underline{\mathrm{H}}_{\mathrm{Ar}}\right), 7.93\left(\mathrm{~d}, J=8.6 \mathrm{~Hz}, 1 \mathrm{H}, \underline{\mathrm{H}}_{\mathrm{Ar}}\right), 7.65(\mathrm{~s}, 1 \mathrm{H}, \mathrm{C} \underline{\mathrm{H}}-\mathrm{N}), 7.46-7.29\left(\mathrm{~m}, 6 \mathrm{H}, \underline{\mathrm{H}}_{\mathrm{Ar}}\right), 6.92(\mathrm{~d}, J=8.6 \mathrm{~Hz}, 1 \mathrm{H}$, $\left.\underline{\mathrm{H}}_{\mathrm{Ar}}\right), 3.89$ ( $\left.\mathrm{s}, 3 \mathrm{H}, \mathrm{NC} \underline{\mathrm{H}}_{3}\right)$.

${ }^{13} \mathrm{C}$ NMR $\left(101 \mathrm{MHz}, \mathrm{CDCl}_{3}\right) \delta 193.8,161.4,144.3,138.8,137.4,135.7,130.2,127.5,125.5,123.6,123.5$, $123.4,122.5,122.4,121.0,115.3,113.2,112.1,110.4,109.9,101.9,33.7$.

IR $v_{\max } 3672$ (w), 2982 (s), 2902 (s), 1590 (m), 1524 (m), 1459 (m), 1393 (s), 1382 (s), 1368 (s), 1263 (s), 1240 (s), 1230 (s), 1075 (s), 1045 (s), 740 (s), 720 (s).

HRMS calculated for $\mathrm{C}_{22} \mathrm{H}_{17} \mathrm{~N}_{2} \mathrm{O}_{2}{ }^{+}[\mathrm{M}+\mathrm{H}]^{+} 341.1285$; found 341.1288 .

\section{(2-Hydroxyphenyl)(1-pentyl-1H-indol-3-yl)methanone (6r)}

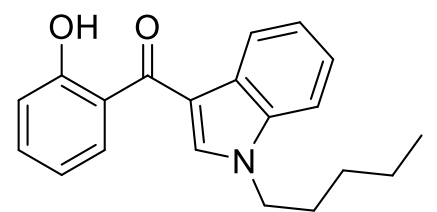

Following General Procedure E1, starting from commercially available salicylaldehyde and Pe-indoleBX $\mathbf{5 b}$, a purification by column chromatography on silica gel (Pentane/EtOAc 19:1) afford the title compound 6r (42.5 mg, $0.138 \mathrm{mmol}, 92 \%$ yield) as a yellow solid. $\mathbf{m p} 76-78^{\circ} \mathrm{C} . \mathbf{R f} 0.85$ (Pentane/EtOAc 4:1).

${ }^{1} \mathbf{H}$ NMR $\left(400 \mathrm{MHz}, \mathrm{CDCl}_{3}\right) \delta 12.07$ (bs, $\left.1 \mathrm{H}, \mathrm{OH}\right), 8.34-8.29\left(\mathrm{~m}, 1 \mathrm{H}, \underline{\mathrm{H}}_{\mathrm{Ar}}\right), 7.86(\mathrm{dd}, J=7.9,1.6 \mathrm{~Hz}, 1 \mathrm{H}$, $\left.\underline{\mathrm{H}}_{\mathrm{Ar}}\right), 7.68(\mathrm{~s}, 1 \mathrm{H}, \mathrm{C} \underline{\mathrm{H}}-\mathrm{N}), 7.48\left(\mathrm{ddd}, J=8.8,7.3,1.7 \mathrm{~Hz}, 1 \mathrm{H}, \underline{\mathrm{H}}_{\mathrm{Ar}}\right), 7.44-7.40\left(\mathrm{~m}, 1 \mathrm{H}, \underline{\mathrm{H}}_{\mathrm{Ar}}\right), 7.38-7.32(\mathrm{~m}$, $\left.2 \mathrm{H}, \underline{\mathrm{H}}_{\mathrm{Ar}}\right), 7.07\left(\mathrm{~d}, J=8.3 \mathrm{~Hz}, 1 \mathrm{H}, \underline{\mathrm{H}}_{\mathrm{Ar}}\right), 6.94\left(\mathrm{t}, J=7.6 \mathrm{~Hz}, 1 \mathrm{H}, \underline{\mathrm{H}}_{\mathrm{Ar}}\right), 4.19\left(\mathrm{t}, J=7.2 \mathrm{~Hz}, 2 \mathrm{H}, \mathrm{NC}_{2}\right), 1.90$ (p, $\left.J=7.3 \mathrm{~Hz}, 2 \mathrm{H}, \underline{\mathrm{H}}_{\mathrm{aliph}}\right), 1.43-1.30\left(\mathrm{~m}, 4 \mathrm{H}, \underline{\mathrm{H}}_{\mathrm{aliph}}\right), 0.91\left(\mathrm{t}, J=6.9 \mathrm{~Hz}, 3 \mathrm{H}, \mathrm{C}_{3}\right)$.

${ }^{13} \mathrm{C}$ NMR $\left(101 \mathrm{MHz}, \mathrm{CDCl}_{3}\right) \delta 193.6,162.0,136.8,136.1,134.6,131.5,127.5,123.8,122.8,122.6,121.5$, 118.7, 118.2, 114.7, 110.2, 47.4, 29.7, 29.1, 22.3, 14.0.

IR $v_{\max } 2927(\mathrm{~m}), 2859(\mathrm{~m}), 1564(\mathrm{~m}), 1517$ (s), 1482 (s), 1463 (s), 1388 (s), 1347 (m), 1216 (m), 1194 (s), $1131(\mathrm{~s}), 887(\mathrm{~m}), 765(\mathrm{~s}), 736(\mathrm{~s}), 671(\mathrm{~s})$.

HRMS calculated for $\mathrm{C}_{20} \mathrm{H}_{22} \mathrm{NO}_{2}{ }^{+}[\mathrm{M}+\mathrm{H}]^{+} 308.1645$; found 308.1648. 


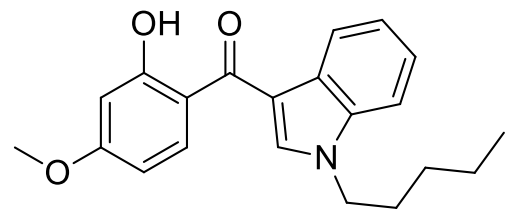

Following General Procedure E1, starting from commercially available 2-hydroxy-4-methoxybenzaldehyde and Pe-indoleBX 5b, a purification by column chromatography on silica gel (Pentane/EtOAc 9:1) afforded the title compound $\mathbf{6 s}$ (45.0 $\mathrm{mg}, 0.133 \mathrm{mmol}, 89 \%$ yield) as a yellow solid. mp 87-89 ${ }^{\circ} \mathrm{C}$. Rf 0.75 (Pentane/EtOAc 4:1).

${ }^{1} \mathbf{H}$ NMR $\left(400 \mathrm{MHz}, \mathrm{CDCl}_{3}\right) \delta 12.85(\mathrm{bs}, 1 \mathrm{H}, \mathrm{O} \underline{\mathrm{H}}), 8.25-8.21\left(\mathrm{~m}, 1 \mathrm{H}, \underline{\mathrm{H}}_{\mathrm{Ar}}\right), 7.80\left(\mathrm{~d}, J=8.8 \mathrm{~Hz}, 1 \mathrm{H}, \underline{\mathrm{H}}_{\mathrm{Ar}}\right)$, $7.65(\mathrm{~s}, 1 \mathrm{H}, \mathrm{CH}-\mathrm{N}), 7.43-7.38\left(\mathrm{~m}, 1 \mathrm{H}, \underline{\mathrm{H}}_{\mathrm{Ar}}\right), 7.35-7.28\left(\mathrm{~m}, 2 \mathrm{H}, \underline{\mathrm{H}}_{\mathrm{Ar}}\right), 6.54\left(\mathrm{~d}, J=2.5 \mathrm{~Hz}, 1 \mathrm{H}, \underline{\mathrm{H}}_{\mathrm{Ar}}\right), 6.47$ $\left(\mathrm{dd}, J=8.8,2.5 \mathrm{~Hz}, 1 \mathrm{H}, \underline{\mathrm{H}}_{\mathrm{Ar}}\right), 4.19\left(\mathrm{t}, J=7.2 \mathrm{~Hz}, 2 \mathrm{H}, \mathrm{NC}_{2}\right), 3.86\left(\mathrm{~s}, 3 \mathrm{H}, \mathrm{OC}_{3}\right), 1.90$ (p, $J=7.4 \mathrm{~Hz}, 2 \mathrm{H}$, $\left.\underline{\mathrm{H}}_{\text {aliph }}\right), 1.42-1.30\left(\mathrm{~m}, 4 \mathrm{H}, \underline{\mathrm{H}}_{\text {aliph }}\right), 0.91\left(\mathrm{t}, J=6.9 \mathrm{~Hz}, 3 \mathrm{H}, \mathrm{C}_{3}\right)$.

${ }^{13} \mathrm{C}$ NMR $\left(101 \mathrm{MHz}, \mathrm{CDCl}_{3}\right) \delta 192.8,165.2,165.1,136.7,134.9,133.3,127.5,123.5,122.4,122.4,115.0$, $114.7,110.1,106.9,101.3,55.6,47.3,29.7,29.1,22.4,14.0$.

IR $v_{\max } 2954(\mathrm{~m}), 2927(\mathrm{~m}), 2859(\mathrm{~m}), 1625(\mathrm{~m}), 1563$ (s), 1517 (s), 1498 (s), 1461 (s), 1387 (s), 1267 (s), 1233 (s), 1165 (s), 1112 (s), 850 (s), 779 (s), 739 (s).

HRMS calculated for $\mathrm{C}_{21} \mathrm{H}_{24} \mathrm{NO}_{3}{ }^{+}[\mathrm{M}+\mathrm{H}]^{+} 338.1751$; found 338.1753 .

\section{(2-Hydroxynaphthalen-1-yl)(1-pentyl-1H-indol-3-yl)methanone (6t)}

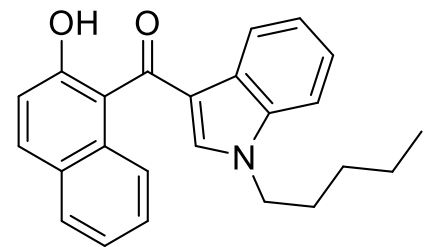

Following General Procedure E1, starting from commercially available 2hydroxy-1-naphthaldehyde and Pe-indoleBX 5b, a purification by column chromatography on silica gel (Pentane/EtOAc 6:1) afforded the title compound $\mathbf{6 t}$ (47.2 $\mathrm{mg}, 0.132 \mathrm{mmol}, 88 \%$ yield) as an off-white solid. $\mathbf{m p}$ $154-156{ }^{\circ}$ C. Rf 0.48 (Pentane/EtOAc 4:1).

1H NMR (400 MHz, $\left.\mathrm{CDCl}_{3}\right) \delta 9.87$ (bs, $\left.1 \mathrm{H}, \mathrm{OH}\right), 8.37-8.33\left(\mathrm{~m}, 1 \mathrm{H}, \underline{\mathrm{H}}_{\mathrm{Ar}}\right), 7.95\left(\mathrm{~d}, J=8.5 \mathrm{~Hz}, 1 \mathrm{H}, \underline{\mathrm{H}}_{\mathrm{Ar}}\right)$, $7.89\left(\mathrm{~d}, J=8.9 \mathrm{~Hz}, 1 \mathrm{H}, \underline{\mathrm{H}}_{\mathrm{Ar}}\right), 7.78\left(\mathrm{~d}, J=8.1 \mathrm{~Hz}, 1 \mathrm{H}, \underline{\mathrm{H}}_{\mathrm{Ar}}\right), 7.41-7.20\left(\mathrm{~m}, 7 \mathrm{H}, \mathrm{C} \underline{\mathrm{H}}-\mathrm{N}+\underline{\mathrm{H}}_{\mathrm{Ar}}\right), 4.02(\mathrm{t}, J=7.0$ $\left.\mathrm{Hz}, 2 \mathrm{H}, \mathrm{NCH}_{2}\right), 1.76(\mathrm{p}, J=7.1 \mathrm{~Hz}, 2 \mathrm{H}), 1.33-1.17(\mathrm{~m}, 4 \mathrm{H}), 0.86\left(\mathrm{t}, J=7.1 \mathrm{~Hz}, 3 \mathrm{H}, \mathrm{CH}_{3}\right)$.

${ }^{13}$ C NMR $\left(101 \mathrm{MHz}, \mathrm{CDCl}_{3}\right) \delta 192.3,157.4,138.5,137.0,133.7,132.7,128.9,128.4,127.0,126.3$ (2C), 123.8, 123.7, 123.3, 122.9, 119.2, 117.9, 117.5, 110.4, 47.4, 29.7, 29.0, 22.4, 14.1 .

IR $v_{\max } 3265$ (br), 2947 (m), 1588 (s), 1513 (s), 1462 (m), 1384 (s), 1177 (s), 1131 (s), 751 (s).

HRMS calculated for $\mathrm{C}_{24} \mathrm{H}_{24} \mathrm{NO}_{2}{ }^{+}[\mathrm{M}+\mathrm{H}]^{+} 358.1802$; found 358.1808.

\section{(1-(But-3-en-1-yl)-1H-indol-3-yl)(2-hydroxy-4-methoxyphenyl)methanone (6u)}<smiles>C=CCn1cc(C(=O)c2ccc(OC)cc2O)c2ccccc21</smiles>

Following General Procedure E1, but performing the reaction at $70{ }^{\circ} \mathrm{C}$, starting from commercially available 2-hydroxy-4-methoxybenzaldehyde and butenyl-indoleBX 5c, a purification by column chromatography on silica gel (Pentane/EtOAc 9:1) afford the title compound $6 \mathbf{u}(43.4 \mathrm{mg}, 0.135 \mathrm{mmol}$, $90 \%$ yield) as a yellow oil. Rf 0.56 (Pentane/EtOAc 4:1).

${ }^{1} \mathbf{H}$ NMR $\left(400 \mathrm{MHz}, \mathrm{CDCl}_{3}\right) \delta 12.83(\mathrm{bs}, 1 \mathrm{H}, \mathrm{O} \underline{\mathrm{H}}), 8.24-8.22\left(\mathrm{~m}, 1 \mathrm{H}, \underline{\mathrm{H}}_{\mathrm{Ar}}\right), 7.79\left(\mathrm{~d}, J=8.8 \mathrm{~Hz}, 1 \mathrm{H}, \underline{\mathrm{H}}_{\mathrm{Ar}}\right)$, $7.64(\mathrm{~s}, 1 \mathrm{H}, \mathrm{C} \underline{\mathrm{H}}-\mathrm{N}), 7.43-7.40\left(\mathrm{~m}, 1 \mathrm{H}, \underline{\mathrm{H}}_{\mathrm{Ar}}\right), 7.36-7.29\left(\mathrm{~m}, 2 \mathrm{H}, \underline{\mathrm{H}}_{\mathrm{Ar}}\right), 6.54\left(\mathrm{~d}, J=2.5 \mathrm{~Hz}, 1 \mathrm{H}, \underline{\mathrm{H}}_{\mathrm{Ar}}\right), 6.47$ $\left(\mathrm{dd}, J=8.9,2.5 \mathrm{~Hz}, 1 \mathrm{H}, \underline{\mathrm{H}}_{\mathrm{Ar}}\right), 5.80\left(\mathrm{ddt}, J=17.1,10.3,6.9 \mathrm{~Hz}, 1 \mathrm{H}, \underline{\mathrm{H}}_{\mathrm{Ar}}\right), 5.13-5.06\left(\mathrm{~m}, 2 \mathrm{H}, \underline{\mathrm{H}}_{\mathrm{Ar}}\right), 4.26(\mathrm{t}, J$ $\left.=7.0 \mathrm{~Hz}, 2 \mathrm{H}, \mathrm{NC}_{2}\right), 3.87\left(\mathrm{~s}, 3 \mathrm{H}, \mathrm{OCH}_{3}\right), 2.65\left(\mathrm{q}, J=7.0 \mathrm{~Hz}, 2 \mathrm{H}, \mathrm{NCH}_{2} \underline{\mathrm{C}}_{2}\right)$.

${ }^{13}$ C NMR $\left(101 \mathrm{MHz}, \mathrm{CDCl}_{3}\right) \delta 192.8,165.2,165.2,136.5,135.0,133.8,133.3,127.5,123.6,122.5,118.5$, $115.0,114.8,110.0,106.9,101.3,55.6,46.7,34.2$.

IR $v_{\max } 2927$ (w), 2852 (w), 1619 (s), 1582 (s), 1517 (s), 1461 (s), 1388 (s), 1365 (s), 1259 (s), 1231 (s), 1202 (s), 1160 (s), 1107 (s), 868 (s), 837 (s), 741 (s).

HRMS calculated for $\mathrm{C}_{20} \mathrm{H}_{20} \mathrm{NO}_{3}{ }^{+}[\mathrm{M}+\mathrm{H}]^{+} 322.1438$; found 322.1435 . 


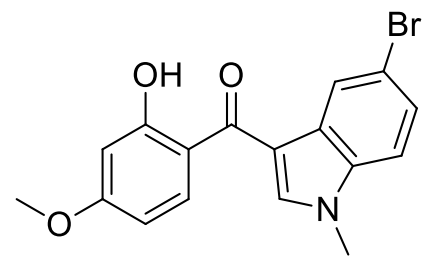

Following General Procedure E1, starting from commercially available 2hydroxy-4-methoxybenzaldehyde and Me-bromoindoleBX 5d, a purification by column chromatography on silica gel (Pentane/EtOAc 4:1) afforded the title compound $6 \mathbf{v}(46.5 \mathrm{mg}, 0.129 \mathrm{mmol}, 86 \%$ yield) as a yellow solid. $\mathbf{m p}$ 186- $188^{\circ} \mathrm{C}$. Rf 0.53 (Pentane/EtOAc 1:1).

${ }_{1}^{1} \mathbf{H}$ NMR $\left(400 \mathrm{MHz}, \mathrm{CDCl}_{3}\right) \delta 12.75(\mathrm{~s}, 1 \mathrm{H}, \mathrm{O} \underline{\mathrm{H}}), 8.37\left(\mathrm{~d}, J=1.8 \mathrm{~Hz}, 1 \mathrm{H}, \underline{\mathrm{H}}_{\mathrm{Ar}}\right), 7.73(\mathrm{~d}, J=8.8 \mathrm{~Hz}, 1 \mathrm{H}$, $\left.\underline{\mathrm{H}}_{\mathrm{Ar}}\right), 7.55(\mathrm{~s}, 1 \mathrm{H}, \mathrm{C} \underline{\mathrm{H}}-\mathrm{N}), 7.39\left(\mathrm{dd}, J=8.7,1.9 \mathrm{~Hz}, 1 \mathrm{H}, \underline{\mathrm{H}}_{\mathrm{Ar}}\right), 7.20\left(\mathrm{~d}, J=8.7 \mathrm{~Hz}, 1 \mathrm{H}, \underline{\mathrm{H}}_{\mathrm{Ar}}\right), 6.50(\mathrm{~d}, J=2.5$ $\left.\mathrm{Hz}, 1 \mathrm{H}, \underline{\mathrm{H}}_{\mathrm{Ar}}\right), 6.45\left(\mathrm{dd}, J=8.8,2.5 \mathrm{~Hz}, 1 \mathrm{H}, \underline{\mathrm{H}}_{\mathrm{Ar}}\right), 3.86\left(\mathrm{~s}, 3 \mathrm{H}, \mathrm{OC}_{3}\right), 3.85$ (s, $\left.3 \mathrm{H}, \mathrm{NC}_{3}\right)$.

${ }^{13} \mathrm{C}$ NMR $\left(101 \mathrm{MHz}, \mathrm{CDCl}_{3}\right) \delta 192.2,165.4,165.4,136.5,136.1,133.1,129.0,126.8,125.1,116.3,114.7$, $114.3,111.4,107.2,101.5,55.8,34.0$.

IR $v_{\max } 2920(\mathrm{~m}), 2852(\mathrm{~m}), 1628(\mathrm{~m}), 1574$ (s), 1504 (s), 1460 (s), 1338 (s), 1291 (s), 1233 (s), 1137 (s), 1113 (s), 1020 (s), 796 (s), 764 (s).

HRMS calculated for $\mathrm{C}_{17} \mathrm{H}_{15}{ }^{79} \mathrm{BrNO}_{3}{ }^{+}[\mathrm{M}+\mathrm{H}]^{+}$360.0230; found 360.0232.

\section{(2-Hydroxy-4-methoxyphenyl)(1H-indol-3-yl)methanone (6w)}<smiles>COc1ccc(C(=O)c2c[nH]c3ccccc23)c(O)c1</smiles>

Following General Procedure E1, but performing the reaction at $70{ }^{\circ} \mathrm{C}$, starting from commercially available 2-hydroxy-4-methoxybenzaldehyde and $\mathrm{NH}$-indoleBX 5e, a purification by column chromatography on silica gel (Pentane/EtOAc 4:1) afforded the title compound 6w (34.1 mg, $0.128 \mathrm{mmol}$, $85 \%$ yield) as a yellow solid. $\mathbf{m p ~} 175-177^{\circ} \mathrm{C}$. Rf 0.63 (Pentane/EtOAc 1:1). ${ }^{1} \mathbf{H}$ NMR $\left(400 \mathrm{MHz}, \mathrm{CDCl}_{3}\right) \delta 12.85(\mathrm{bs}, 1 \mathrm{H}, \mathrm{OH}), 8.73(\mathrm{~s}, 1 \mathrm{H}, \mathrm{N} \underline{\mathrm{H}}), 8.24-8.15\left(\mathrm{~m}, 1 \mathrm{H}, \underline{\mathrm{H}}_{\mathrm{Ar}}\right), 7.81(\mathrm{~d}, J=$ $\left.8.9 \mathrm{~Hz}, 1 \mathrm{H}, \underline{\mathrm{H}}_{\mathrm{Ar}}\right), 7.72\left(\mathrm{~d}, J=2.9 \mathrm{~Hz}, 1 \mathrm{H}, \underline{\mathrm{H}}_{\mathrm{Ar}}\right), 7.48-7.40\left(\mathrm{~m}, 1 \mathrm{H}, \underline{\mathrm{H}}_{\mathrm{Ar}}\right), 7.34-7.28\left(\mathrm{~m}, 2 \mathrm{H}, \underline{\mathrm{H}}_{\mathrm{Ar}}\right), 6.54(\mathrm{~d}, J$ $\left.=2.4 \mathrm{~Hz}, 1 \mathrm{H}, \underline{\mathrm{H}}_{\mathrm{Ar}}\right), 6.46\left(\mathrm{dd}, J=8.9,2.5 \mathrm{~Hz}, 1 \mathrm{H}, \underline{\mathrm{H}}_{\mathrm{Ar}}\right), 3.87\left(\mathrm{~s}, 3 \mathrm{H}, \mathrm{OC}_{3}\right)$.

${ }^{13} \mathrm{C}$ NMR $\left(101 \mathrm{MHz}, \mathrm{CDCl}_{3}\right) \delta 193.4,165.4(2 \mathrm{C}), 136.2,133.5,131.3,126.5,124.1,122.6,122.2,116.5$, 114.9, 111.6, 107.1, 101.4, 55.7.

IR $v_{\max } 3244$ (br), 1615 (m), 1567 (s), 1500 (s), 1425 (s), 1358 (s), 1195 (s), 1109 (s), 871 (s), 835 (s), 741 (s).

HRMS calculated for $\mathrm{C}_{16} \mathrm{H}_{14} \mathrm{NO}_{3}{ }^{+}[\mathrm{M}+\mathrm{H}]^{+}$268.0968; found 268.0980.

\section{(2-Hydroxy-4-methoxyphenyl)(1-methyl-1H-pyrrol-3-yl)methanone (6x)}<smiles>COc1ccc(C(=O)c2ccn(C)c2)c(O)c1</smiles>

Following General Procedure E1, starting from commercially available 2hydroxy-4-methoxybenzaldehyde and Me-pyrroleBX $\mathbf{5 f}$, a purification by column chromatography on silica gel (Pentane/EtOAc 4:1) afforded the title compound $\mathbf{6 x}$ (30.3 mg, $0.131 \mathrm{mmol}, 87 \%$ yield) as a pale yellow oil. Rf 0.31 (Pentane/EtOAc 4:1).

${ }^{1} \mathbf{H}$ NMR $\left(400 \mathrm{MHz}, \mathrm{CDCl}_{3}\right) \delta 12.99(\mathrm{~s}, 1 \mathrm{H}, \mathrm{OH}), 7.88\left(\mathrm{~d}, J=8.9 \mathrm{~Hz}, 1 \mathrm{H}, \underline{\mathrm{H}}_{\mathrm{Ar}}\right), 7.23(\mathrm{~s}, 1 \mathrm{H}, \mathrm{C} \underline{\mathrm{H}}-\mathrm{N}), 6.64$ $\left(\mathrm{t}, J=1.9 \mathrm{~Hz}, 2 \mathrm{H}, \underline{\mathrm{H}}_{\mathrm{Ar}}\right), 6.48\left(\mathrm{~d}, J=2.5 \mathrm{~Hz}, 1 \mathrm{H}, \underline{\mathrm{H}}_{\mathrm{Ar}}\right), 6.44\left(\mathrm{dd}, J=8.9,2.5 \mathrm{~Hz}, 1 \mathrm{H}, \underline{\mathrm{H}}_{\mathrm{Ar}}\right), 3.84(\mathrm{~s}, 3 \mathrm{H}$, $\left.\mathrm{OC}_{3}\right), 3.72\left(\mathrm{~s}, 3 \mathrm{H}, \mathrm{NCH}_{3}\right)$.

${ }^{13}$ C NMR $\left(101 \mathrm{MHz}, \mathrm{CDCl}_{3}\right) \delta 192.7,165.6,165.2,133.6,127.7,123.8,123.2,114.3,111.2,106.9,101.2$, $55.6,36.8$.

IR $v_{\max } 2927$ (w), 1608 (s), 1584 (s), 1524 (s), 1500 (s), 1443 (s), 1353 (s), 1236 (s), 1212 (s), 1149 (s), 1110 (s), 961 (s), 856 (s), 829 (s), 766 (s).

HRMS calculated for $\mathrm{C}_{13} \mathrm{H}_{14} \mathrm{NO}_{3}{ }^{+}[\mathrm{M}+\mathrm{H}]^{+} 232.0968$; found 232.0971 . 


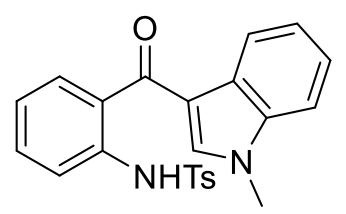

Following General Procedure E2, starting from $N$-(2-formylphenyl)4methylbenzenesulfonamide 7a and Me-indoleBX 5a, a purification by column chromatography on silica gel (Pentane/EtOAc 7:3) afforded the title compound 8a (53.3 mg, $0.132 \mathrm{mmol}, 88 \%$ yield) as a white solid. $\mathbf{m p ~} 75-77{ }^{\circ} \mathrm{C}$. Rf 0.47 (Pentane/EtOAc 1:1).

1 H NMR $\left(400 \mathrm{MHz}, \mathrm{CDCl}_{3}\right) \delta 9.61(\mathrm{~s}, 1 \mathrm{H}, \mathrm{NHTs}), 8.19\left(\mathrm{~d}, J=7.3 \mathrm{~Hz}, 1 \mathrm{H}, \underline{\mathrm{H}}_{\mathrm{Ar}}\right), 7.72(\mathrm{~d}, J=8.2,0.8 \mathrm{~Hz}$, $\left.2 \mathrm{H}, \underline{\mathrm{H}}_{\mathrm{Ar}}\right), 7.53\left(\mathrm{dd}, J=7.7,1.5 \mathrm{~Hz}, 1 \mathrm{H}, \underline{\mathrm{H}}_{\mathrm{Ar}}\right), 7.51-7.43\left(\mathrm{~m}, 3 \mathrm{H}, \underline{\mathrm{H}}_{\mathrm{tolyl}}+\underline{\mathrm{H}}_{\mathrm{Ar}}\right), 7.38-7.31\left(\mathrm{~m}, 3 \mathrm{H}, \underline{\mathrm{H}}_{\mathrm{Ar}}\right), 7.15$ $\left(\mathrm{td}, J=7.6,1.1 \mathrm{~Hz}, 1 \mathrm{H}, \underline{\mathrm{H}}_{\mathrm{Ar}}\right), 7.09(\mathrm{~s}, 1 \mathrm{H}, \mathrm{C} \underline{\mathrm{H}}-\mathrm{N}), 6.79\left(\mathrm{~d}, J=8.0 \mathrm{~Hz}, 2 \mathrm{H}, \underline{\mathrm{H}}_{\text {tolyl }}\right), 3.81\left(\mathrm{~s}, 3 \mathrm{H}, \mathrm{NCH}_{3}\right), 2.05$ (s, $\left.3 \mathrm{H}, \mathrm{CH}_{3}\right)$.

${ }^{13}$ C NMR $\left(101 \mathrm{MHz}, \mathrm{CDCl}_{3}\right) \delta 190.8,143.1,138.5,137.4,137.2,136.2,132.1,130.6,130.3,129.2,127.4$, $127.0,124.1,124.0,123.9,123.1,122.7,115.8,109.8,33.7,21.4$.

IR $v_{\max } 3216(\mathrm{w}), 2920(\mathrm{w}), 1595(\mathrm{~m}), 1521(\mathrm{~s}), 1487(\mathrm{~m}), 1466(\mathrm{~s}), 1396(\mathrm{~m}), 1365(\mathrm{~s}), 1334(\mathrm{~s}), 1233(\mathrm{~s})$, 1159 (s), 1126 (m), 1092 (m), 1073 (m), 925 (m), 869 (s), $814(\mathrm{~m}), 747$ (s).

HRMS calculated for $\mathrm{C}_{23} \mathrm{H}_{20} \mathrm{~N}_{2} \mathrm{NaO}_{3} \mathrm{~S}^{+}[\mathrm{M}+\mathrm{Na}]^{+} 427.1087$; found 427.1095.

\section{$N$-(2-(1-Methyl-1H-indole-3-carbonyl)phenyl)-4-nitrobenzenesulfonamide (8b)}

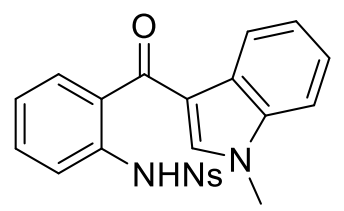

Following General Procedure E2, starting from $N$-(2-formylphenyl)4nitrobenzenesulfonamide $\mathbf{7 b}$ and Me-indoleBX 5a, a purification by column chromatography on silica gel (Pentane/EtOAc/DCM 6:3:1) afforded the title compound $8 \mathbf{b}$ (52.0 mg, $0.119 \mathrm{mmol}, 80 \%$ yield) or (280 mg, $0.643 \mathrm{mmol}, 80 \%$ yield) as a yellow solid. $\mathbf{m p} 212-214{ }^{\circ} \mathrm{C}$. Rf 0.67 (Pentane/EtOAc 1:1).

$\mathbf{1}_{\mathbf{H}} \mathbf{N M R}\left(400 \mathrm{MHz}, \mathrm{CDCl}_{3}\right) \delta 9.46$ (s, $\left.1 \mathrm{H}, \mathrm{NHNs}\right), 8.17\left(\mathrm{~d}, J=7.1 \mathrm{~Hz}, 1 \mathrm{H}, \underline{\mathrm{H}}_{\mathrm{Ar}}\right), 7.77(\mathrm{~d}, J=8.1 \mathrm{~Hz}, 1 \mathrm{H}$, $\left.\underline{\mathrm{H}}_{\mathrm{Ar}}\right), 7.68\left(\mathrm{~d}, J=8.9 \mathrm{~Hz}, 2 \mathrm{H}, \underline{\mathrm{H}}_{\mathrm{nosyl}}\right), 7.60\left(\mathrm{~d}, J=8.9 \mathrm{~Hz}, 2 \mathrm{H}, \underline{\mathrm{H}}_{\mathrm{nosyl}}\right), 7.58-7.51\left(\mathrm{~m}, 2 \mathrm{H}, \underline{\mathrm{H}}_{\mathrm{Ar}}\right), 7.43-7.34(\mathrm{~m}$, $\left.2 \mathrm{H}, \underline{\mathrm{H}}_{\mathrm{Ar}}\right), 7.33-7.27$ (m, 2H, $\left.\underline{\mathrm{H}}_{\mathrm{Ar}}\right), 6.97$ (s, $\left.1 \mathrm{H}, \mathrm{C} \underline{\mathrm{H}}-\mathrm{N}\right), 3.73$ (s, 3H, NC$\left.\underline{\mathrm{H}}_{3}\right)$.

13C NMR (101 MHz, $\left.\mathrm{CDCl}_{3}\right) \delta 190.0,149.2,144.7,138.3,137.2,135.5,132.2,132.1,129.8,128.7,126.8$, 126.6, 125.8, 124.6, 123.7, 123.5, 122.6, 115.6, 110.2, 33.6.

IR $v_{\max } 3105(\mathrm{w}), 2933(\mathrm{w}), 1737(\mathrm{w}), 1608$ (m), $1595(\mathrm{~m}), 1527$ (s), 1466 (m), 1404 (m), 1367 (s), 1349 (s), $1232(\mathrm{~m}), 1164(\mathrm{~s}), 874(\mathrm{~m}), 856(\mathrm{~m}), 763(\mathrm{~s}), 757(\mathrm{~m}), 739(\mathrm{~s}), 712(\mathrm{~m})$.

HRMS calculated for $\mathrm{C}_{22} \mathrm{H}_{17} \mathrm{~N}_{3} \mathrm{NaO}_{5} \mathrm{~S}^{+}[\mathrm{M}+\mathrm{Na}]^{+} 458.0781$; found 458.0780.

\section{$N$-(2-(1-Methyl-1H-indole-3-carbonyl)phenyl)methanesulfonamide (8c)}

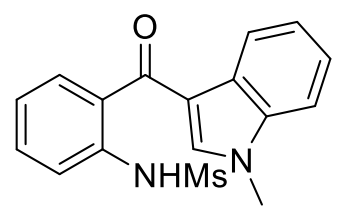

Following General Procedure E2, starting from $N$-(2-formylphenyl)4methylsulfonamide $\mathbf{7 c}$ and Me-indoleBX 5a, a purification by column chromatography on silica gel (Pentane/EtOAc 7:3) afforded the title compound 8c (42.6 mg, $0.130 \mathrm{mmol}, 86 \%$ yield) as a white solid. $\mathbf{m p} 132-134{ }^{\circ} \mathrm{C}$. $\mathbf{R f} 0.41$ (Pentane/EtOAc 1:1).

$1 \mathbf{H}$ NMR $\left(400 \mathrm{MHz}, \mathrm{CDCl}_{3}\right) \delta 9.63(\mathrm{~s}, 1 \mathrm{H}, \mathrm{NHMs}), 8.37-8.31\left(\mathrm{~m}, 1 \mathrm{H}, \underline{\mathrm{H}}_{\mathrm{Ar}}\right), 7.79-7.75\left(\mathrm{~m}, 2 \mathrm{H}, \underline{\mathrm{H}}_{\mathrm{Ar}}\right), 7.55-$ $7.51\left(\mathrm{~m}, 2 \mathrm{H}, \mathrm{C} \underline{\mathrm{H}}-\mathrm{N}+\underline{\mathrm{H}}_{\mathrm{Ar}}\right), 7.41-7.34\left(\mathrm{~m}, 3 \mathrm{H}, \underline{\mathrm{H}}_{\mathrm{Ar}}\right), 7.22\left(\mathrm{t}, J=7.6 \mathrm{~Hz}, 1 \mathrm{H}, \underline{\mathrm{H}}_{\mathrm{Ar}}\right), 3.87\left(\mathrm{~s}, 3 \mathrm{H}, \mathrm{NC}_{3}\right), 2.97$ (s, $\left.3 \mathrm{H}, \mathrm{CH}_{3}\right)$.

${ }^{13}$ C NMR $\left(101 \mathrm{MHz}, \mathrm{CDCl}_{3}\right) \delta 191.3,138.6,138.2,137.8,132.8,131.6,128.2,127.2,124.3,123.5,123.4$, $122.7,121.0,116.0,110.2,40.1,34.0$.

IR $v_{\max } 3216$ (br), 2933 (w), 1608 (m), 1521 (s), 1488 (s), 1464 (s), 1363 (s), 1327 (s), 1233 (s), 1149 (s), 1127 (s), 1104 (m), 1074 (m), 969 (s), 924 (m), 868 (s), 744 (s).

HRMS calculated for $\mathrm{C}_{17} \mathrm{H}_{16} \mathrm{~N}_{2} \mathrm{NaO}_{3} \mathrm{~S}^{+}[\mathrm{M}+\mathrm{Na}]^{+}$351.0774; found 351.0770. 
<smiles></smiles>

Following General Procedure E2, starting from $N$-(4-chloro-2-formylphenyl)4methylbenzenesulfonamide 7d and Me-indoleBX 5a, a purification by column chromatography on silica gel (Pentane/EtOAc 7:3) afforded the title compound $8 d$ (59.9 mg, $0.136 \mathrm{mmol}, 91 \%$ yield) as a white solid. $\mathbf{m p} 193-195{ }^{\circ} \mathrm{C}$. $\mathbf{R f} 0.68$ (Pentane/EtOAc 1:1).

${ }_{1}^{1}$ H NMR $\left(400 \mathrm{MHz}, \mathrm{CDCl}_{3}\right) \delta 9.36(\mathrm{~s}, 1 \mathrm{H}, \mathrm{NHTs}), 8.21-8.18\left(\mathrm{~m}, 1 \mathrm{H}, \underline{\mathrm{H}}_{\mathrm{Ar}}\right), 7.68\left(\mathrm{~d}, J=8.7 \mathrm{~Hz}, 1 \mathrm{H}, \underline{\mathrm{H}}_{\mathrm{Ar}}\right)$, 7.50-7.46 (m, 3H, $\left.\underline{\mathrm{H}}_{\mathrm{tolyl}}+\underline{\mathrm{H}}_{\mathrm{Ar}}\right), 7.43\left(\mathrm{dd}, J=8.7,2.4 \mathrm{~Hz}, 1 \mathrm{H}, \underline{\mathrm{H}}_{\mathrm{Ar}}\right), 7.40-7.33\left(\mathrm{~m}, 3 \mathrm{H}, \underline{\mathrm{H}}_{\mathrm{Ar}}\right), 7.08(\mathrm{~s}, 1 \mathrm{H}$, $\mathrm{C} \underline{\mathrm{H}}-\mathrm{N}), 6.79\left(\mathrm{~d}, J=8.1 \mathrm{~Hz}, 2 \mathrm{H}, \underline{\mathrm{H}}_{\text {tolyl }}\right), 3.83\left(\mathrm{~s}, 3 \mathrm{H}, \mathrm{NC}_{3}\right), 2.05$ (s, 3H, $\left.\underline{\mathrm{C}}_{3}\right)$.

${ }^{13} \mathrm{C}$ NMR $\left(101 \mathrm{MHz}, \mathrm{CDCl}_{3}\right) \delta 189.0,143.3,138.4,137.5,136.0,135.6,131.9,131.8,130.0,129.8,129.3$, $127.4,126.9,125.8,124.3,123.5,122.7,115.5,109.9,33.9,21.4$.

IR $v_{\max } 3228(\mathrm{~m}), 1608(\mathrm{~m}), 1594(\mathrm{~m}), 1523(\mathrm{~s}), 1469(\mathrm{~m}), 1396(\mathrm{~m}), 1385(\mathrm{~m}), 1362(\mathrm{~s}), 1328(\mathrm{~s}), 1233$ (m), $1160(\mathrm{~s}), 1129(\mathrm{~m}), 1090(\mathrm{~s}), 878(\mathrm{~s}), 814(\mathrm{~s}), 750(\mathrm{~s}), 715(\mathrm{~s})$.

HRMS calculated for $\mathrm{C}_{23} \mathrm{H}_{19} \mathrm{ClN}_{2} \mathrm{NaO}_{3} \mathrm{~S}^{+}[\mathrm{M}+\mathrm{Na}]^{+} 461.0697$; found 461.0701 .

\section{4-Methyl-N-(2-(1-methyl-1H-indole-3-carbonyl)thiophen-3-yl)benzenesulfonamide (8e)}<smiles>Cn1cc(C(=O)c2sccc2N)c2ccccc21</smiles>

Following General Procedure E2, starting from $N$-(2-formylthiophen-3-yl)-4methylbenzenesulfonamide 7e and Me-indoleBX 5a, a purification by column chromatography on silica gel (Pentane/EtOAc 3:2) afforded the title compound 8e (55.5 mg, $0.135 \mathrm{mmol}, 90 \%$ yield) as a yellow solid. $\mathbf{m p} 158-160{ }^{\circ} \mathrm{C}$. Rf 0.50 (Pentane/EtOAc 1:1).

${ }^{1} \mathbf{H}$ NMR $\left(400 \mathrm{MHz}, \mathrm{CDCl}_{3}\right) \delta 11.32(\mathrm{~s}, 1 \mathrm{H}, \mathrm{NHTs}), 8.36\left(\mathrm{~d}, J=5.9 \mathrm{~Hz}, 1 \mathrm{H}, \underline{\mathrm{H}}_{\mathrm{Ar}}\right), 7.87(\mathrm{~s}, 1 \mathrm{H}, \mathrm{C} \underline{\mathrm{H}}-\mathrm{N}), 7.78$ $\left(\mathrm{d}, J=7.8 \mathrm{~Hz}, 2 \mathrm{H}, \underline{\mathrm{H}}_{\mathrm{toly}}\right), 7.47\left(\mathrm{~d}, J=5.4 \mathrm{~Hz}, 1 \mathrm{H}, \underline{\mathrm{H}}_{\mathrm{Ar}}\right), 7.38\left(\mathrm{~d}, J=5.4 \mathrm{~Hz}, 1 \mathrm{H}, \underline{\mathrm{H}}_{\mathrm{Ar}}\right), 7.34-7.29(\mathrm{~m}, 3 \mathrm{H}$, $\left.\underline{\mathrm{H}}_{\mathrm{Ar}}\right), 7.20\left(\mathrm{~d}, J=7.8 \mathrm{~Hz}, 2 \mathrm{H}, \underline{\mathrm{H}}_{\text {tolyl }}\right), 3.84$ (s, 3H, NC$\left.\underline{\mathrm{H}}_{3}\right), 2.32$ (s, 3H, $\left.\underline{\mathrm{C}}_{3}\right)$.

${ }^{13} \mathrm{C}$ NMR $\left(101 \mathrm{MHz}, \mathrm{CDCl}_{3}\right) \delta 182.9,143.9,137.3,136.9,135.0,130.8,129.8,127.4,127.2,124.0,122.9$, $122.7,121.3,119.2,115.8,109.9,33.8,21.6$. One aromatic $\mathrm{C}$ is not resolved.

IR $v_{\max } 3117$ (w), 3087 (w), 1554 (s), 1515 (s), 1463 (s), 1399 (s), 1370 (s), 1344 (s), 1227 (s), 1152 (s), 1087 (s), 862 (s), 815 (s), 748 (s).

HRMS calculated for $\mathrm{C}_{21} \mathrm{H}_{19} \mathrm{~N}_{2} \mathrm{O}_{3} \mathrm{~S}_{2}{ }^{+}[\mathrm{M}+\mathrm{H}]^{+} 411.0832$; found 411.0832 .

\section{$N$-(2-(1-(But-3-en-1-yl)-1H-indole-3-carbonyl)phenyl)-4-methylbenzenesulfonamide (8f)}

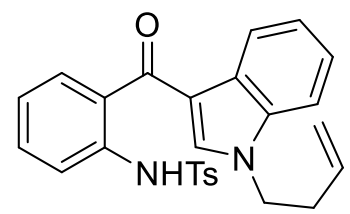

Following General Procedure E2, starting from $N$-(2-formylphenyl)4methylbenzenesulfonamide 7a and butenyl-indoleBX 5d, a purification by column chromatography on silica gel (Pentane/EtOAc 7:3) afforded the title compound 8d (52.7 mg, $0.119 \mathrm{mmol}, 79 \%$ yield) as a pale yellow solid. $\mathbf{m p} 50-52{ }^{\circ} \mathrm{C}$. $\mathbf{R f} 0.80$ (Pentane/EtOAc 1:1).

1 H NMR $\left(400 \mathrm{MHz}, \mathrm{CDCl}_{3}\right) \delta 9.74(\mathrm{~s}, 1 \mathrm{H}, \mathrm{NHTs}), 8.18\left(\mathrm{~d}, J=7.0 \mathrm{~Hz}, 1 \mathrm{H}, \underline{\mathrm{H}}_{\mathrm{Ar}}\right), 7.73(\mathrm{~d}, J=8.2 \mathrm{~Hz}, 1 \mathrm{H}$, $\left.\underline{\mathrm{H}}_{\mathrm{Ar}}\right), 7.57-7.53\left(\mathrm{~m}, 3 \mathrm{H}, \underline{\mathrm{H}}_{\mathrm{tolyl}}+\underline{\mathrm{H}}_{\mathrm{Ar}}\right), 7.46\left(\mathrm{td}, J=8.1,1.5 \mathrm{~Hz}, 1 \mathrm{H}, \underline{\mathrm{H}}_{\mathrm{Ar}}\right), 7.41-7.30\left(\mathrm{~m}, 3 \mathrm{H}, \underline{\mathrm{H}}_{\mathrm{Ar}}\right), 7.21(\mathrm{~s}, 1 \mathrm{H}$, C $\underline{\mathrm{H}}-\mathrm{N}), 7.14\left(\mathrm{td}, J=7.6,1.1 \mathrm{~Hz}, 1 \mathrm{H}, \underline{\mathrm{H}}_{\mathrm{Ar}}\right), 6.87\left(\mathrm{~d}, J=8.0 \mathrm{~Hz}, 2 \mathrm{H}, \underline{\mathrm{H}}_{\text {tolyl }}\right), 5.76$ (ddt, $J=17.1,10.3,6.9 \mathrm{~Hz}$, $\left.1 \mathrm{H}, \mathrm{C} \underline{\mathrm{H}}=\mathrm{CH}_{2}\right), 5.15-5.05\left(\mathrm{~m}, 2 \mathrm{H}, \underline{\mathrm{C}} \underline{\mathrm{H}}=\mathrm{CH}_{2}\right), 4.19\left(\mathrm{t}, J=7.1 \mathrm{~Hz}, 2 \mathrm{H}, \mathrm{NC}_{2}\right), 2.60(\mathrm{q}, J=7.0 \mathrm{~Hz}, 2 \mathrm{H}$, $\left.\mathrm{NCH}_{2} \underline{\mathrm{C}}_{2}\right), 2.08$ (s, $\left.3 \mathrm{H}, \mathrm{C}_{3}\right)$.

${ }^{13}$ C NMR $\left(101 \mathrm{MHz}, \mathrm{CDCl}_{3}\right) \delta 191.1,143.3,137.6,137.6,136.7,136.5,133.7,132.3,130.9,129.8$, $129.4,127.5,127.3,124.1,123.9,123.3,123.2,123.0,118.8,116.0,110.1,46.8,34.3,21.5$.

IR $v_{\max } 3672(\mathrm{w}), 3222(\mathrm{br}), 2976(\mathrm{~m}), 2914(\mathrm{~m}), 1607$ (m), 1599 (m), 1520 (s), 1486 (m), 1466 (m), 1394 (s), 1377 (s), $1335(\mathrm{~m}), 1158(\mathrm{~s}), 1090(\mathrm{~s}), 1048(\mathrm{~m}), 922(\mathrm{~m}), 868(\mathrm{~s}), 735(\mathrm{~s})$.

HRMS calculated for $\mathrm{C}_{26} \mathrm{H}_{25} \mathrm{~N}_{2} \mathrm{O}_{3} \mathrm{~S}^{+}[\mathrm{M}+\mathrm{H}]^{+} 445.1580$; found 445.1576 . 


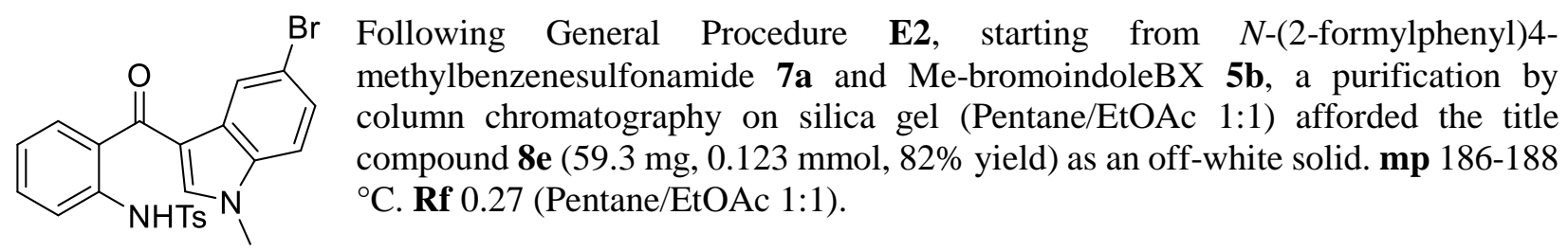

${ }_{1}^{1} \mathbf{H}$ NMR $\left(400 \mathrm{MHz}, \mathrm{CDCl}_{3}\right) \delta 9.55(\mathrm{~s}, 1 \mathrm{H}, \mathrm{N} \underline{\mathrm{HT}}), 8.30\left(\mathrm{~d}, J=1.6 \mathrm{~Hz}, 1 \mathrm{H}, \underline{\mathrm{H}}_{\mathrm{Ar}}\right), 7.72(\mathrm{dd}, J=8.2,0.8$ $\left.\mathrm{Hz}, 1 \mathrm{H}, \underline{\mathrm{H}}_{\mathrm{Ar}}\right), 7.53-7.43\left(\mathrm{~m}, 5 \mathrm{H}, \underline{\mathrm{H}}_{\mathrm{tolyl}}+\underline{\mathrm{H}}_{\mathrm{Ar}}\right), 7.22\left(\mathrm{~d}, J=8.5 \mathrm{~Hz}, 1 \mathrm{H}, \underline{\mathrm{H}}_{\mathrm{Ar}}\right), 7.16(\mathrm{td}, J=7.6,1.1 \mathrm{~Hz}, 1 \mathrm{H}$, $\left.\underline{\mathrm{H}}_{\mathrm{Ar}}\right), 7.12$ (s, $\left.1 \mathrm{H}, \mathrm{C} \underline{\mathrm{H}}-\mathrm{N}\right), 6.85\left(\mathrm{~d}, J=8.0 \mathrm{~Hz}, 2 \mathrm{H}, \underline{\mathrm{H}}_{\text {tolyl }}\right), 3.80\left(\mathrm{~s}, 3 \mathrm{H}, \underline{\mathrm{NC}}_{3}\right), 2.07$ (s, 3H, $\left.\underline{\mathrm{C}}_{3}\right)$.

${ }^{13}$ C NMR $\left(101 \mathrm{MHz}, \mathrm{CDCl}_{3}\right) \delta 190.6,143.2,138.8,137.3,136.3,136.1,132.4,130.5,129.8,129.3,128.5$, $127.4,127.1,125.3,124.1,124.0,116.9,115.3,111.3,33.9,21.4$.

IR $v_{\max } 3222(\mathrm{br}), 2933(\mathrm{~m}), 1607$ (m), 1596 (s), 1525 (s), 1486 (m), 1460 (s), 1450 (m), 1390 (m), 1366 (s), 1332 (s), 1232 (s), 1163 (s), $1090(\mathrm{~m}), 877$ (m), 803 (s), 763 (s), 719 (s).

HRMS calculated for $\mathrm{C}_{23} \mathrm{H}_{20}{ }^{79} \mathrm{BrN}_{2} \mathrm{O}_{3} \mathrm{~S}^{+}[\mathrm{M}+\mathrm{H}]^{+} 483.0373$; found 483.0372 . 


\section{Product modifications}

\section{2-(1-Methyl-1H-indole-3-carbonyl)phenyl trifluoromethanesulfonate (9)}<smiles>Cn1cc(C(=O)c2ccccc2)c2ccccc21</smiles>

\author{
$\underset{\mathrm{DCM}, 1 \mathrm{~h}, \mathrm{rt}}{\stackrel{\mathrm{Tf}_{2} \mathrm{O}, \text { pyridine }}{\longrightarrow}}$
}

$6 a$<smiles>COc1ccccc1C(=O)c1cn(C)c2ccccc12</smiles>

9

6a (126 mg, $0.500 \mathrm{mmol}, 1.00$ equiv.) and pyridine ( $80 \mu \mathrm{L}, 1.0 \mathrm{mmol}, 2.0$ equiv.) were solubilized in DCM $(2.5 \mathrm{~mL})$. Triflic anhydride $(100 \mu \mathrm{L}, 0.600 \mathrm{mmol}, 1.20$ equiv. $)$ was added at $0{ }^{\circ} \mathrm{C}$. The mixture was stirred $1 \mathrm{~h}$ at $\mathrm{rt}$ and then quenched with $\mathrm{H}_{2} \mathrm{O}$. The layers were separated and the aqueous layer was extracted three times with DCM $(10 \mathrm{~mL})$. The combined organic layers were washed with brine, dried over $\mathrm{MgSO}_{4}$ and concentrated under reduced pressure. A purification by column chromatography on silica gel (Pentane/EtOAc 7:3) afford the title compound 9 (192 mg, $0.500 \mathrm{mmol}, 100 \%$ yield) as a white solid. mp $148-150{ }^{\circ} \mathrm{C}$. Rf 0.66 (Pentane/EtOAc 1:1).

${ }^{1} \mathbf{H}$ NMR $\left(400 \mathrm{MHz}, \mathrm{CDCl}_{3}\right) \delta 8.40-8.33\left(\mathrm{~m}, 1 \mathrm{H}, \underline{\mathrm{H}}_{\mathrm{Ar}}\right), 7.64\left(\mathrm{dd}, J=7.5,1.8 \mathrm{~Hz}, 1 \mathrm{H}, \underline{\mathrm{H}}_{\mathrm{Ar}}\right), 7.61-7.55(\mathrm{~m}$, $\left.1 \mathrm{H}, \underline{\mathrm{H}}_{\mathrm{Ar}}\right), 7.50-7.45\left(\mathrm{~m}, 1 \mathrm{H}, \underline{\mathrm{H}}_{\mathrm{Ar}}\right), 7.42\left(\mathrm{~d}, J=8.3 \mathrm{~Hz}, 1 \mathrm{H}, \underline{\mathrm{H}}_{\mathrm{Ar}}\right), 7.38-7.33\left(\mathrm{~m}, 4 \mathrm{H}, \mathrm{C} \underline{\mathrm{H}}-\mathrm{N}+\underline{\mathrm{H}}_{\mathrm{Ar}}\right), 3.83(\mathrm{~s}$, $\left.3 \mathrm{H}, \mathrm{NC}_{3}\right) .{ }^{19} \mathbf{F}$ NMR $\left(376 \mathrm{MHz}, \mathrm{CDCl}_{3}\right) \delta-73.6 .{ }^{13} \mathbf{C} \mathbf{N M R}\left(101 \mathrm{MHz}, \mathrm{CDCl}_{3}\right) \delta 185.7,146.5,138.7$, 137.9, 135.0, 131.6, 130.6, 128.2, 126.7, 124.1, 123.3, 122.8, 122.5, 118.6 (q, $J=320 \mathrm{~Hz}$ ), 116.4, 109.9, 33.8. IR $v_{\max } 1620(\mathrm{~s}), 1525(\mathrm{~m}), 1466(\mathrm{~m}), 1422(\mathrm{~s}), 1369(\mathrm{~m}), 1237(\mathrm{~m}), 1215(\mathrm{~s}), 1206(\mathrm{~s}), 1144(\mathrm{~s}), 1096$ (m), $1073(\mathrm{~m}), 907$ (s), 881 (m), 853 (s), 772 (s), 759 (s), $732(\mathrm{~m})$. HRMS calculated for $\mathrm{C}_{17} \mathrm{H}_{13} \mathrm{~F}_{3} \mathrm{NO}_{4} \mathrm{~S}^{+}$ $[\mathrm{M}+\mathrm{H}]^{+}$384.0512; found 384.0507.

\section{[1,1'-Biphenyl]-2-yl(1-methyl-1H-indol-3-yl)methanone (10)}<smiles>COc1ccccc1C(=O)c1cn(C)c2ccccc12</smiles>

9

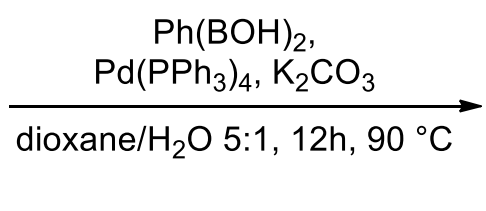

$\mathrm{Ph}(\mathrm{BOH})_{2}$,

dioxane $/ \mathrm{H}_{2} \mathrm{O} 5: 1,12 \mathrm{~h}, 90{ }^{\circ} \mathrm{C}$

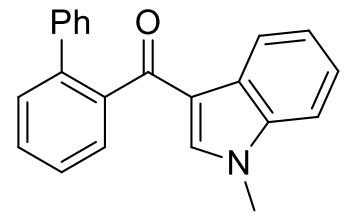

10

9 (90.0 mg, $0.235 \mathrm{mmol}, 1.00$ equiv.), $\mathrm{PhB}(\mathrm{OH})_{2}\left(29.0 \mathrm{mg}, 0.238 \mathrm{mmol}, 1.00\right.$ equiv.), $\mathrm{K}_{2} \mathrm{CO}_{3}(64.9 \mathrm{mg}$, $0.470 \mathrm{mmol}, 2.00$ equiv. $)$ and $\mathrm{Pd}\left(\mathrm{PPh}_{3}\right)_{4}(8.1 \mathrm{mg}, 7.0 \mu \mathrm{mol}, 3 \mathrm{~mol} \%)$ were solubilized in dioxane $(1.0 \mathrm{~mL}) /$ water $(0.2 \mathrm{~mL})$ previously degazed by bubbling $\mathrm{N}_{2}$. The mixture was stirred $12 \mathrm{~h}$ at $90{ }^{\circ} \mathrm{C}$ and then quenched with $\mathrm{H}_{2} \mathrm{O}$. The layers were separated and the aqueous layer was extracted three times with EtOAc $(10 \mathrm{~mL})$. The combined organic layers were washed with brine, dried over $\mathrm{MgSO}_{4}$ and concentrated under reduced pressure. A purification by column chromatography on silica gel (Pentane/EtOAc 4:1) afforded the title compound 10 (65.2 mg, $0.209 \mathrm{mmol}, 89 \%$ yield) as a white solid. mp 174-176 ${ }^{\circ} \mathrm{C}$. Rf 0.80 (Pentane/EtOAc $1: 1)$.

${ }^{1} \mathbf{H}$ NMR $\left(400 \mathrm{MHz}, \mathrm{CDCl}_{3}\right) \delta 8.23\left(\mathrm{~d}, J=8.2 \mathrm{~Hz}, 1 \mathrm{H}, \underline{\mathrm{H}}_{\mathrm{Ar}}\right), 7.57-7.48\left(\mathrm{~m}, 3 \mathrm{H}, \underline{\mathrm{H}}_{\mathrm{Ar}}\right), 7.45-7.37(\mathrm{~m}, 3 \mathrm{H}$, $\left.\underline{\mathrm{H}}_{\mathrm{Ar}}\right), 7.33-7.13\left(\mathrm{~m}, 7 \mathrm{H}, \mathrm{C} \underline{\mathrm{H}}-\mathrm{N}+\underline{\mathrm{H}}_{\mathrm{Ar}}\right), 3.70\left(\mathrm{~s}, 3 \mathrm{H}, \mathrm{NC}_{3}\right) .{ }^{13} \mathbf{C} \mathbf{N M R}\left(101 \mathrm{MHz}, \mathrm{CDCl}_{3}\right) \delta 192.5,141.2$, 140.7, 140.3, 138.6, 137.6, 130.4, 129.7, 128.9, 128.5, 128.4, 127.3, 127.0, 126.7, 123.5, 122.7 (2C), 117.2, 109.6, 33.5. IR $v_{\max } 3105$ (w), 3050 (w), 3031 (w), 1620 (s), 1524 (s), 1463 (m), 1369 (s), 1228 (s), 1124 (m), $1073(\mathrm{~m}), 887(\mathrm{~m}), 870(\mathrm{~m}), 743$ (s). HRMS calculated for $\mathrm{C}_{22} \mathrm{H}_{18} \mathrm{NO}^{+}[\mathrm{M}+\mathrm{H}]^{+}$312.1383; found 312.1386. 
<smiles>CCOc1ccccc1C(=O)c1cn(C)c2ccccc12</smiles>

9

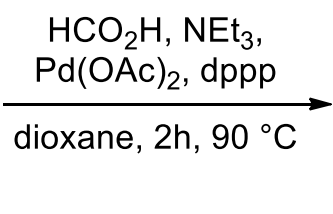

Pd(OAc)

dioxane, $2 \mathrm{~h}, 90^{\circ} \mathrm{C}$<smiles>Cn1cc(C(=O)c2ccccc2)c2ccccc21</smiles>

11

9 (38.3 mg, $0.100 \mathrm{mmol}, 1.00$ equiv.), $\mathrm{Pd}(\mathrm{OAc})_{2}(2.2 \mathrm{mg}, 10 \mu \mathrm{mol}, 0.10$ equiv) and dppp (12.4 mg, 30.0 $\mu$ mol, 0.300 equiv) were solubilized in dioxane $(1.0 \mathrm{~mL})$ previously degazed by bubbling $\mathrm{N}_{2}$. Formic acid ( $15 \mu \mathrm{L}, 0.40 \mathrm{mmol}, 4.0$ equiv.) and triethylamine ( $55 \mu \mathrm{L}, 0.40 \mathrm{mmol}, 4.0$ equiv.) were added to the solution. The mixture was stirred $2 \mathrm{~h}$ at $90^{\circ} \mathrm{C}$ and then quenched with $\mathrm{H}_{2} \mathrm{O}$. The layers were separated and the aqueous layer was extracted three times with EtOAc $(10 \mathrm{~mL})$. The combined organic layers were washed with brine, dried over $\mathrm{MgSO}_{4}$ and concentrated under reduced pressure. A purification by column chromatography on silica gel (Pentane/EtOAc 4:1) afforded the title compound 11 (22.9 mg, $97.0 \mu \mathrm{mol}, 97 \%$ yield) as a white solid. ${ }^{1} \mathbf{H}$ NMR $\left(400 \mathrm{MHz}, \mathrm{CDCl}_{3}\right) \delta 8.48-8.41\left(\mathrm{~m}, 1 \mathrm{H}, \underline{\mathrm{H}}_{\mathrm{Ar}}\right), 7.81\left(\mathrm{~d}, J=7.0 \mathrm{~Hz}, 2 \mathrm{H}, \underline{\mathrm{H}}_{\mathrm{Ar}}\right), 7.59-7.44(\mathrm{~m}$, $\left.4 \mathrm{H}, \underline{\mathrm{H}}_{\mathrm{Ar}}\right), 7.39-7.32\left(\mathrm{~m}, 3 \mathrm{H}, \underline{\mathrm{H}}_{\mathrm{Ar}}\right), 3.83\left(\mathrm{~s}, 3 \mathrm{H}, \mathrm{NC}_{3}\right) .{ }^{13} \mathbf{C} \mathbf{N M R}\left(101 \mathrm{MHz}, \mathrm{CDCl}_{3}\right) \delta$ 191.0, 141.0, 138.0, 137.6, 131.2, 128.8, 128.4, 127.3, 123.7, 122.8, 122.8, 115.7, 109.7, 33.7. Spectra data matched with the values reported in literature. ${ }^{17}$

\footnotetext{
17 Taylor, J. E.; Jones, M. D.; Williams, J. M. J.; Bull, S. D. Org. Lett. 2010, 12, 5740-5743.
} 
<smiles>Cn1cc(C(=O)c2ccccc2NC(F)(F)F)c2ccccc21</smiles>

$8 \mathbf{a}$

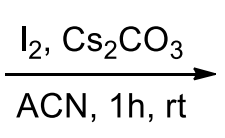<smiles>Cn1c2ccccc2c2c(=O)c3ccccc3n([As])c21</smiles>

$12 a$

Following a reported procedure, ${ }^{18} \mathbf{8 a}\left(36.4 \mathrm{mg}, 90.0 \mu \mathrm{mol}, 1.00\right.$ equiv.) and $\mathrm{Cs}_{2} \mathrm{CO}_{3}(58.7 \mathrm{mg}, 180 \mu \mathrm{mol}$, 2.00 equiv.) were suspended in $\mathrm{ACN}(9 \mathrm{~mL}) . \mathrm{I}_{2}(45.7 \mathrm{mg}, 180 \mu \mathrm{mol}, 2.00$ equiv.) was added, the mixture was stirred $1 \mathrm{~h}$ at $\mathrm{rt}$ and then quenched with a saturated aqueous $\mathrm{Na}_{2} \mathrm{~S}_{2} \mathrm{O}_{3}$ solution. The layers were separated and the aqueous layer was extracted three times with EtOAc $(10 \mathrm{~mL})$. The combined organic layers were washed with brine, dried over $\mathrm{MgSO}_{4}$ and concentrated under reduced pressure. A purification by column chromatography on silica gel (Pentane/EtOAc 4:1) afforded the title compound 12a (36.2 mg, $90.0 \mu \mathrm{mol}$, $100 \%$ yield) as a white solid. mp $161-163{ }^{\circ} \mathrm{C}$. $\mathbf{R f} 0.85$ (Pentane/EtOAc 1:1).

${ }^{1} \mathbf{H}$ NMR $\left(400 \mathrm{MHz}, \mathrm{CDCl}_{3}\right) \delta 8.29\left(\mathrm{~d}, J=7.7 \mathrm{~Hz}, 1 \mathrm{H}, \underline{\mathrm{H}}_{\mathrm{Ar}}\right), 8.17\left(\mathrm{~d}, J=8.2 \mathrm{~Hz}, 1 \mathrm{H}, \underline{\mathrm{H}}_{\mathrm{Ar}}\right), 7.99(\mathrm{dd}, J=7.8$, $\left.1.5 \mathrm{~Hz}, 1 \mathrm{H}, \underline{\mathrm{H}}_{\mathrm{Ar}}\right), 7.60\left(\mathrm{td}, J=8.3,7.9,1.6 \mathrm{~Hz}, 1 \mathrm{H}, \underline{\mathrm{H}}_{\mathrm{Ar}}\right), 7.50\left(\mathrm{~d}, J=8.0 \mathrm{~Hz}, 1 \mathrm{H}, \underline{\mathrm{H}}_{\mathrm{Ar}}\right), 7.48-7.36(\mathrm{~m}, 3 \mathrm{H}$, $\left.\underline{\mathrm{H}}_{\mathrm{Ar}}\right), 6.91\left(\mathrm{~d}, J=8.2 \mathrm{~Hz}, 2 \mathrm{H}, \underline{\mathrm{H}}_{\mathrm{Ar}}\right), 6.81\left(\mathrm{~d}, J=8.4 \mathrm{~Hz}, 2 \mathrm{H}, \underline{\mathrm{H}}_{\mathrm{Ar}}\right), 4.10\left(\mathrm{~s}, 3 \mathrm{H}, \mathrm{NC} \underline{\mathrm{H}}_{3}\right), 2.26$ (s, 3H, $\left.\underline{\mathrm{H}}_{3}\right)$.

${ }^{13} \mathbf{C}$ NMR $\left(101 \mathrm{MHz}, \mathrm{CDCl}_{3}\right) \delta 175.9,146.0,144.4,139.2,136.7,131.1,129.2,128.9,128.2,127.6,126.4$, $125.8,124.6,123.2,122.6,121.9,110.3,109.2,33.3,21.8$. One carbon atom is not resolved.

IR $v_{\max } 3056(\mathrm{w}), 1640(\mathrm{~m}), 1596(\mathrm{w}), 1524(\mathrm{w}), 1486(\mathrm{~m}), 1461(\mathrm{~m}), 1448(\mathrm{w}), 1417(\mathrm{w}), 1370(\mathrm{~m}), 1263$ (m), $1169(\mathrm{~m}), 780(\mathrm{~m}), 736(\mathrm{~s})$.

HRMS calculated for $\mathrm{C}_{23} \mathrm{H}_{19} \mathrm{~N}_{2} \mathrm{O}_{3} \mathrm{~S}^{+}[\mathrm{M}+\mathrm{H}]^{+} 403.1111$; found 403.1112 .

6-Methyl-5-((4-nitrophenyl)sulfonyl)-5,6-dihydro-11H-indolo[2,3b]quinolin-11-one (12b)<smiles>Cn1cc(C(=O)c2ccccc2NS(C)(=O)=O)c2ccccc21</smiles>

$8 \mathbf{b}$

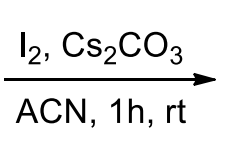<smiles>Cn1c2ccccc2c2c(=O)c3ccccc3n(S(C)(=O)=O)c21</smiles>

$12 b$

Following the same procedure as described for 12a, starting from $\mathbf{8 b}$ (87.0 $\mathrm{mg}, 0.200 \mathrm{mmol}, 1.00$ equiv.), $\mathrm{Cs}_{2} \mathrm{CO}_{3}$ (130 mg, $0.400 \mathrm{mmol}, 2.00$ equiv.) and $\mathrm{I}_{2}$ (101 mg, $0.400 \mathrm{mmol}, 2.00$ equiv.). A purification by column chromatography on silica gel (Pentane/EtOAc 4:1) afforded the title compound $\mathbf{1 2 b}$ (77.0 mg, 0.178 mmol, $89 \%$ yield) as a yellow solid. $\mathbf{m p ~} 187-189{ }^{\circ} \mathrm{C}$. Rf 0.89 (Pentane/EtOAc 1:1).

1H NMR (400 MHz, CDCl $\left.{ }_{3}\right) \delta 8.27\left(\mathrm{~d}, J=7.8 \mathrm{~Hz}, 1 \mathrm{H}, \underline{\mathrm{H}}_{\mathrm{Ar}}\right), 8.17\left(\mathrm{~d}, J=7.9 \mathrm{~Hz}, 1 \mathrm{H}, \underline{\mathrm{H}}_{\mathrm{Ar}}\right), 8.00(\mathrm{dd}, J=$ 7.8, $\left.1.6 \mathrm{~Hz}, 1 \mathrm{H}, \underline{\mathrm{H}}_{\mathrm{Ar}}\right), 7.99-7.95\left(\mathrm{~m}, 2 \mathrm{H}, \underline{\mathrm{H}}_{\mathrm{Ar}}\right), 7.66\left(\mathrm{td}, J=8.1,1.6 \mathrm{~Hz}, 1 \mathrm{H}, \underline{\mathrm{H}}_{\mathrm{Ar}}\right), 7.54-7.45\left(\mathrm{~m}, 3 \mathrm{H}, \underline{\mathrm{H}}_{\mathrm{Ar}}\right)$, $7.42\left(\mathrm{td}, J=7.6,1.3 \mathrm{~Hz}, 1 \mathrm{H}, \underline{\mathrm{H}}_{\mathrm{Ar}}\right), 7.15-7.09\left(\mathrm{~m}, 2 \mathrm{H}, \underline{\mathrm{H}}_{\mathrm{Ar}}\right), 4.11\left(\mathrm{~s}, 3 \mathrm{H}, \mathrm{NC}_{\underline{H}_{3}}\right)$.

${ }^{13} \mathrm{C}$ NMR $\left(101 \mathrm{MHz}, \mathrm{CDCl}_{3}\right) \delta 175.4,151.0,143.3,138.3,137.2,136.7,131.6,131.1,129.6,128.4$, $126.9,125.9,125.2,123.7,123.6,122.4,122.1,110.5,109.3,33.3$.

IR $v_{\max } 3105$ (w), 3062 (w), 1646 (s), 1601 (m), 1532 (s), 1491 (s), 1461 (s), 1379 (m), 1350 (s), 1186 (s), 1171 (s), 784 (s), 738 (s).

HRMS calculated for $\mathrm{C}_{22} \mathrm{H}_{15} \mathrm{~N}_{3} \mathrm{NaO}_{5} \mathrm{~S}^{+}[\mathrm{M}+\mathrm{Na}]^{+} 456.0625$; found 456.0630.

${ }^{18}$ Li, Y.-X.; Wang, H.-X.; Ali, S.; Xia, X.-F.; Liang, Y.-M. Chem. Commun. 2012, 48, 2343-2345. 
<smiles>Cn1c2ccccc2c2c(=O)c3ccccc3n(S(C)(=O)=O)c21</smiles>

$12 \mathrm{~b}$

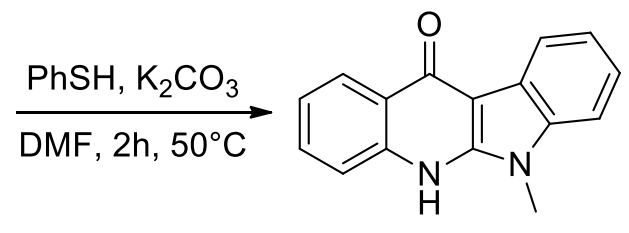

13

12b (65.0 mg, $0.150 \mathrm{mmol}, 1.00$ equiv.), $\mathrm{K}_{2} \mathrm{CO}_{3}$ (124 mg, $0.900 \mathrm{mmol}, 6.00$ equiv.) and benzenethiol (61 $\mu \mathrm{L}, 0.60 \mathrm{mmol}, 4.0$ equiv.) were solubilized in DMF $(1.5 \mathrm{~mL})$. The mixture was stirred $2 \mathrm{~h}$ at $50{ }^{\circ} \mathrm{C}$ and then filtered over Celite with $\mathrm{MeOH}(20 \mathrm{~mL})$. A purification by column chromatography on silica gel (DCM 100\% to DCM/MeOH 4:1) afforded the title compound 13 (32.7 mg, $0.132 \mathrm{mmol}, 88 \%$ yield) as a white solid. mp > $350{ }^{\circ} \mathrm{C}$. $\mathbf{R f} 0.34(\mathrm{DCM} / \mathrm{MeOH} 19: 1)$.

${ }^{1}$ H NMR (400 MHz, DMSO-d6) $\delta 12.33(\mathrm{~s}, 1 \mathrm{H}, \mathrm{N} \underline{\mathrm{H}}), 8.28\left(\mathrm{~d}, J=7.3 \mathrm{~Hz}, 1 \mathrm{H}, \underline{\mathrm{H}}_{\mathrm{Ar}}\right), 8.21(\mathrm{~d}, J=7.4 \mathrm{~Hz}$, $\left.1 \mathrm{H}, \underline{\mathrm{H}}_{\mathrm{Ar}}\right), 7.82\left(\mathrm{~d}, J=8.2 \mathrm{~Hz}, 1 \mathrm{H}, \underline{\mathrm{H}}_{\mathrm{Ar}}\right), 7.66\left(\mathrm{t}, J=7.0 \mathrm{~Hz}, 1 \mathrm{H}, \underline{\mathrm{H}}_{\mathrm{Ar}}\right), 7.53\left(\mathrm{~d}, J=7.9 \mathrm{~Hz}, 1 \mathrm{H}, \underline{\mathrm{H}}_{\mathrm{Ar}}\right), 7.37-$ $7.28\left(\mathrm{~m}, 2 \mathrm{H}, \underline{\mathrm{H}}_{\mathrm{Ar}}\right), 7.24\left(\mathrm{t}, J=7.2 \mathrm{~Hz}, 1 \mathrm{H}, \underline{\mathrm{H}}_{\mathrm{Ar}}\right), 3.95\left(\mathrm{~s}, 3 \mathrm{H}, \mathrm{NC}_{\underline{H}}\right)$.

${ }^{13}$ C NMR (101 MHz, DMSO-d6) $\delta$ 172.0, 145.9, 138.5, 136.2, 130.8, 125.3, 123.6, 123.3, 122.6, 121.7, 121.2, 120.0, 117.7, 109.1, 101.2, 29.3.

IR $v_{\max } 2994$ (br), 1639 (s), 1583 (s), 1535 (s), 1482 (s), 1465 (s), 740 (s).

HRMS calculated for $\mathrm{C}_{16} \mathrm{H}_{13} \mathrm{~N}_{2} \mathrm{O}^{+}[\mathrm{M}+\mathrm{H}]^{+} 249.1022$; found 249.1024.

\section{(2-Aminophenyl)(1-methyl-1H-indol-3-yl)methanone (14)}<smiles>Cn1cc(C(=O)c2ccccc2NS(N)(=O)=O)c2ccccc21</smiles>

8b

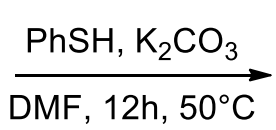<smiles>Cn1cc(C(=O)c2ccccc2N)c2ccccc21</smiles>

14

$\mathbf{8 b}$ (87.0 mg, $0.200 \mathrm{mmol}, 1.00$ equiv.), $\mathrm{K}_{2} \mathrm{CO}_{3}$ (166 mg, $1.20 \mathrm{mmol}, 6.00$ equiv.) and benzenethiol (82 $\mu \mathrm{L}$, $0.80 \mathrm{mmol}, 4.00$ equiv.) were solubilized in DMF $(2 \mathrm{~mL})$. The mixture was stirred overnight at $50{ }^{\circ} \mathrm{C}$. The mixture was diluted with water $(10 \mathrm{~mL})$. The layers were separated and the aqueous layer was extracted three times with EtOAc $(10 \mathrm{~mL})$. The combined organic layers were washed with brine, dried over $\mathrm{MgSO}_{4}$ and concentrated under reduced pressure. A purification by column chromatography on silica gel (Pentane/EtOAc 4:1) afforded the title compound $\mathbf{1 4}(50 \mathrm{mg}, 0.20 \mathrm{mmol}, 100 \%$ yield) as a yellow solid. mp 183-185 ${ }^{\circ} \mathrm{C}$. Rf 0.72 (Pentane/EtOAc 1:1).

${ }^{1} \mathbf{H}$ NMR $\left(400 \mathrm{MHz}, \mathrm{CDCl}_{3}\right) \delta 8.37-8.31\left(\mathrm{~m}, 1 \mathrm{H}, \underline{\mathrm{H}}_{\mathrm{Ar}}\right), 7.64\left(\mathrm{dd}, J=7.8,1.5 \mathrm{~Hz}, 1 \mathrm{H}, \underline{\mathrm{H}}_{\mathrm{Ar}}\right), 7.48(\mathrm{~s}, 1 \mathrm{H}$, $\mathrm{C} \underline{\mathrm{H}}-\mathrm{N}), 7.39-7.31\left(\mathrm{~m}, 3 \mathrm{H}, \underline{\mathrm{H}}_{\mathrm{Ar}}\right), 7.30-7.24\left(\mathrm{~m}, 1 \mathrm{H}, \underline{\mathrm{H}}_{\mathrm{Ar}}\right), 6.76-6.68\left(\mathrm{~m}, 2 \mathrm{H}, \underline{\mathrm{H}}_{\mathrm{Ar}}\right), 5.45\left(\mathrm{~s}, 2 \mathrm{H}, \mathrm{N}_{2}\right), 3.81$ (s, $\left.3 \overline{\mathrm{H}}, \mathrm{NCH}_{3}\right)$.

${ }^{13}$ C NMR $\left(101 \mathrm{MHz}, \mathrm{CDCl}_{3}\right) \delta 192.4,148.8,137.5,137.2,132.5,132.1,127.4,127.1,123.5,122.5,122.5$, $116.8,116.5,116.1,109.7,33.5$.

IR $v_{\max } 3672$ (br), 3475 (w), 3364 (w), 2976 (s), 2902 (s), 1610 (s), 1577 (m), 1521 (s), 1462 (m), 1394 (s), 1367 (s), 1230 (s), 1075 (s), 1053 (s), 888 (s), 744 (s).

HRMS calculated for $\mathrm{C}_{16} \mathrm{H}_{14} \mathrm{~N}_{2} \mathrm{NaO}^{+}[\mathrm{M}+\mathrm{Na}]^{+} 273.0998$; found 273.0999 . 


\section{Spectra of new compounds}
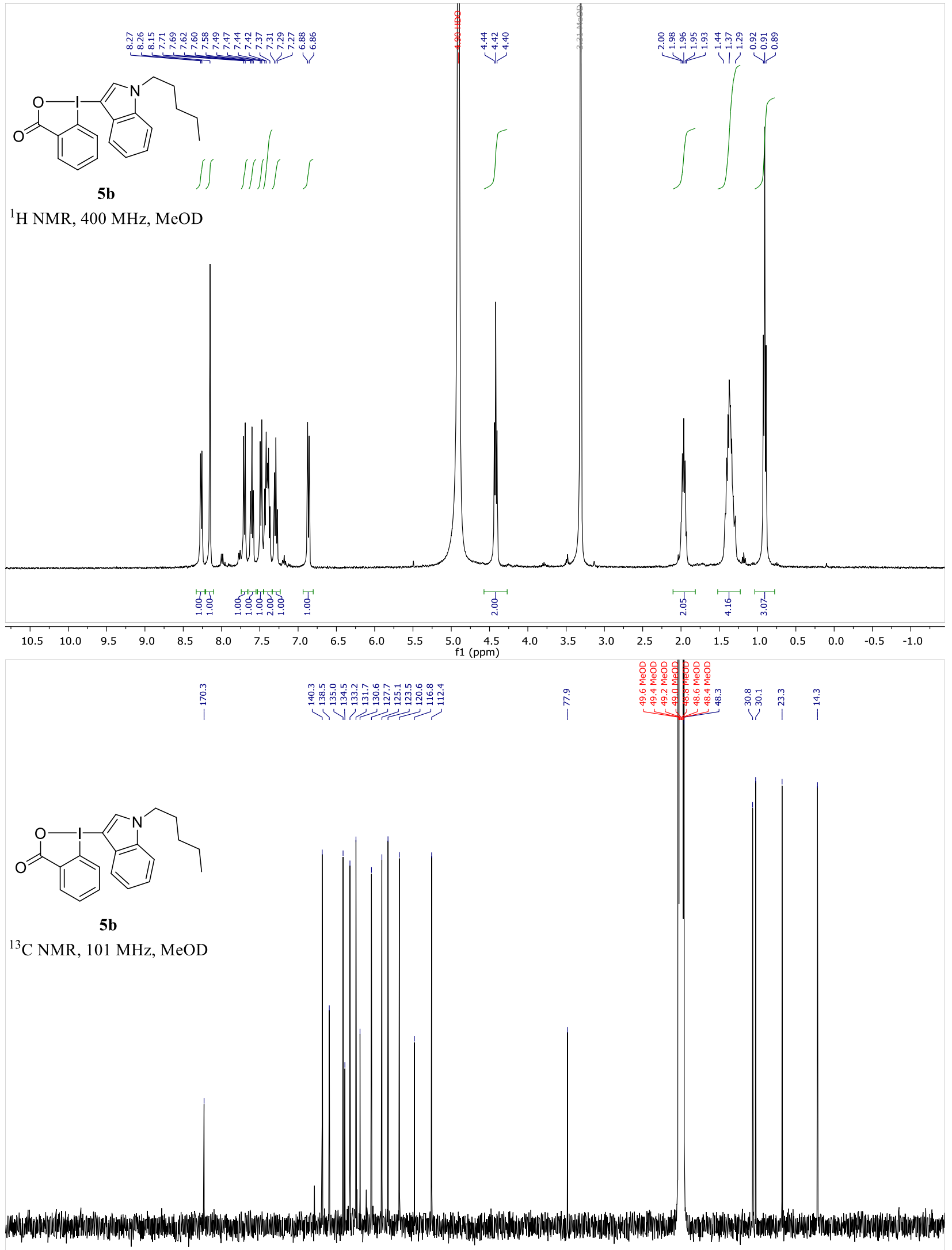

\begin{tabular}{llllllllllllllllllllllllll}
\hline 20 & 210 & 200 & 190 & 180 & 170 & 160 & 150 & 140 & 130 & 120 & 110 & 100 & 90 & 80 & 70 & 60 & 50 & 40 & 30 & 20 & 10 & 0 & -10
\end{tabular} 

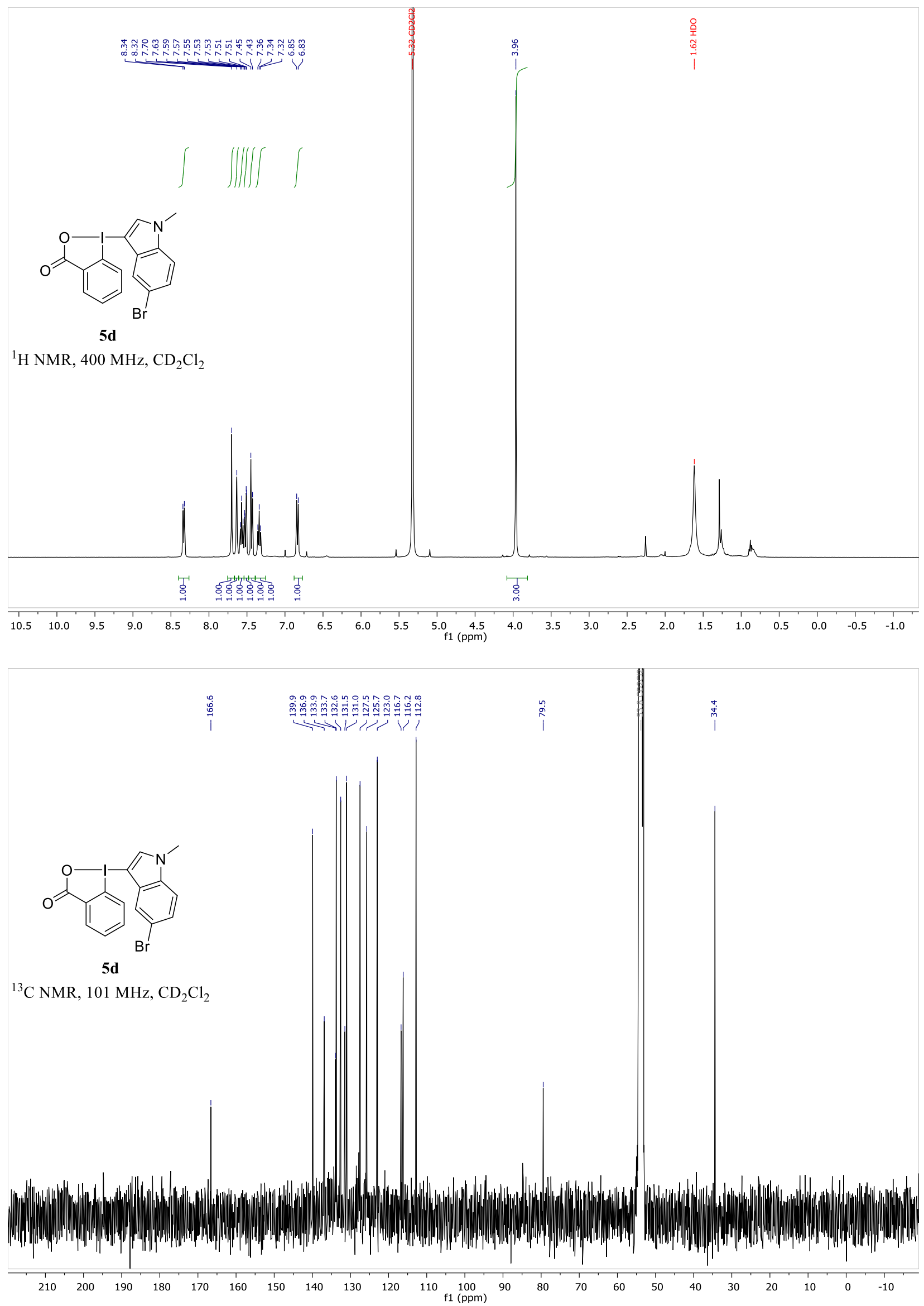

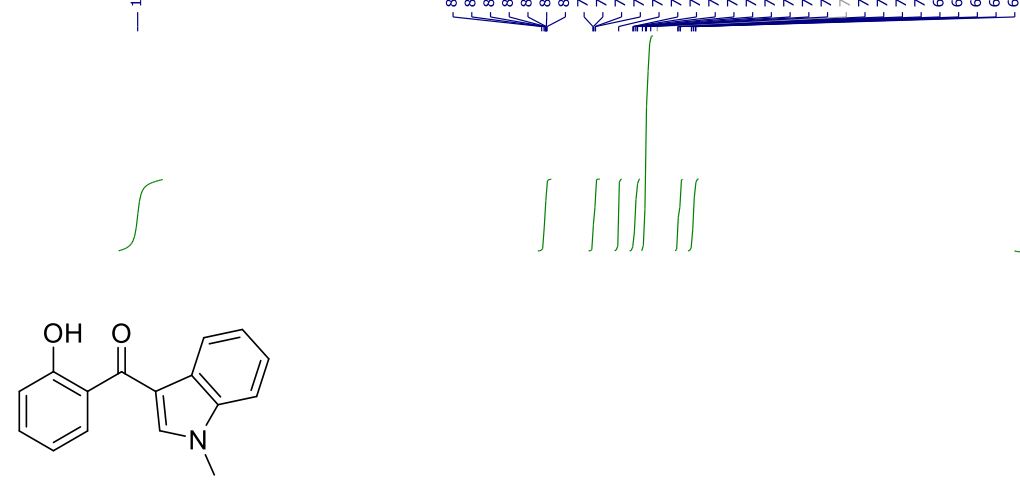

$6 \mathbf{a}$

${ }^{1} \mathrm{H}$ NMR, $400 \mathrm{MHz}, \mathrm{CDCl}_{3}$
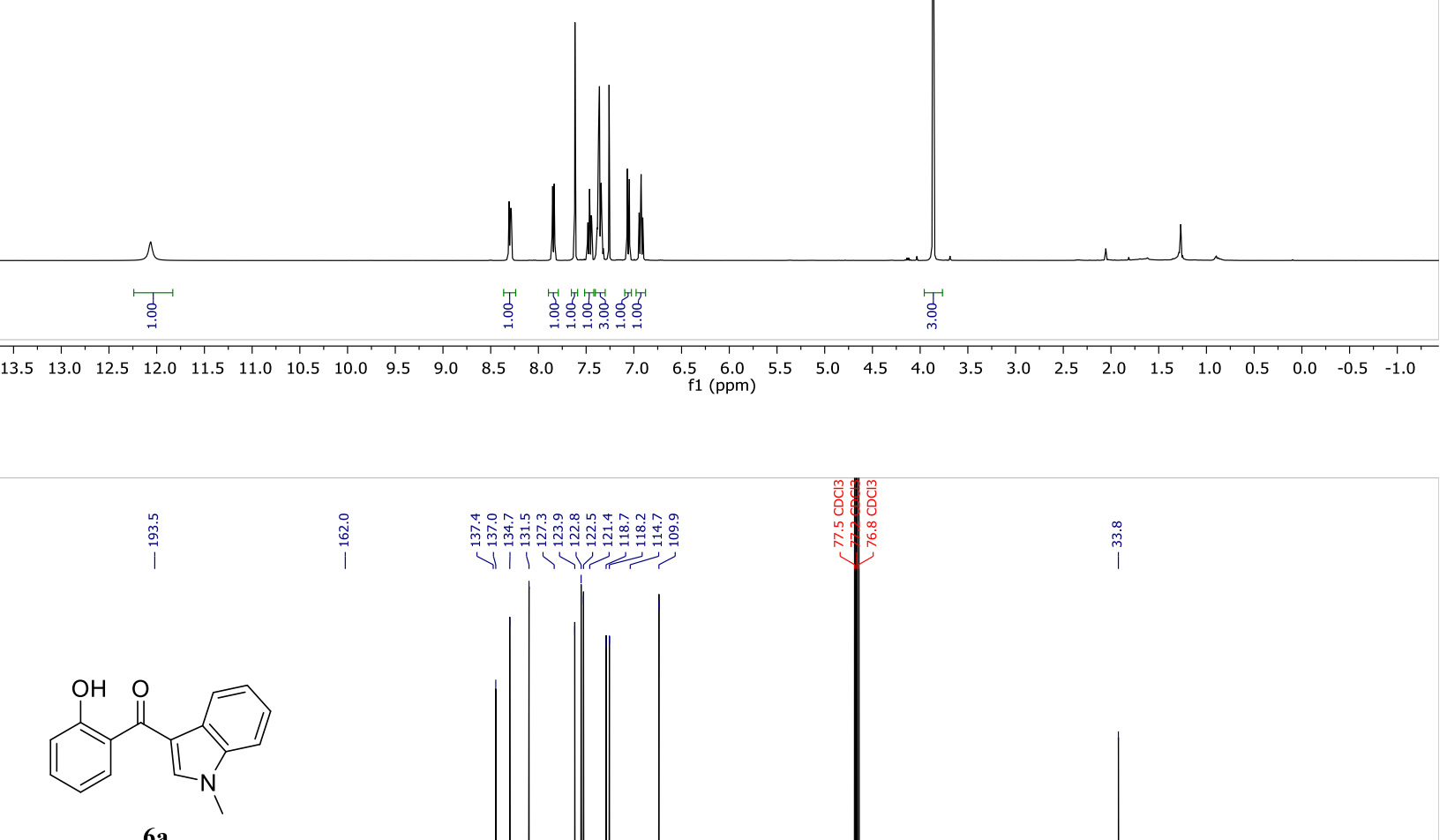

${ }^{13} \mathrm{C}$ NMR, $101 \mathrm{MHz}, \mathrm{CDCl}_{3}$
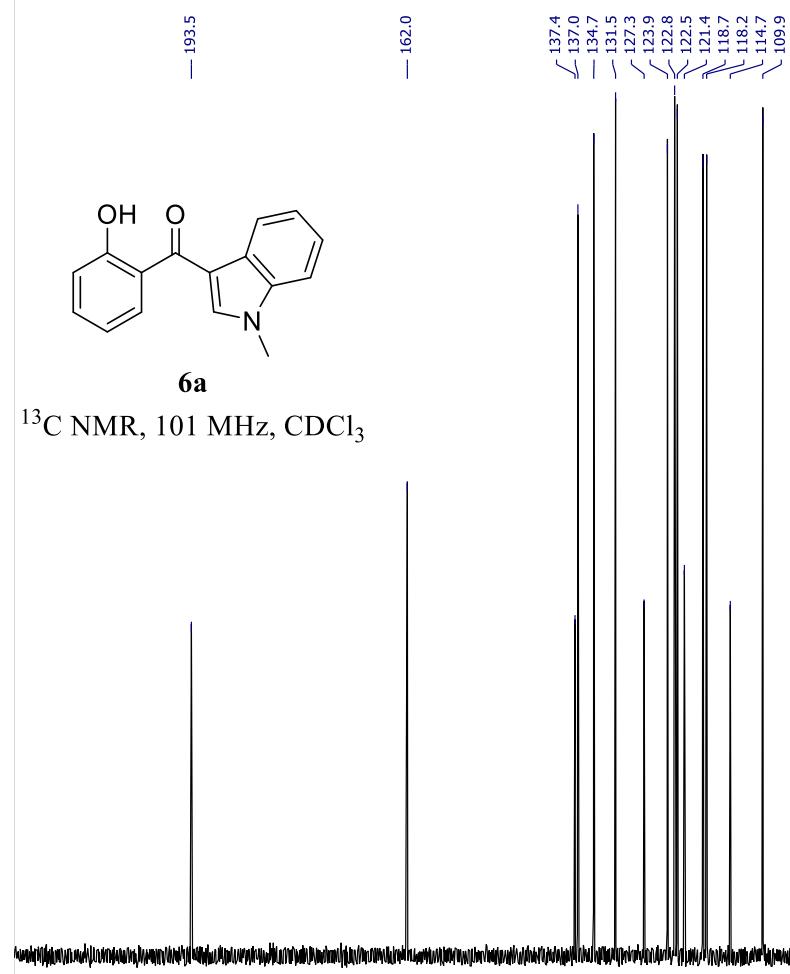

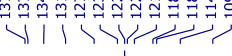

$\stackrel{\infty}{\dddot{m}}$

w

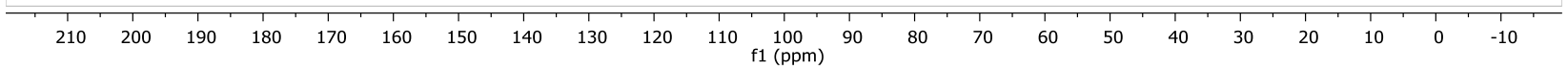



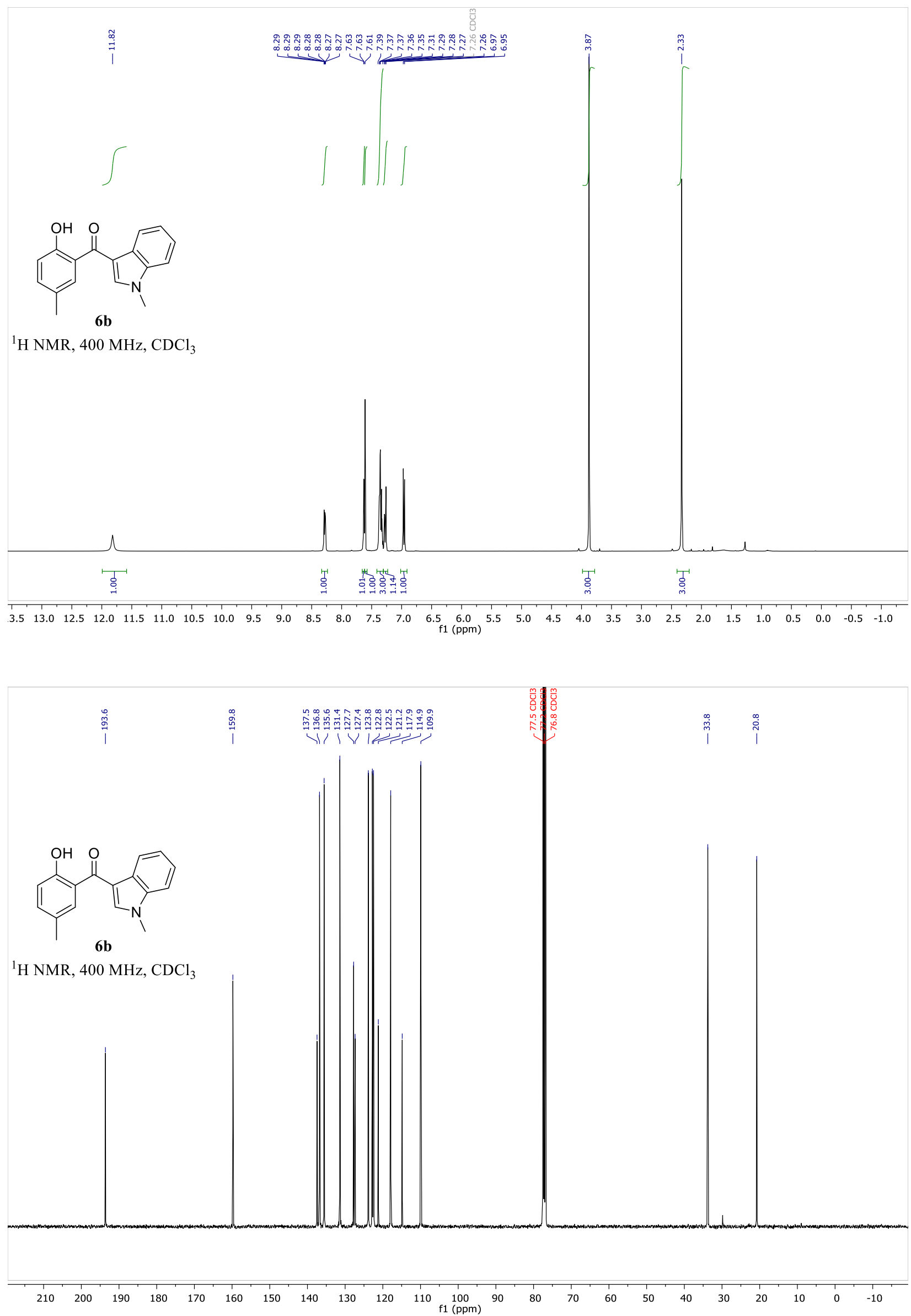

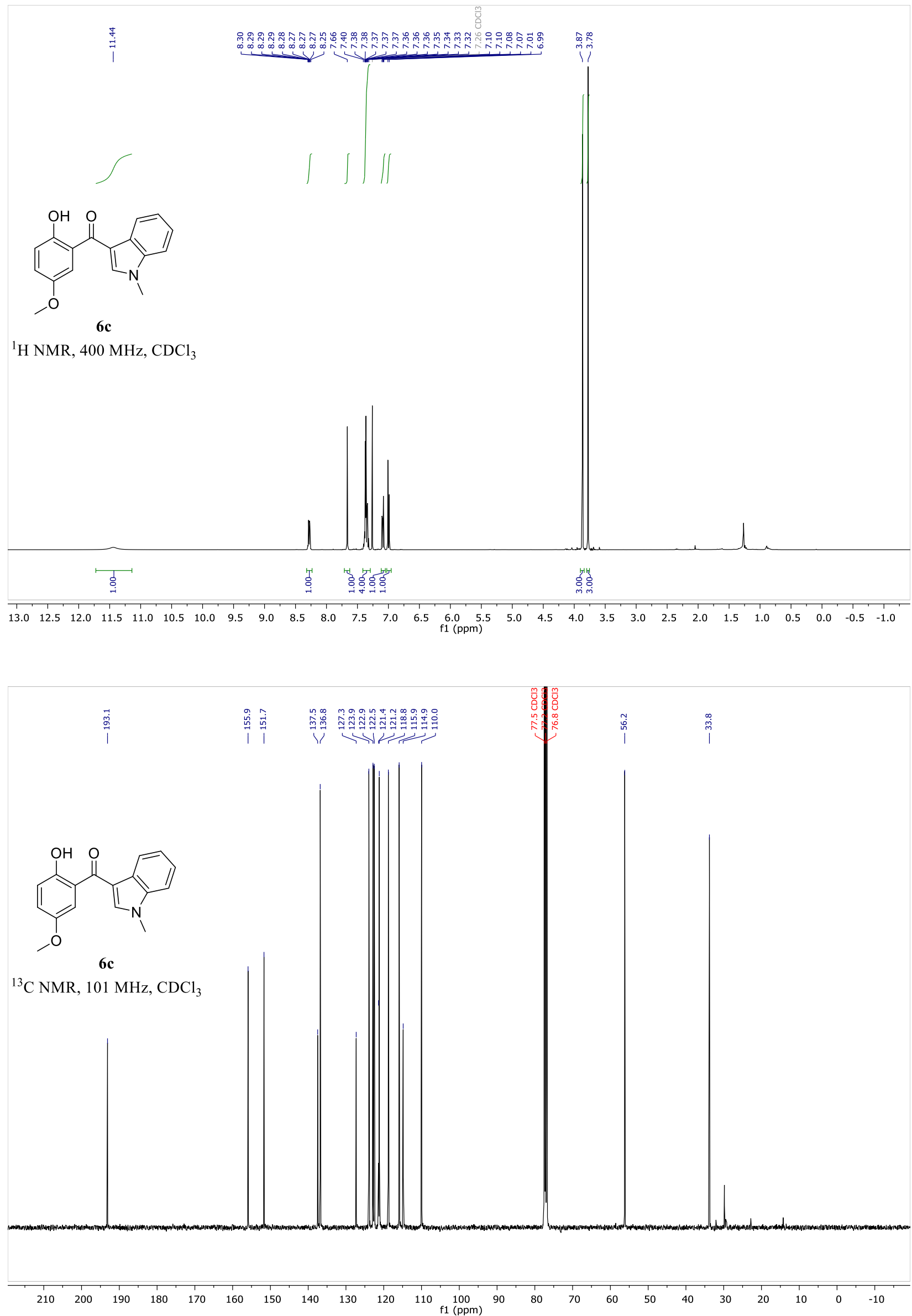

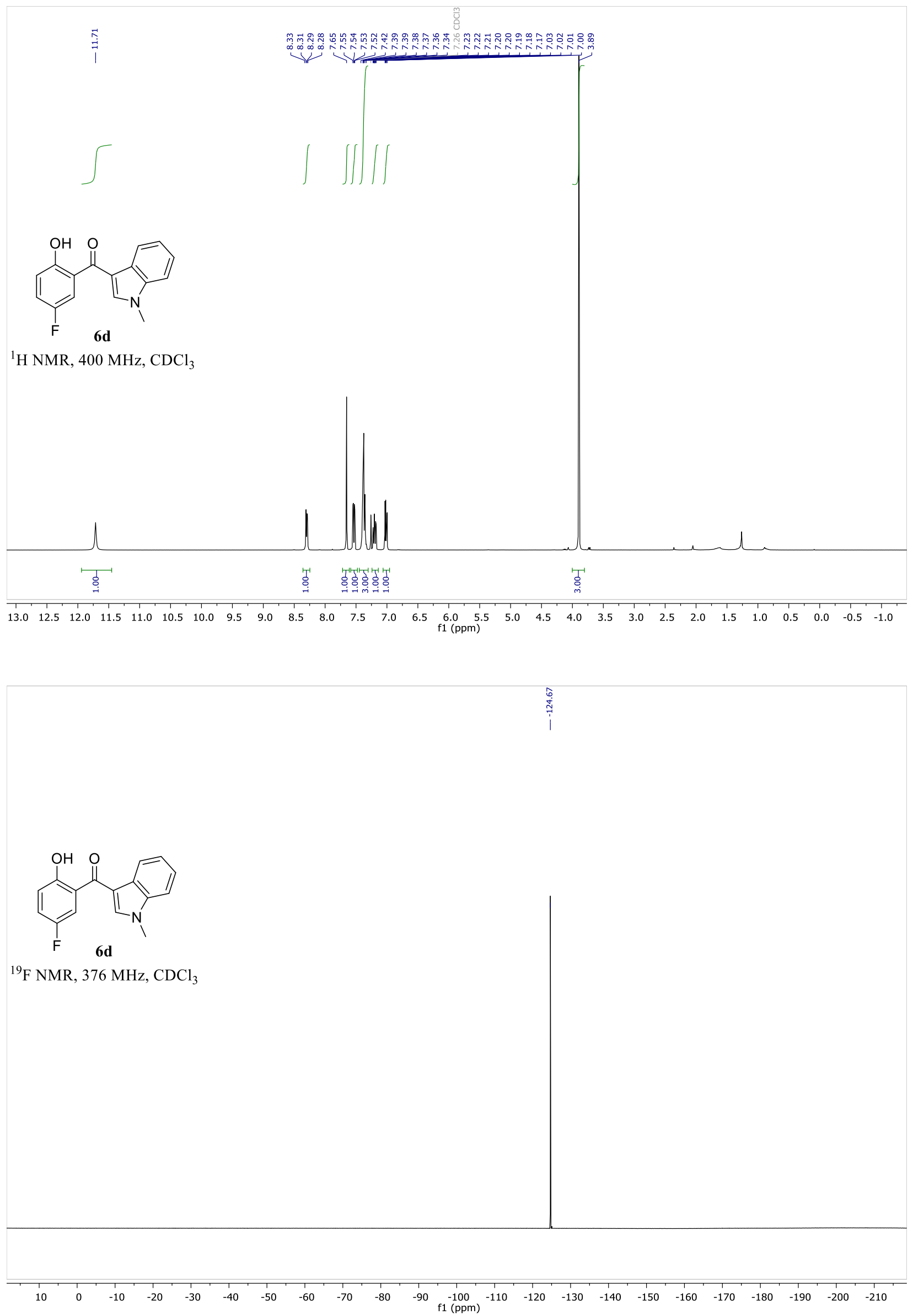


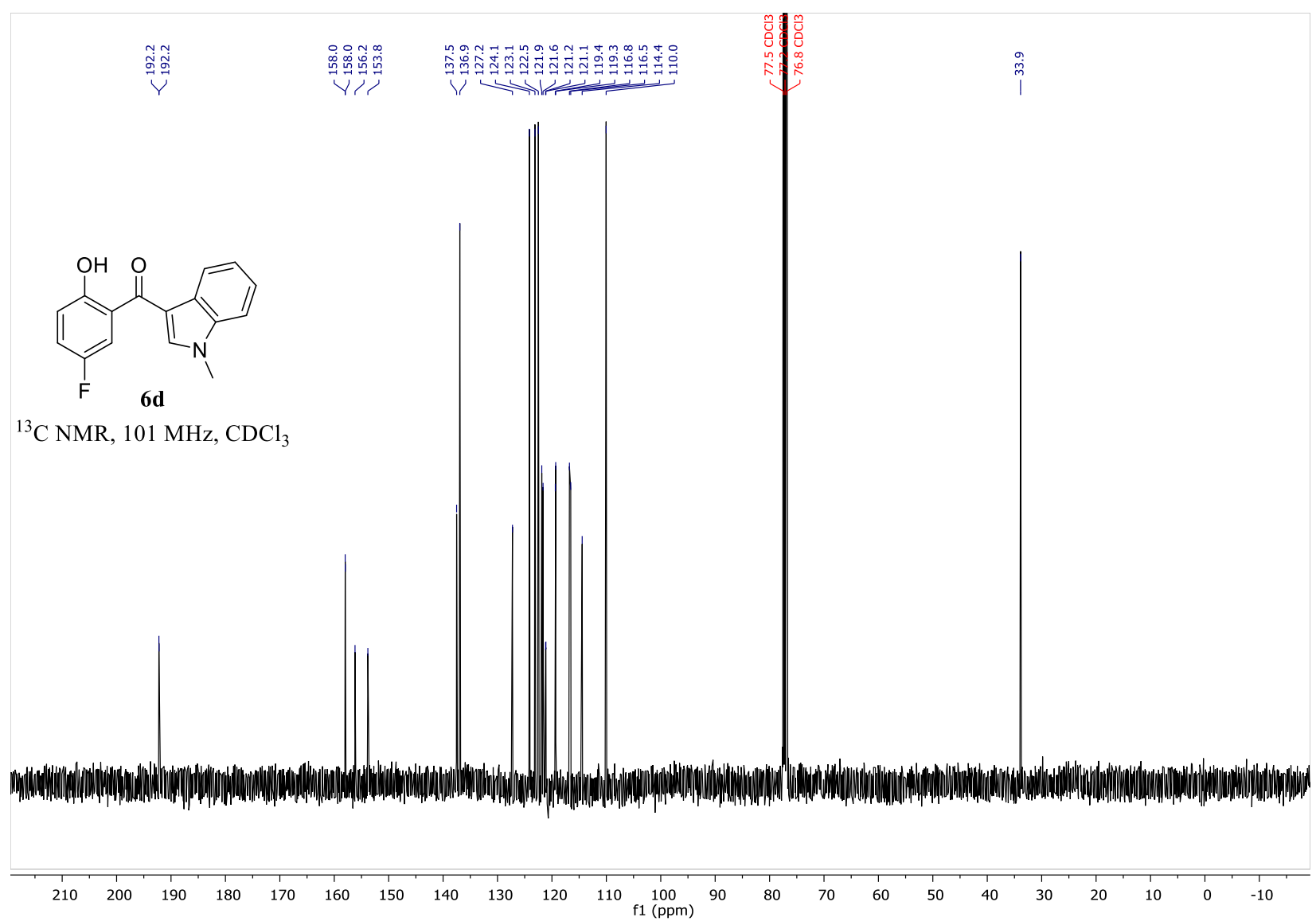



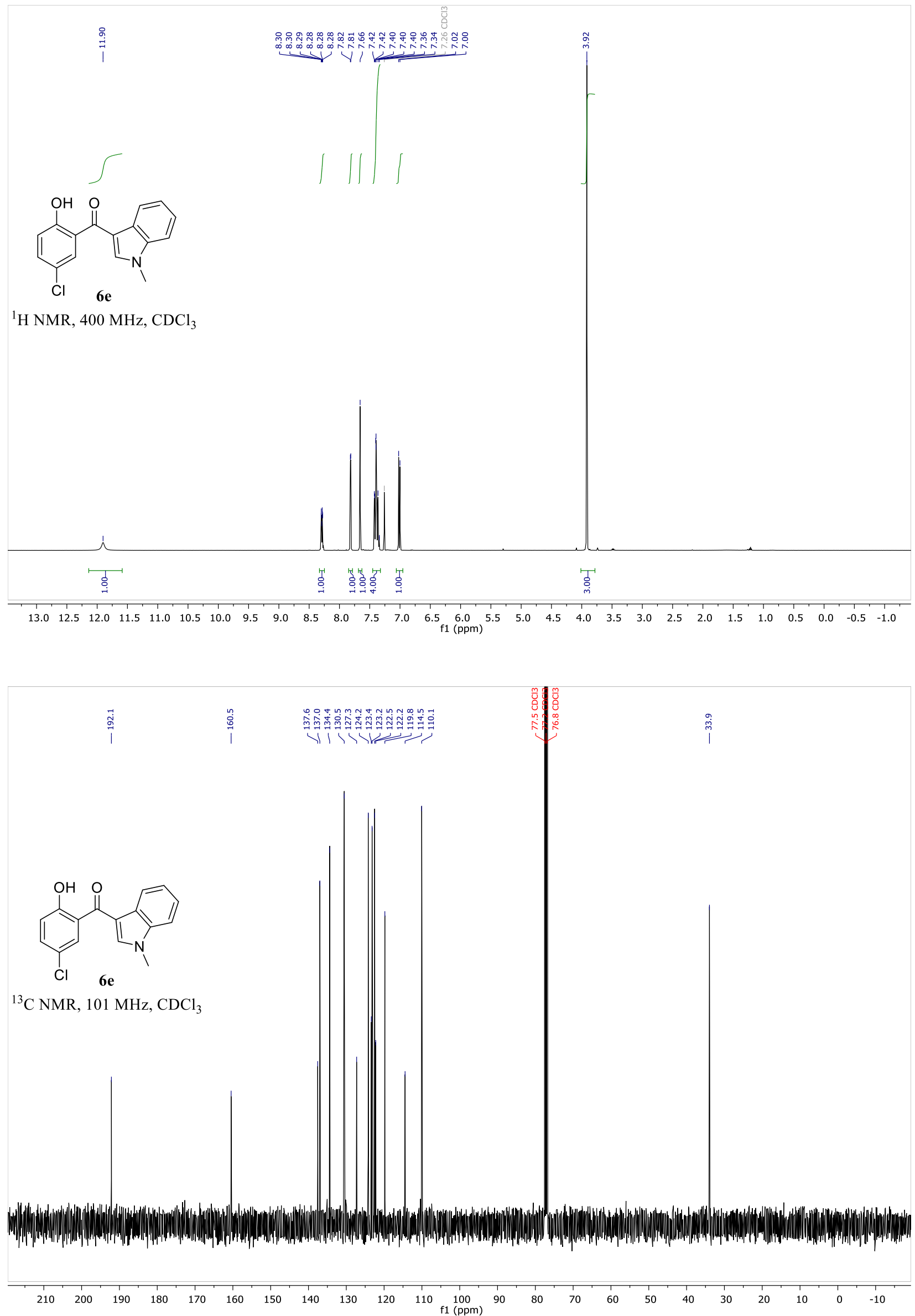

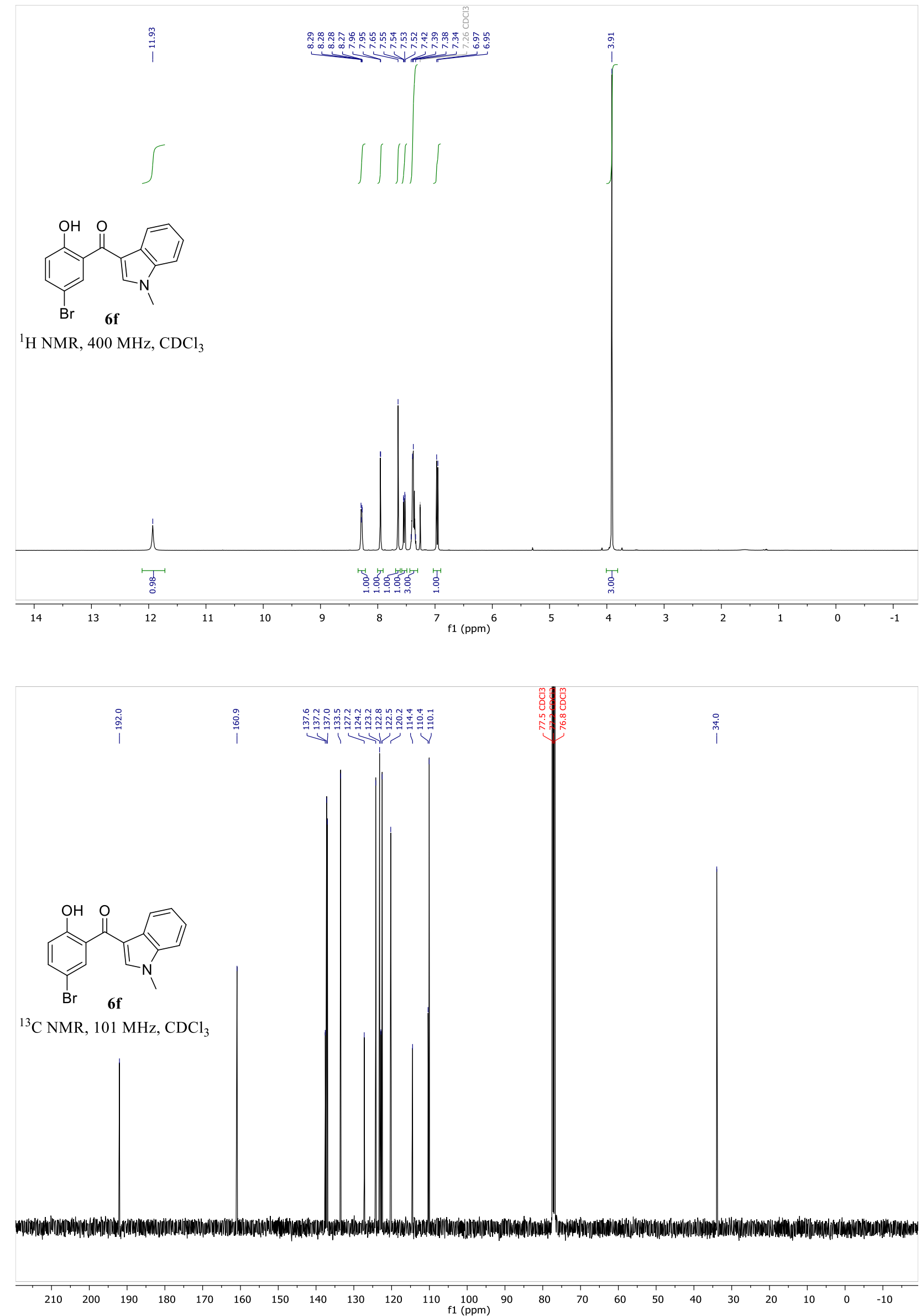

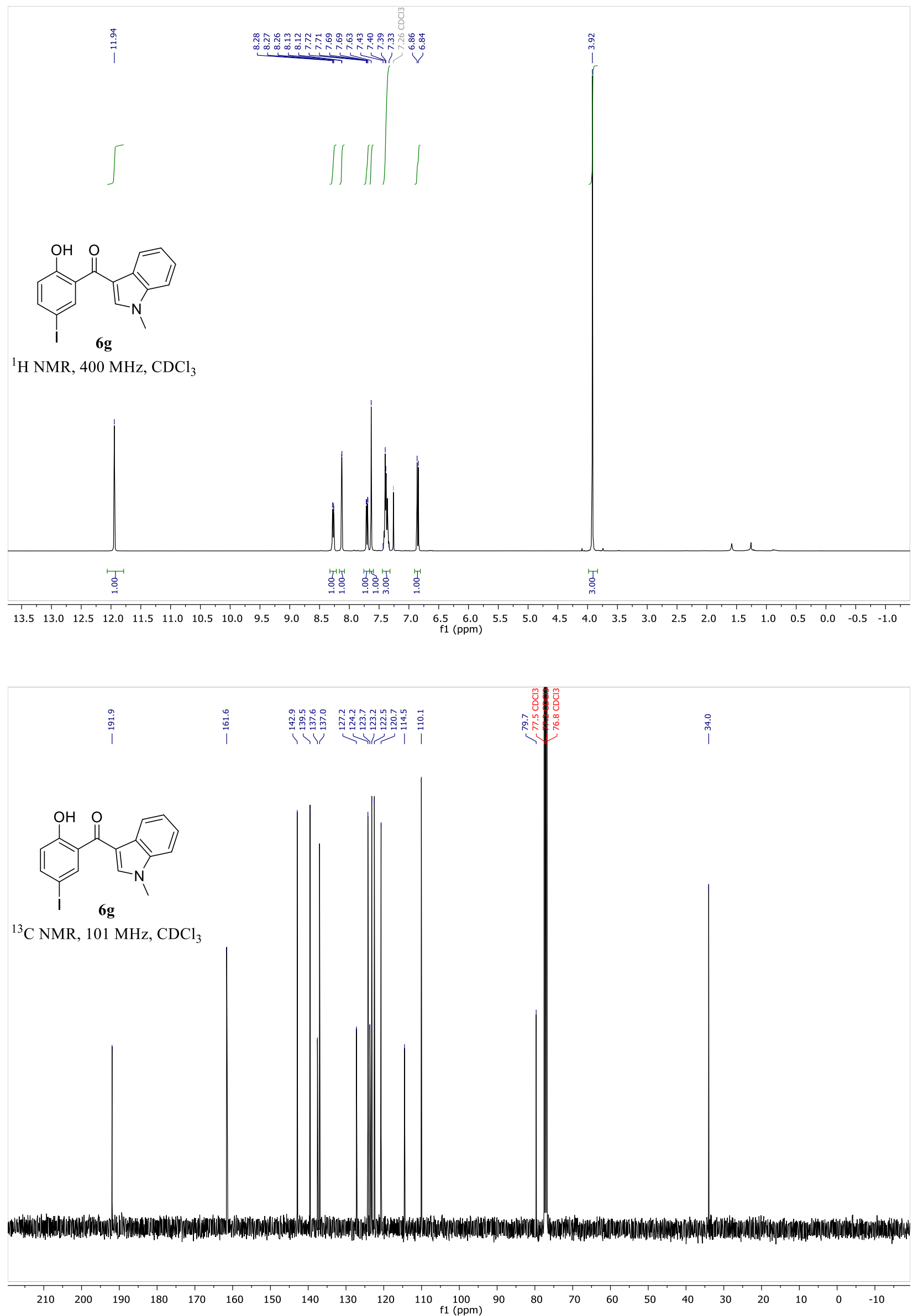

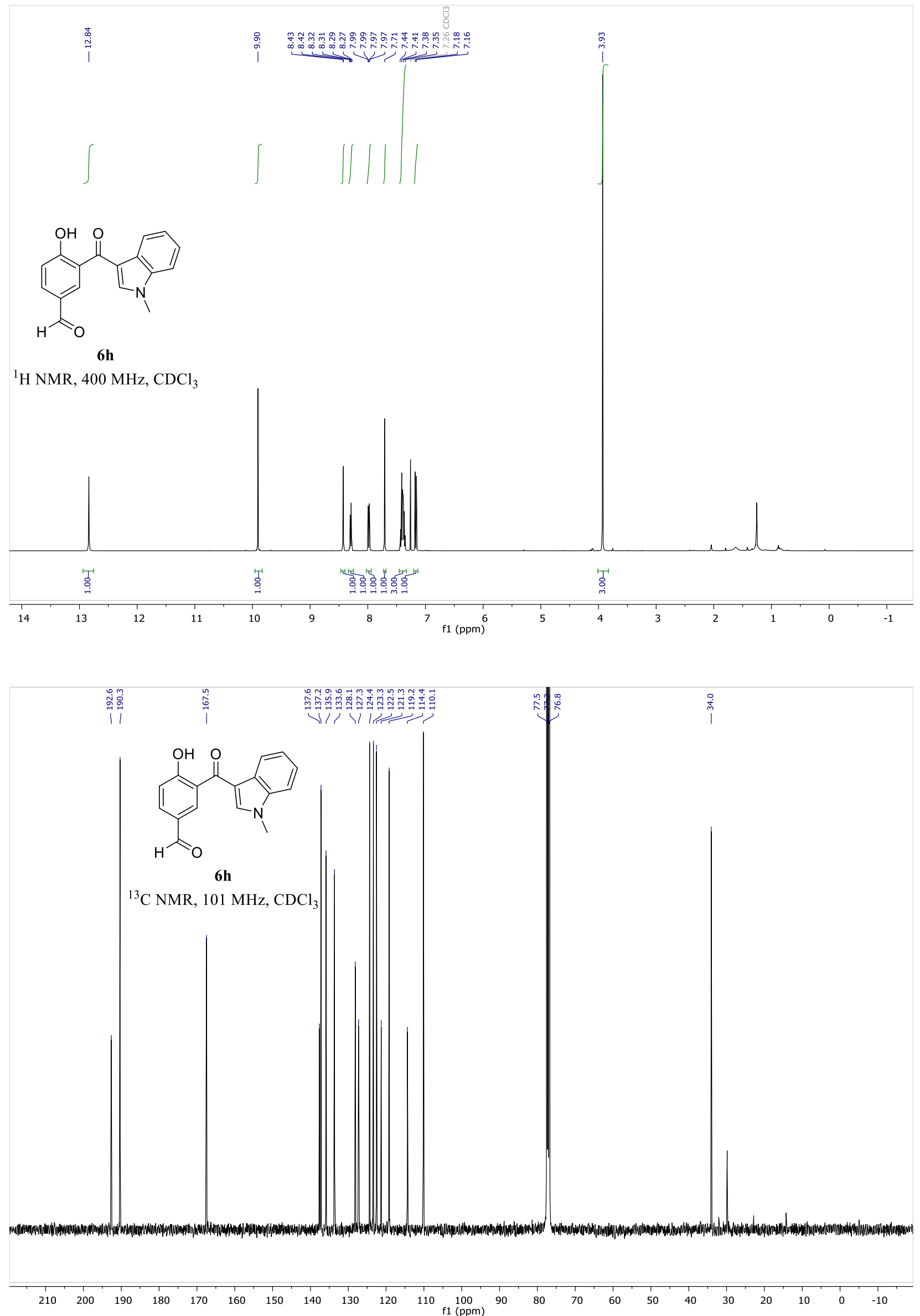

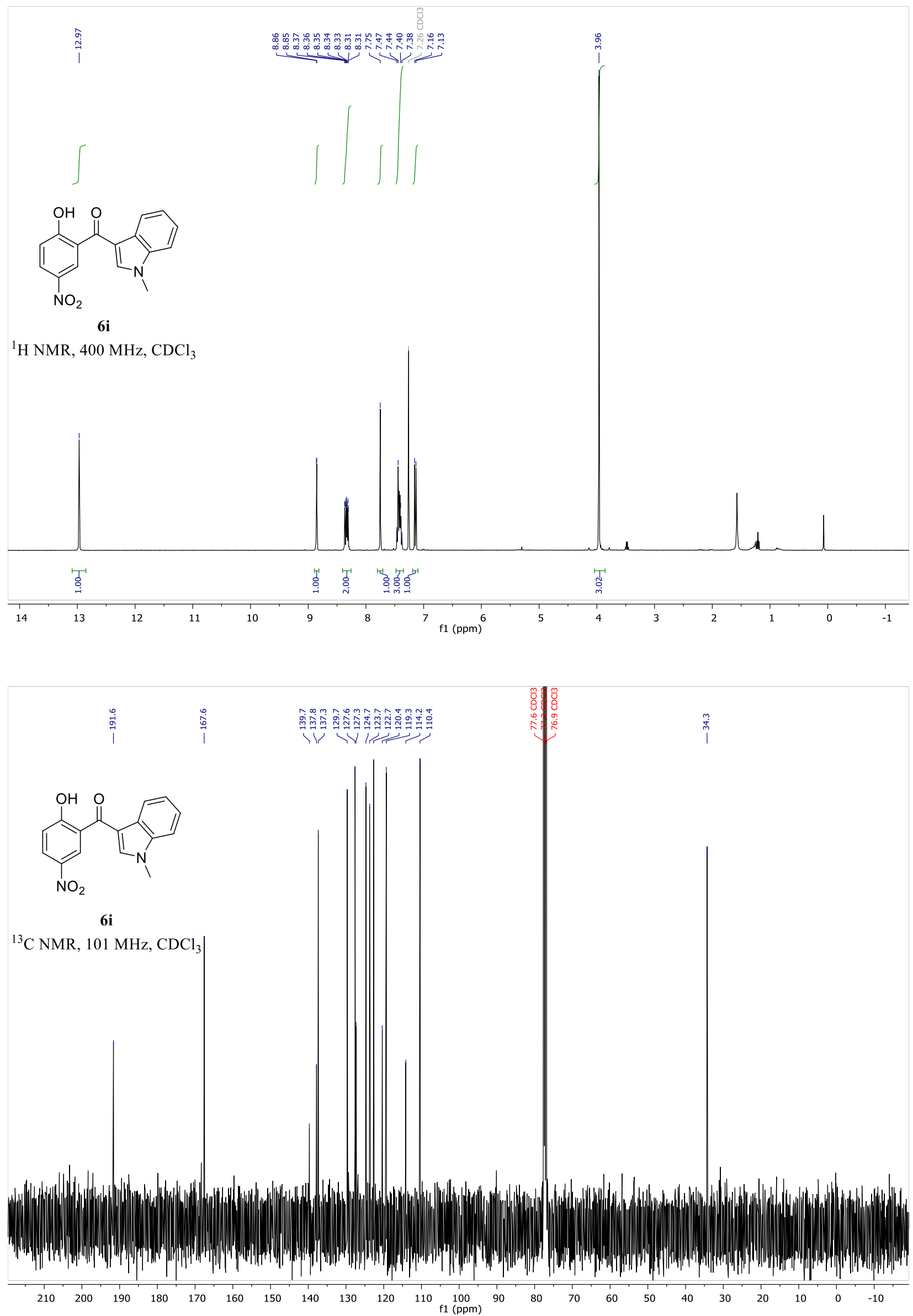

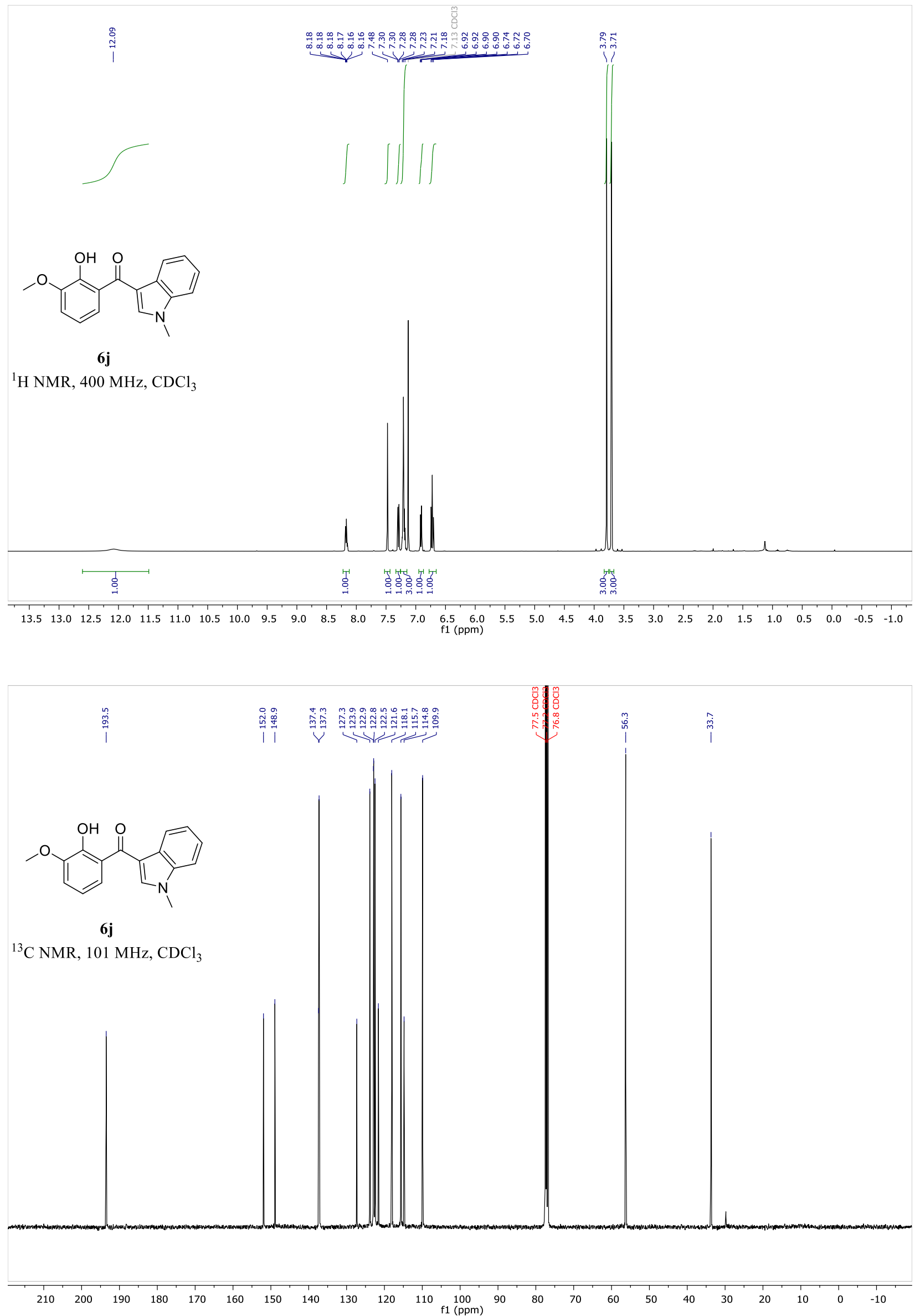

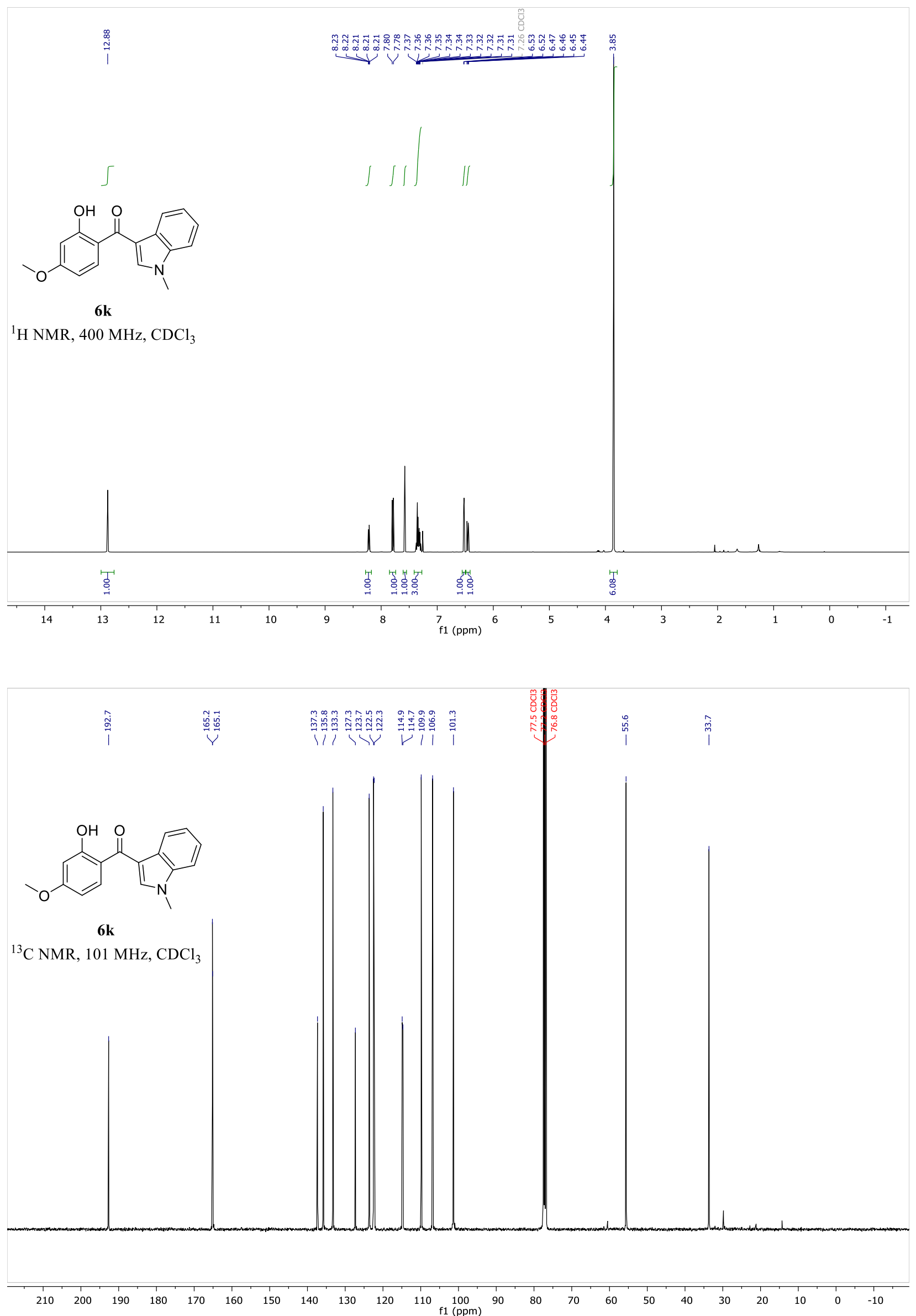

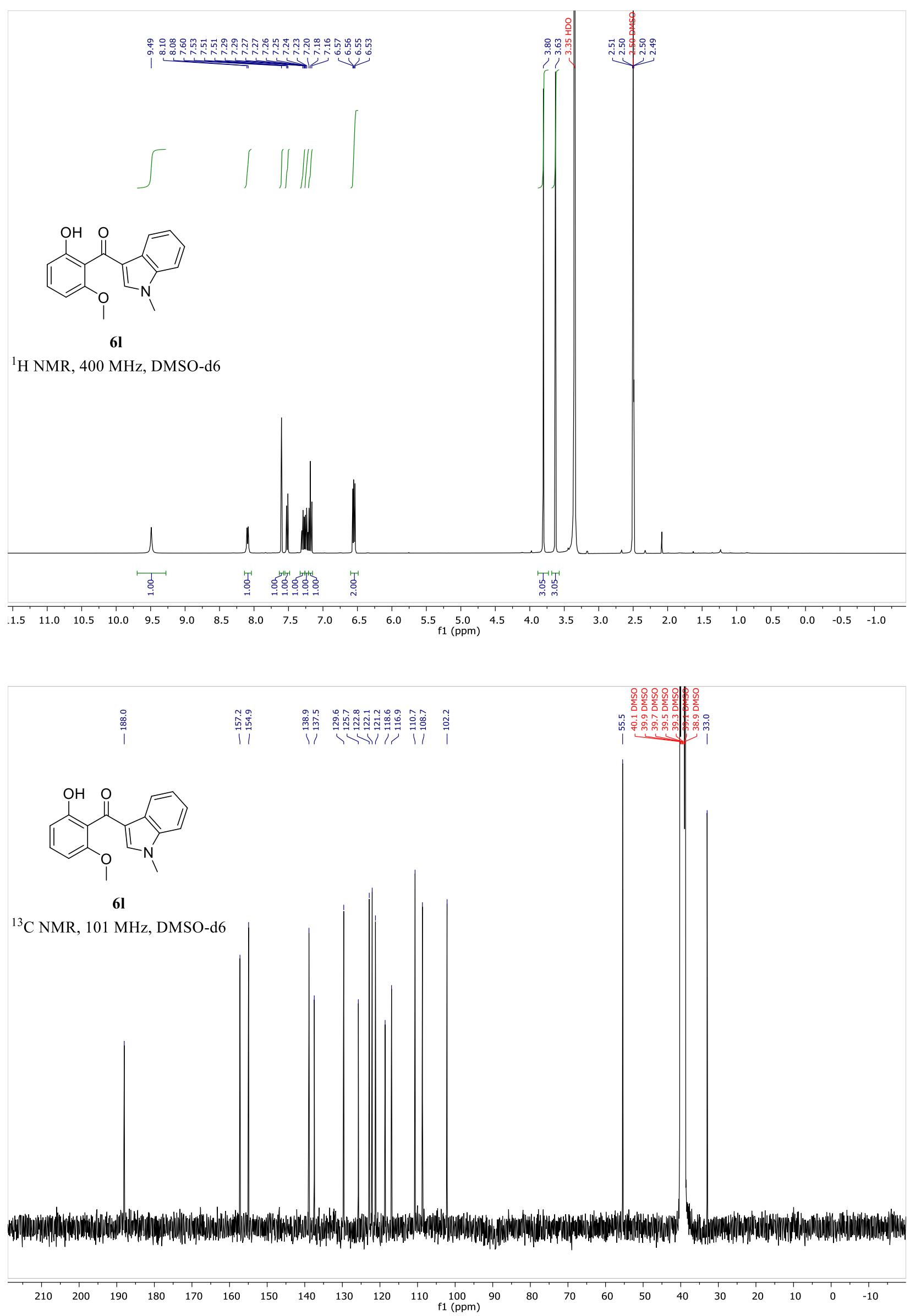

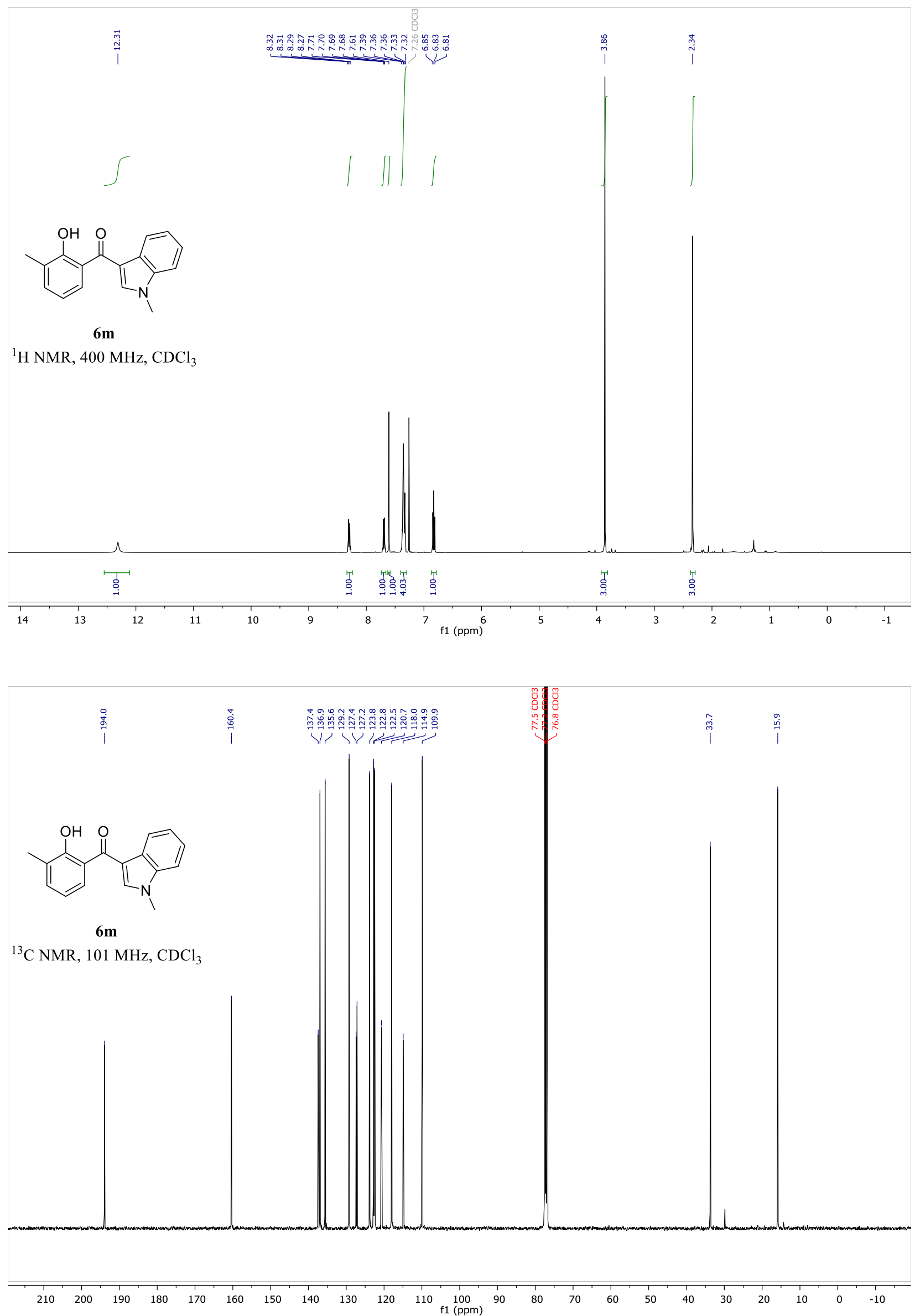

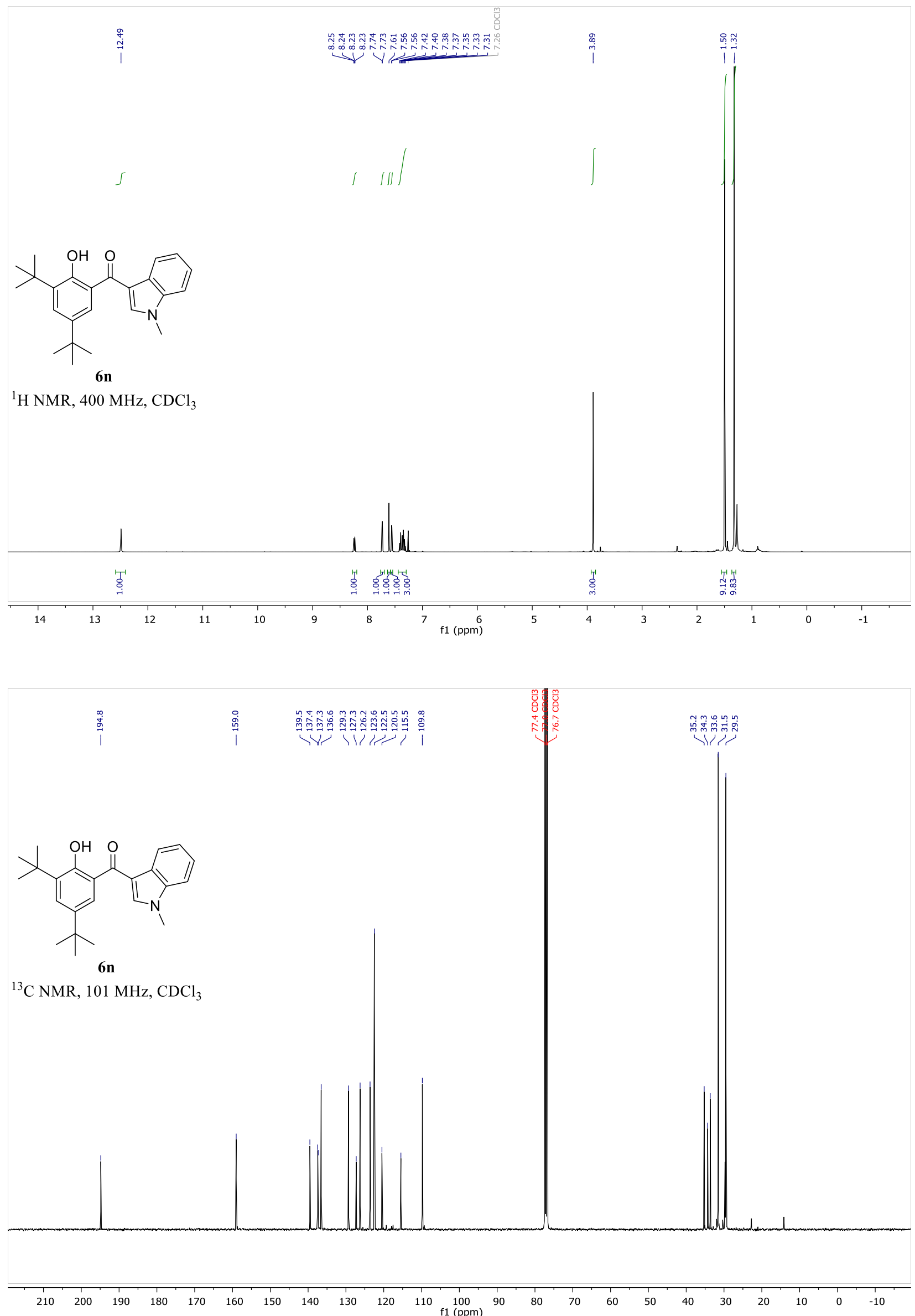

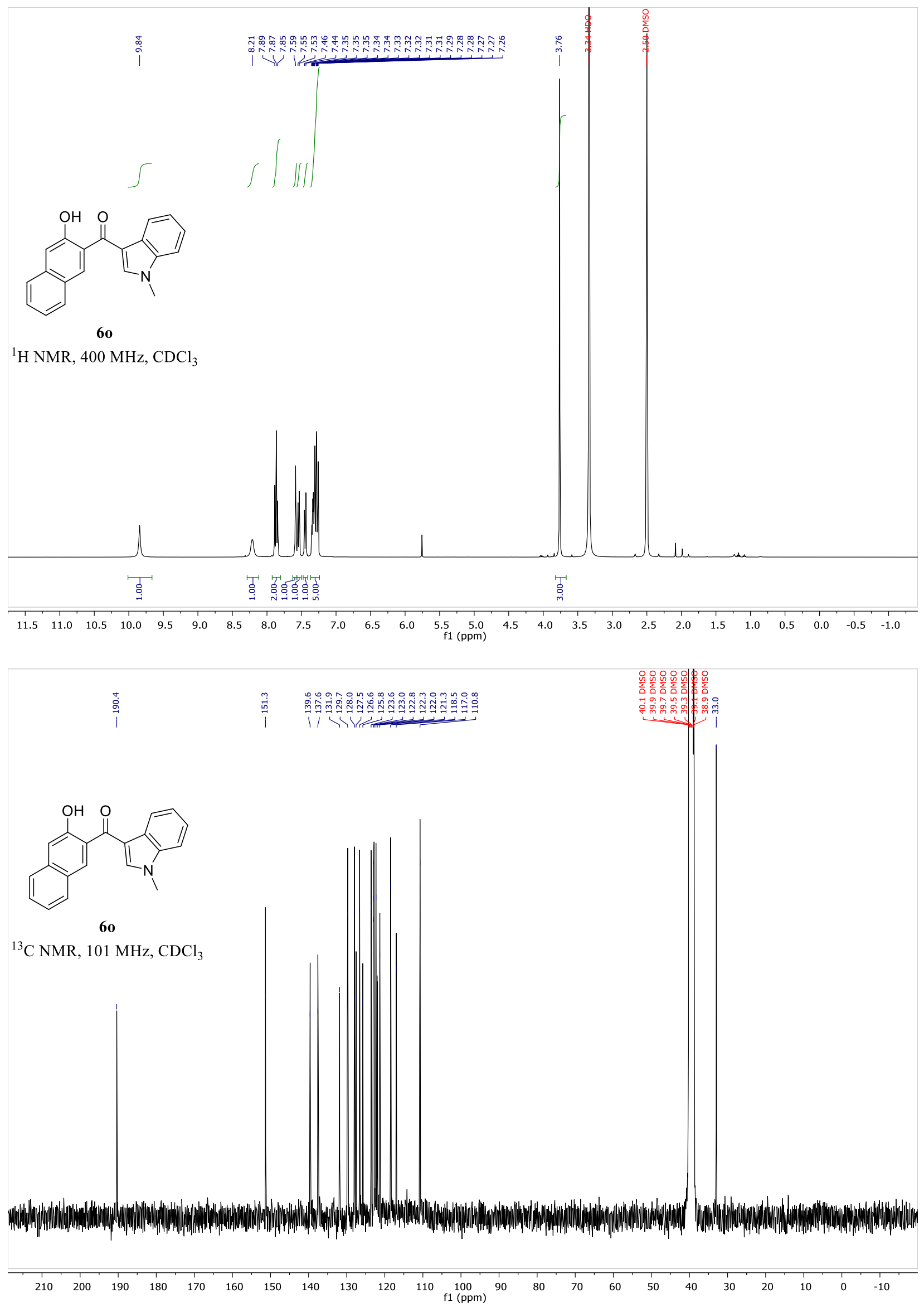

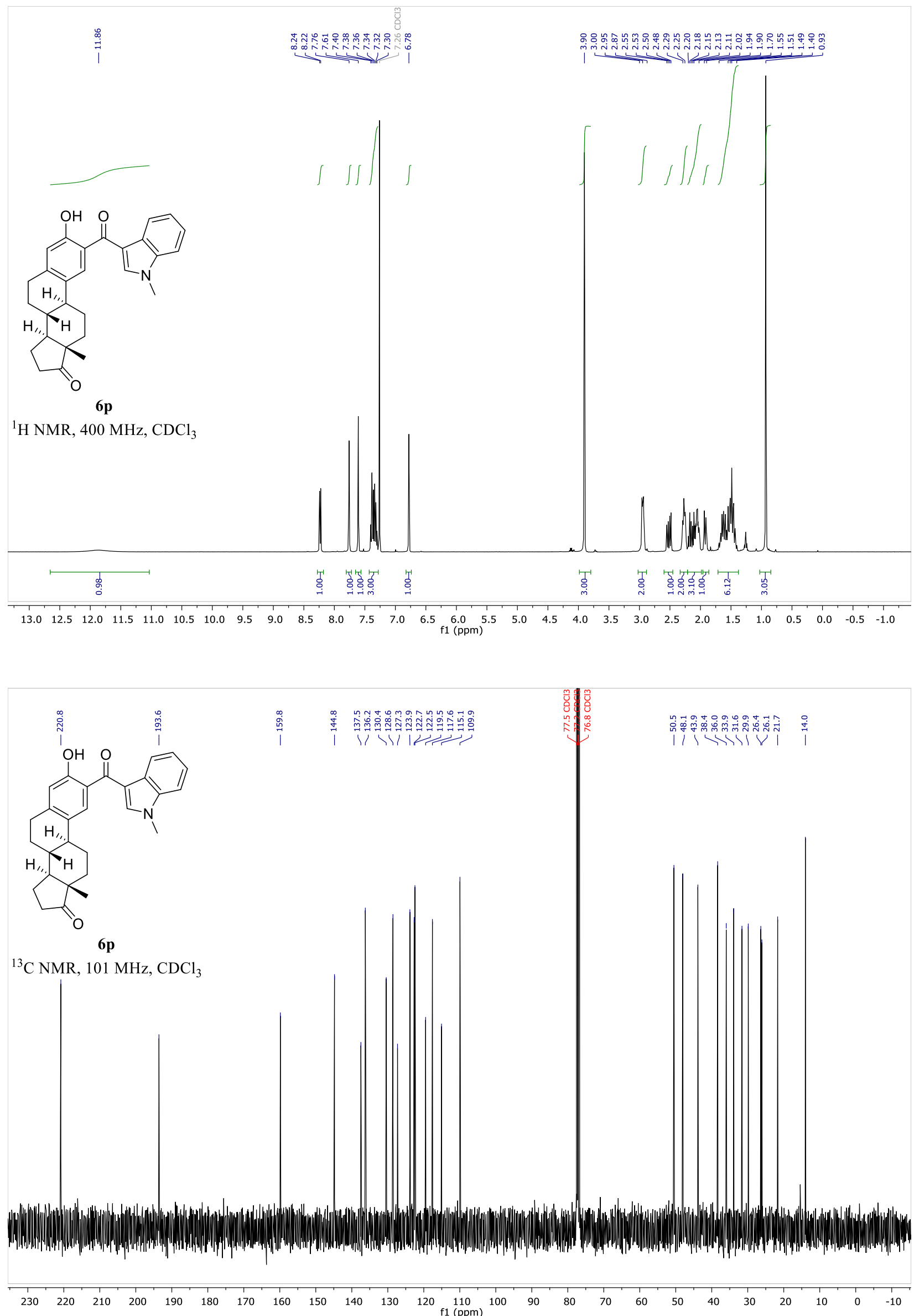

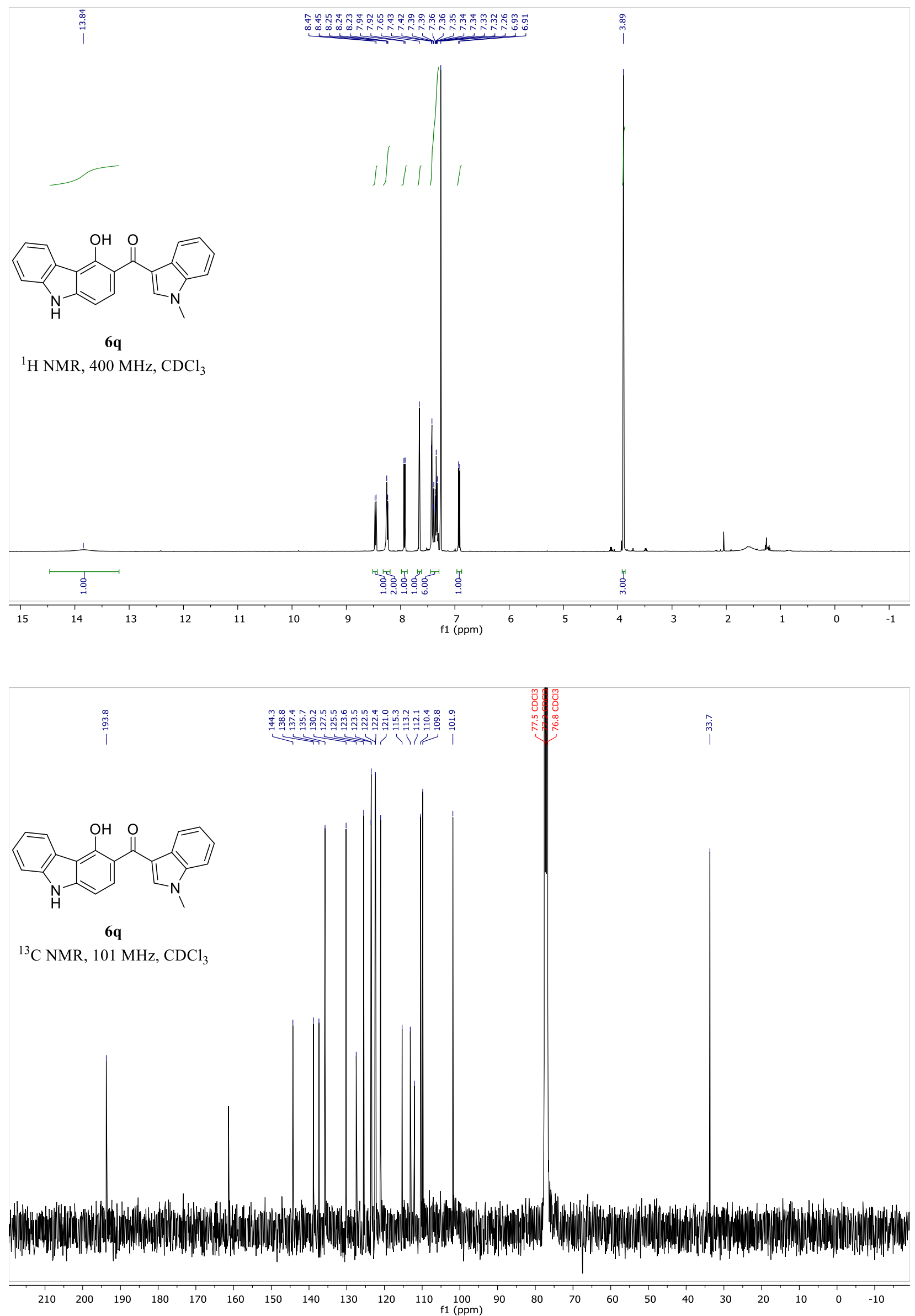

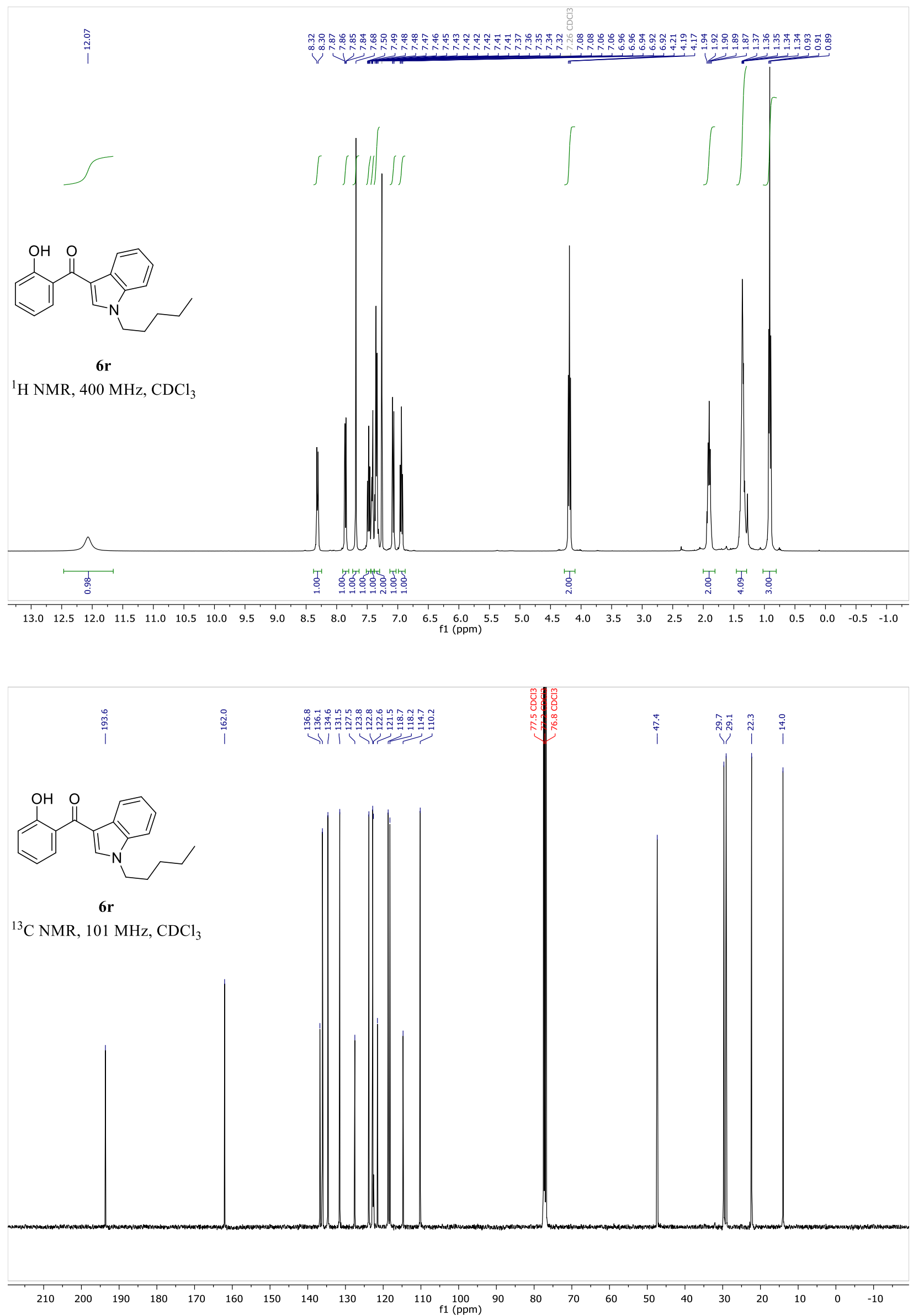

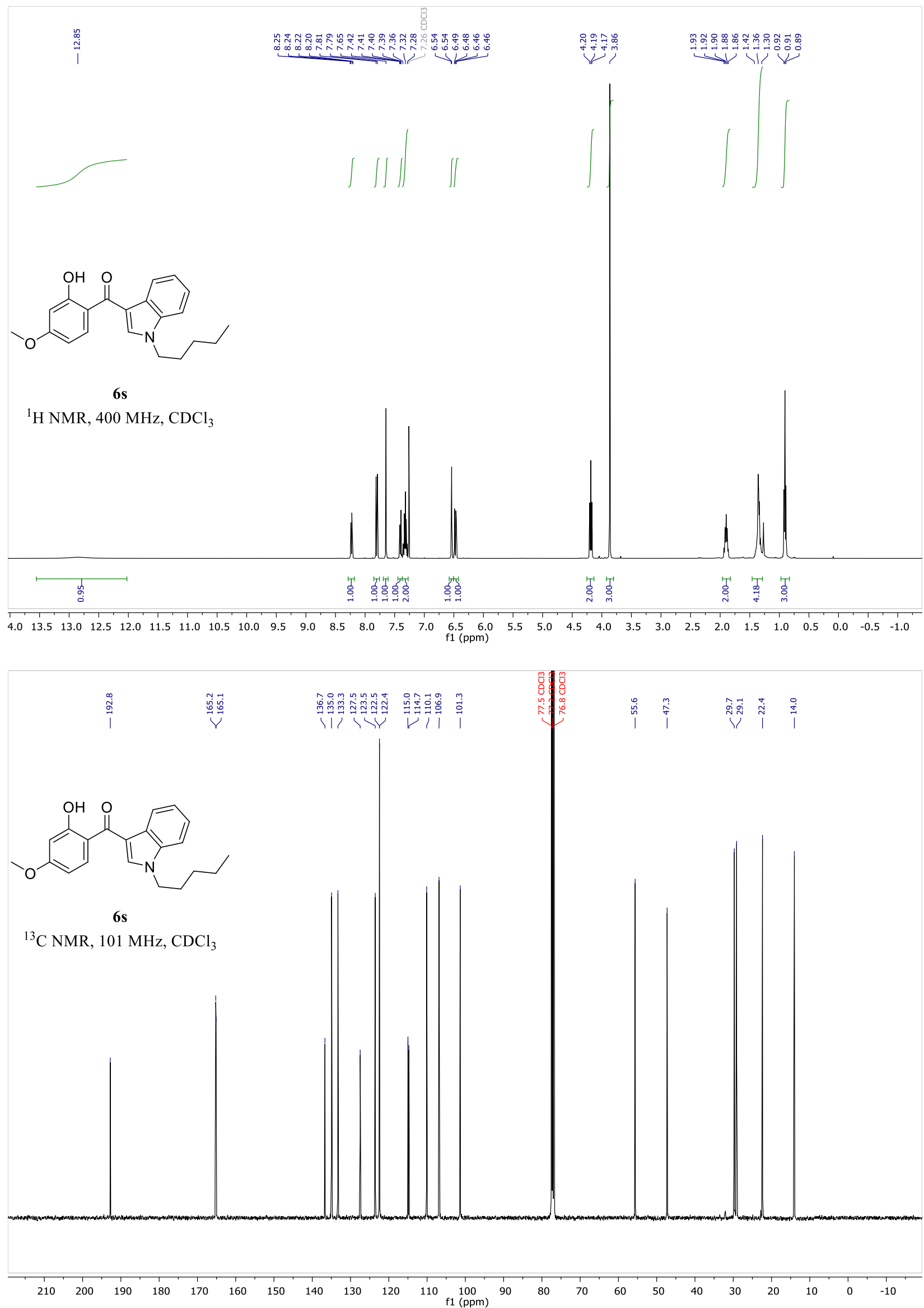

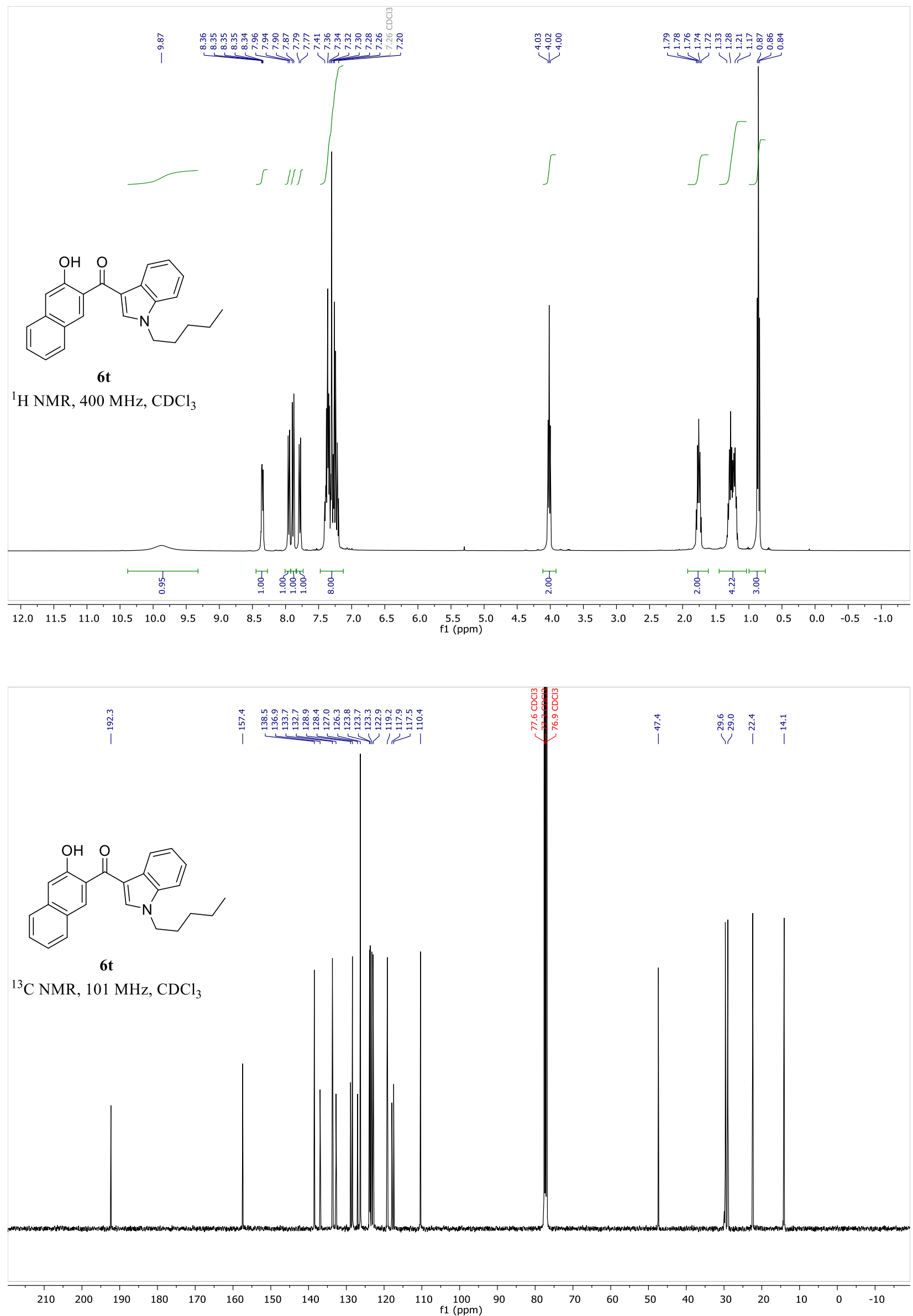

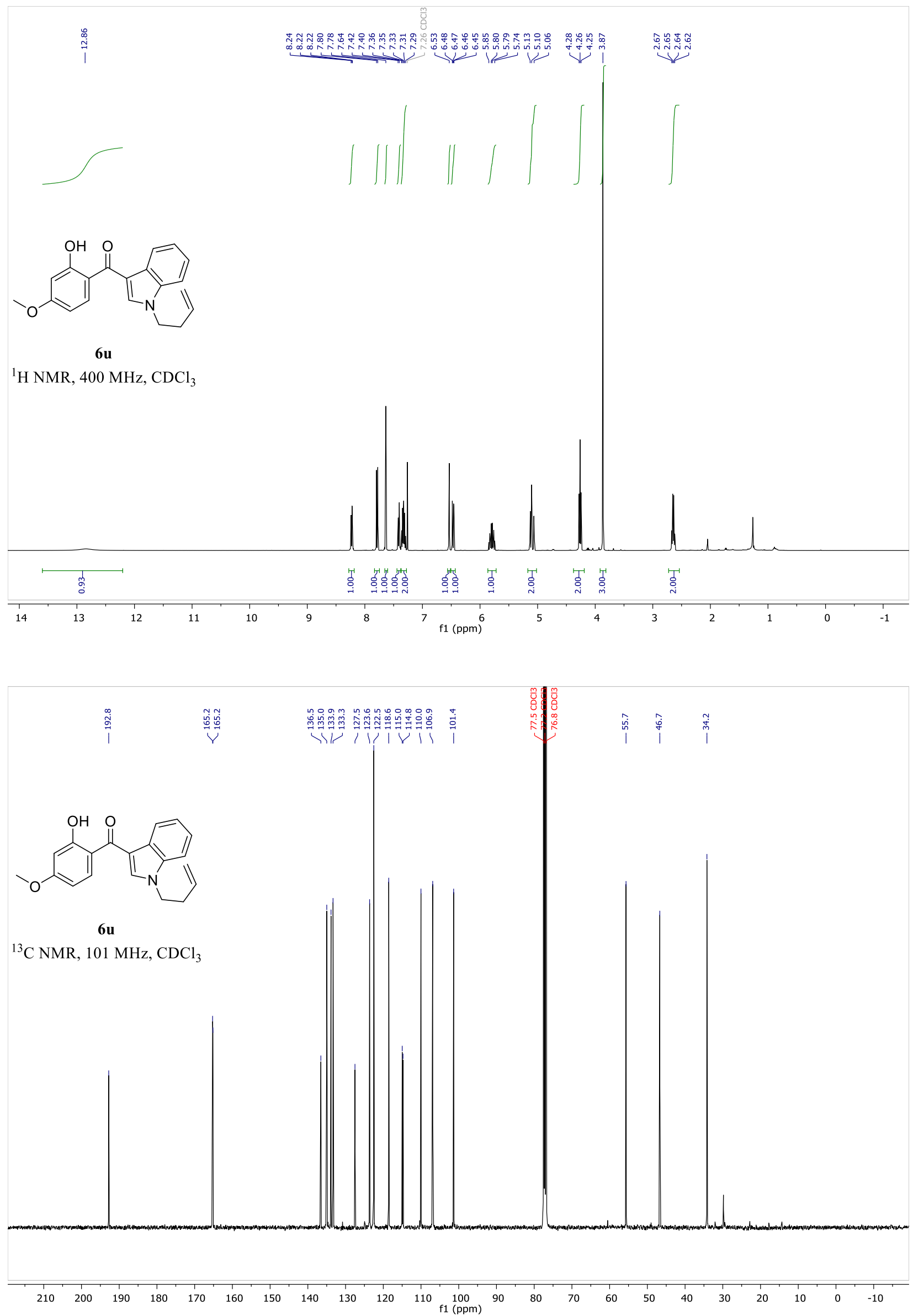

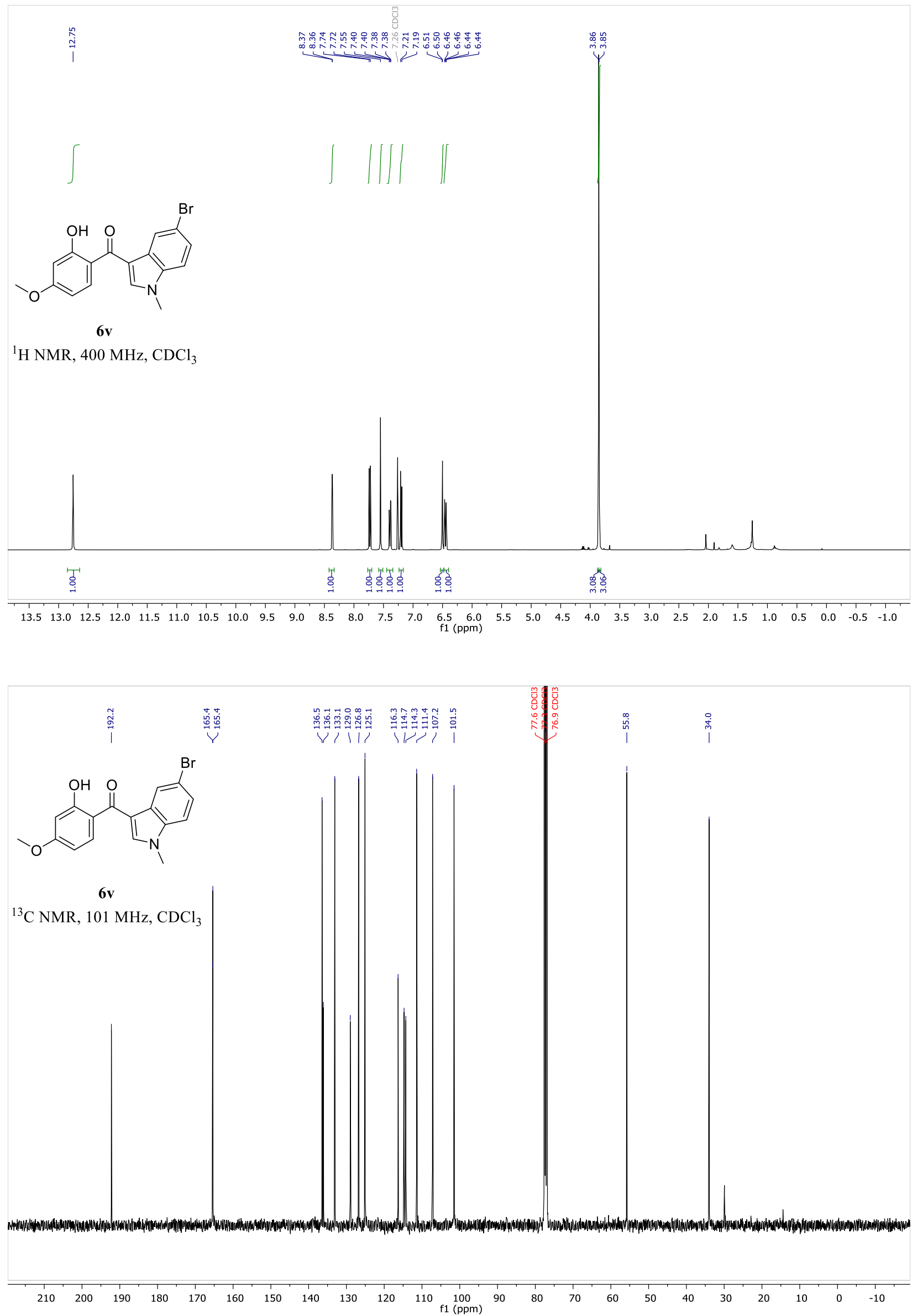

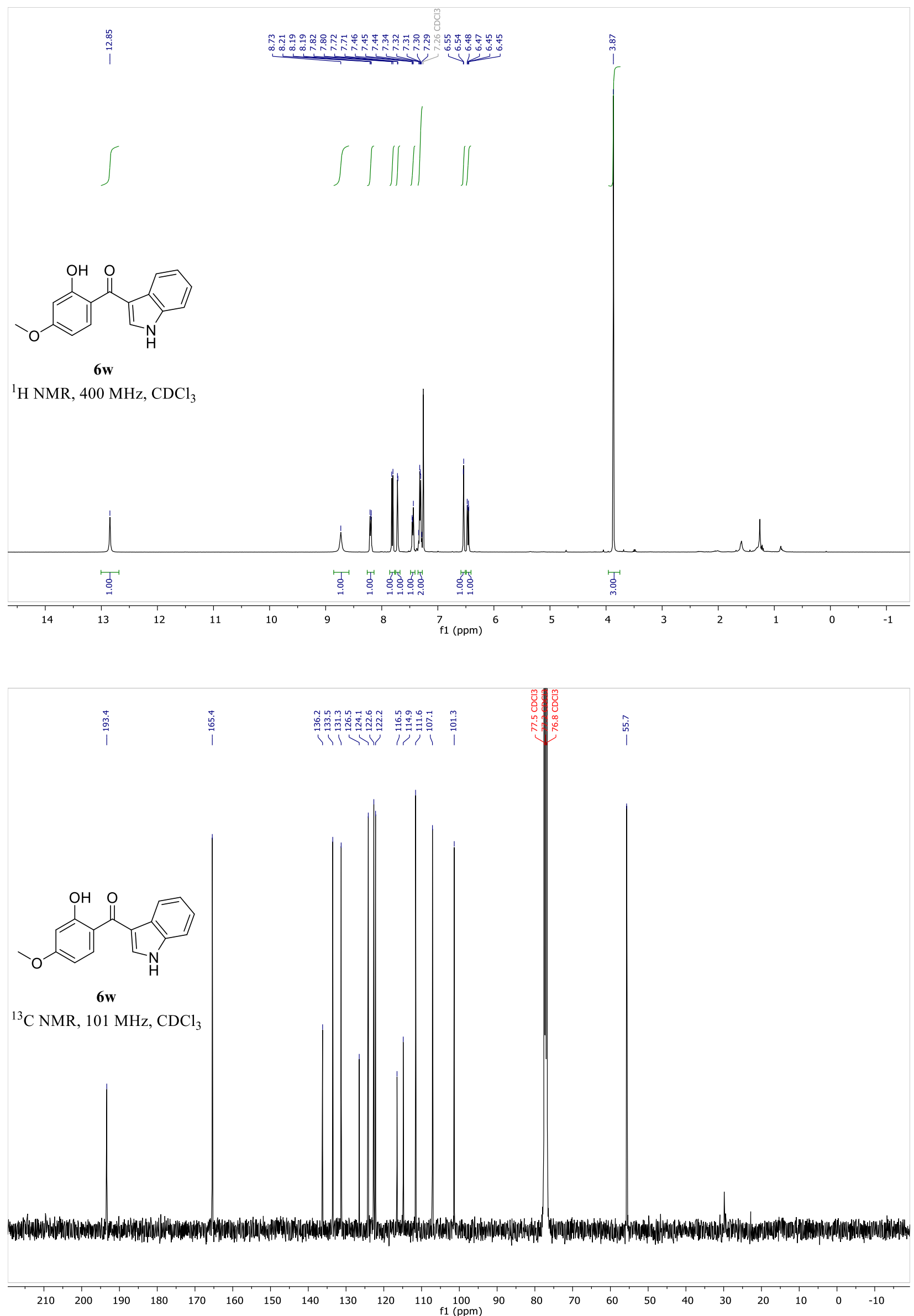

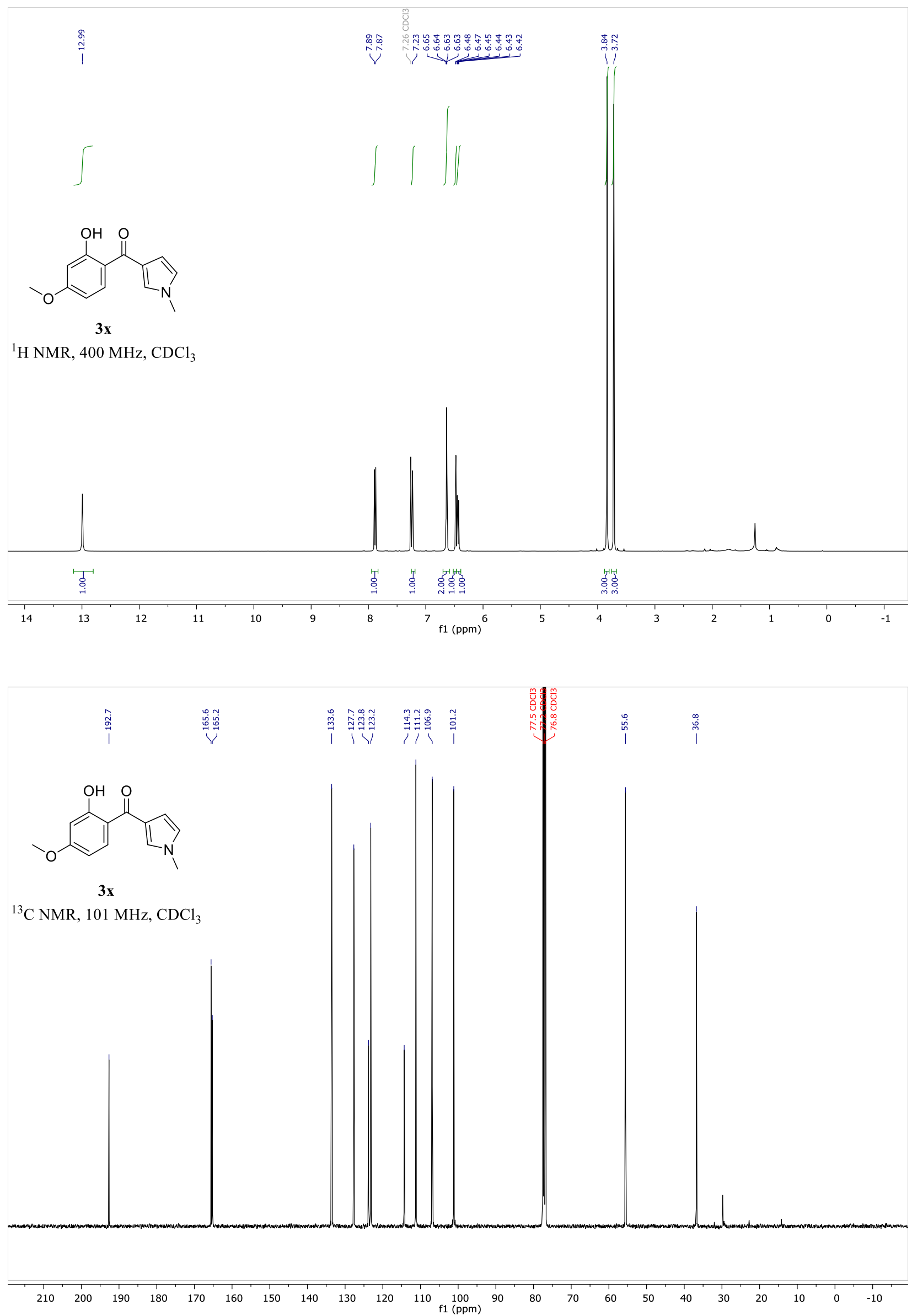

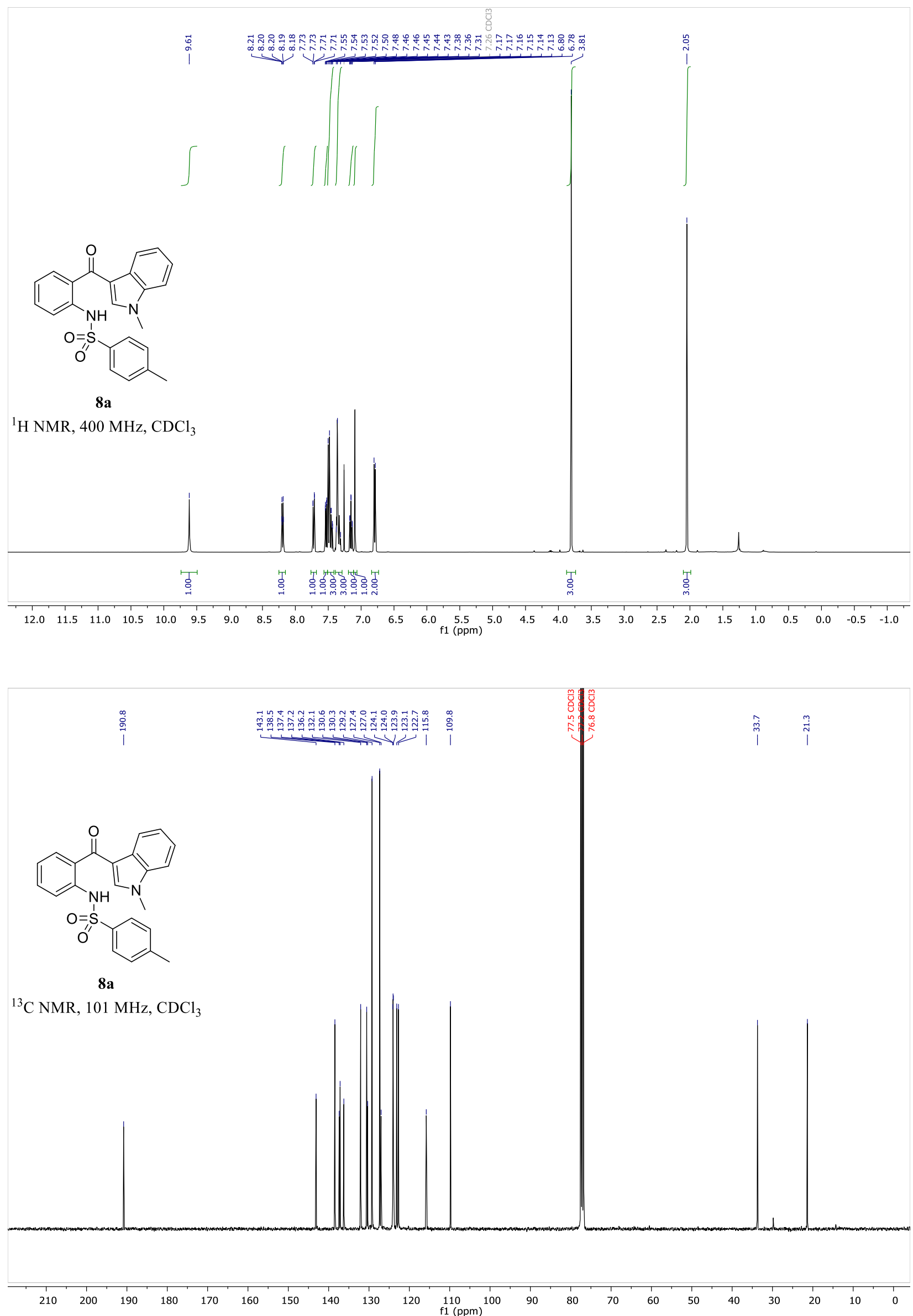

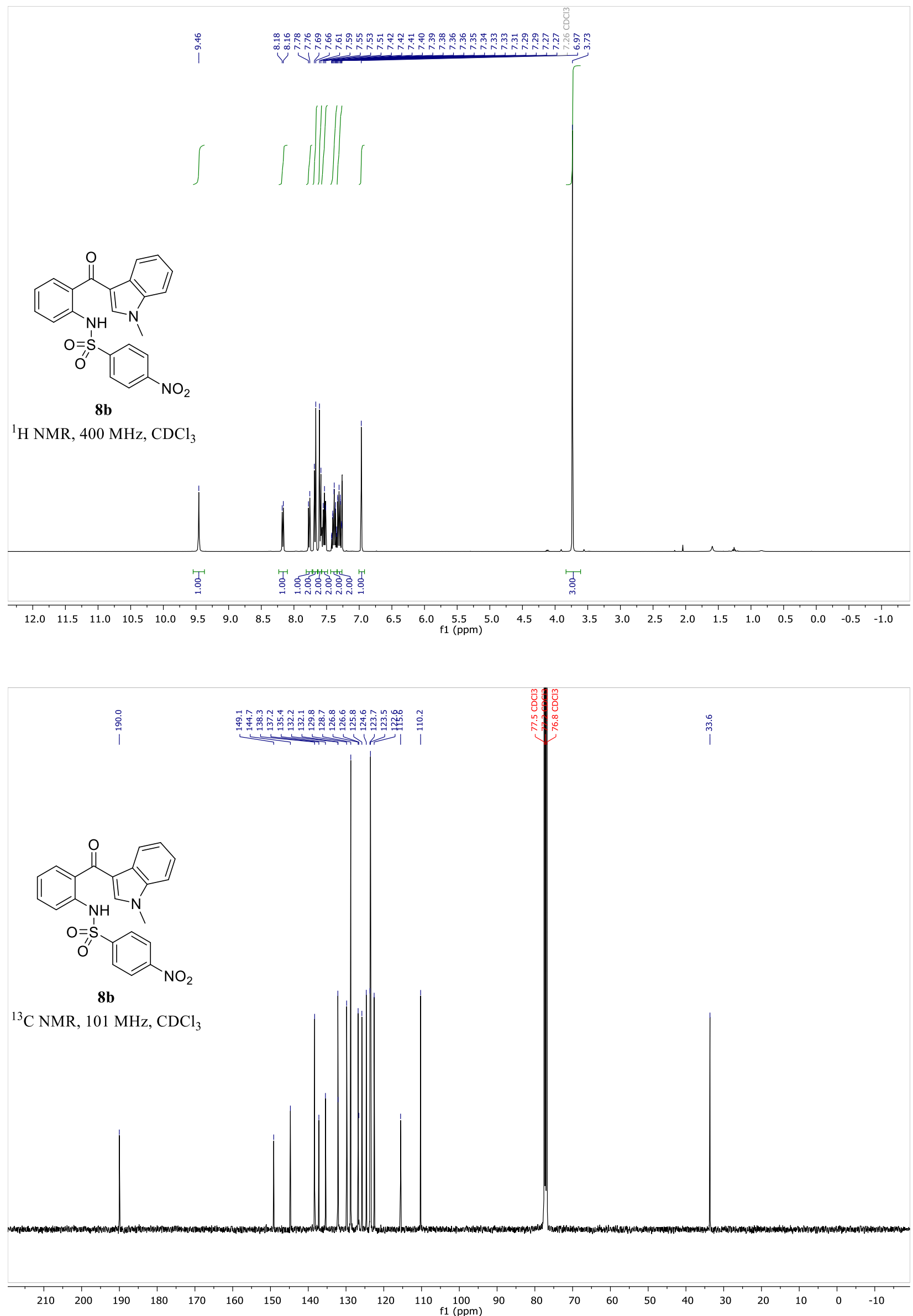

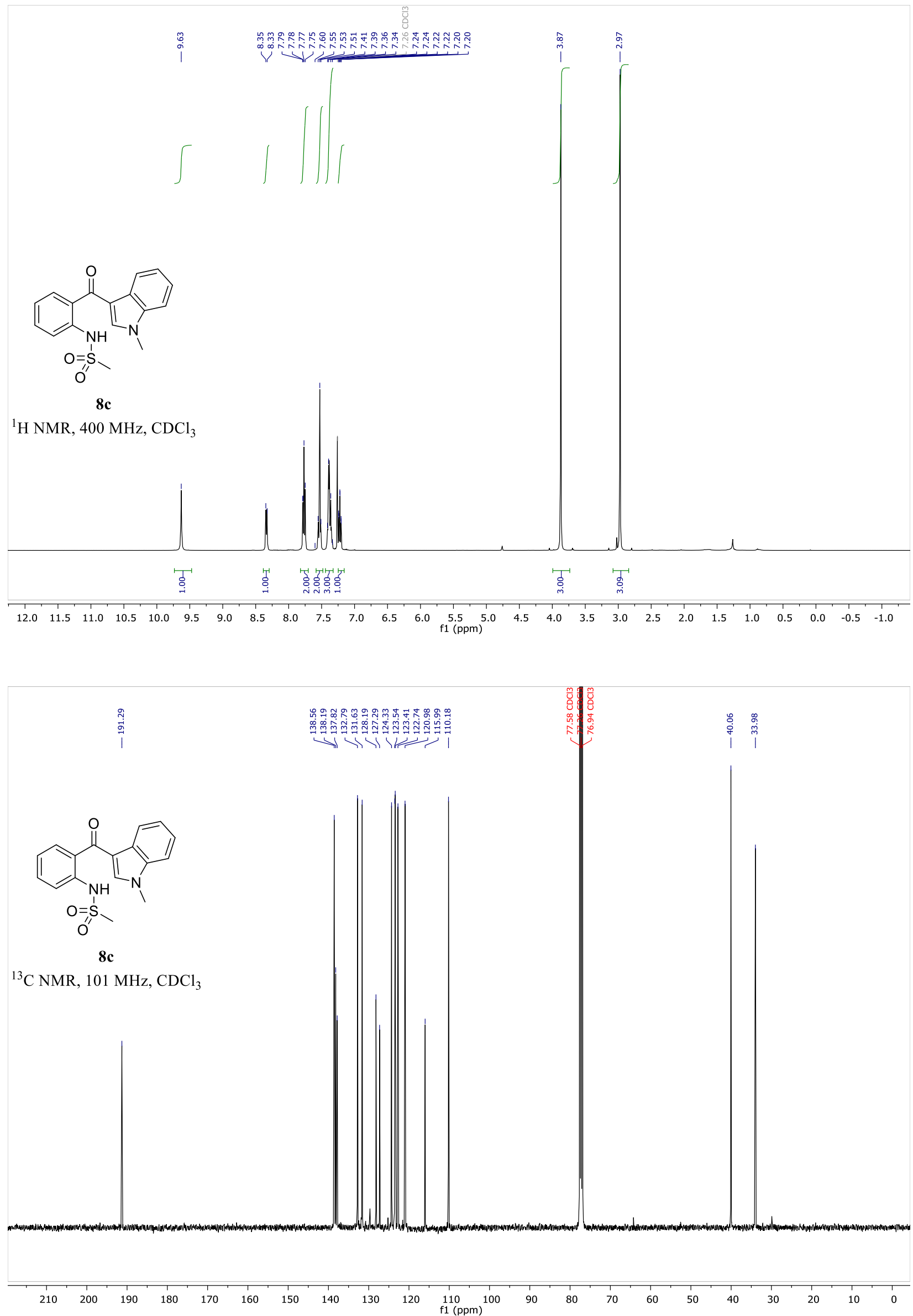

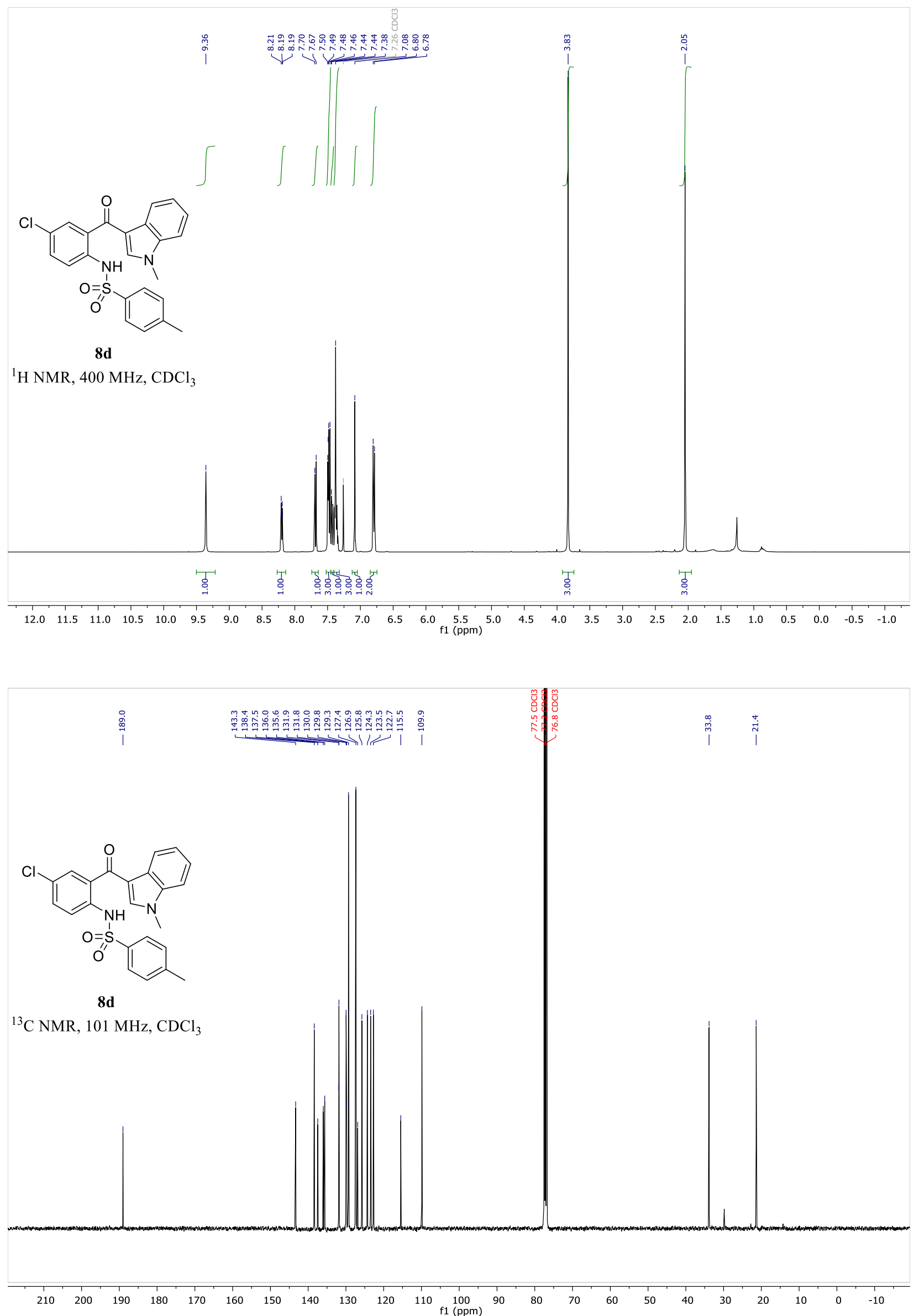

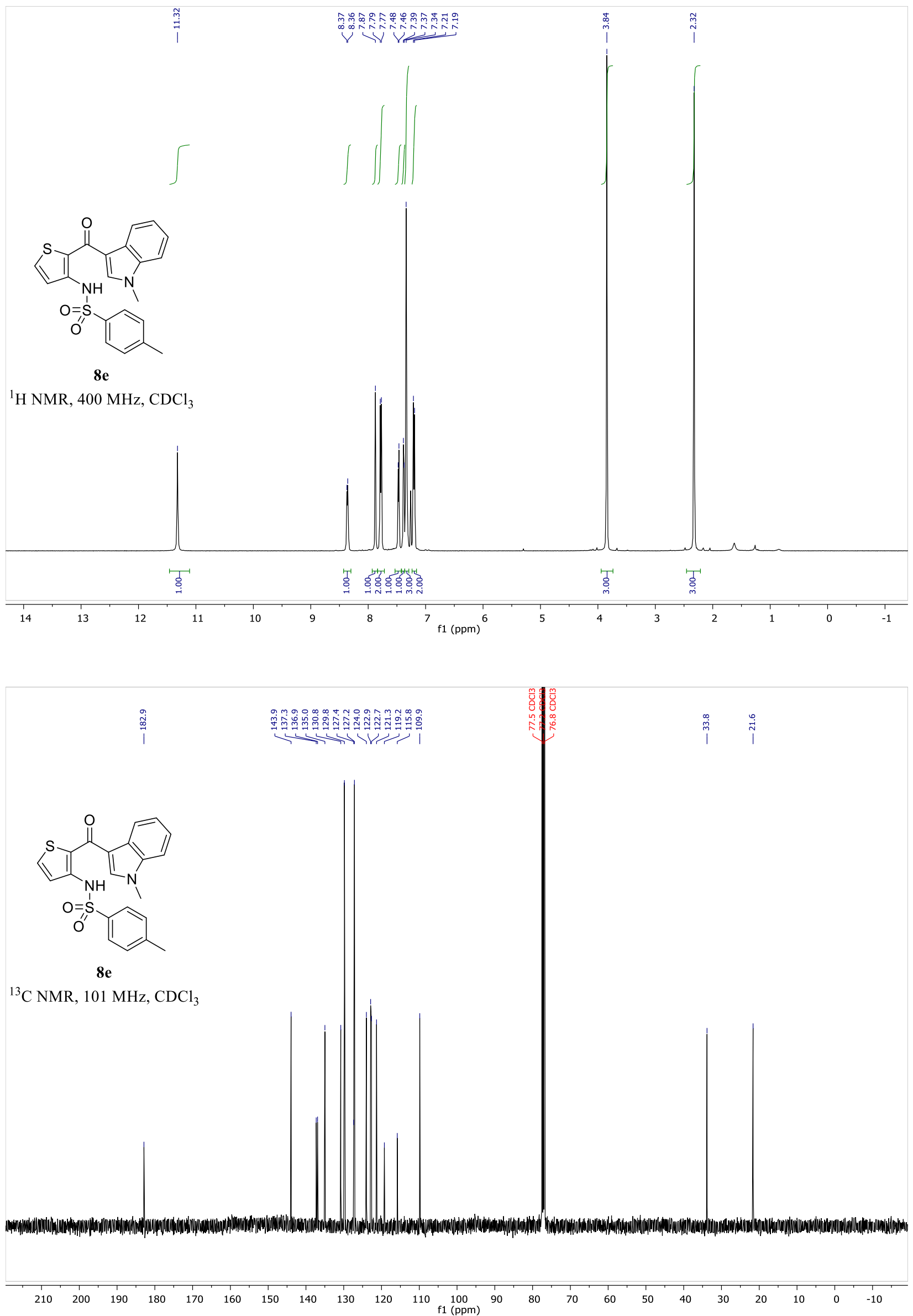

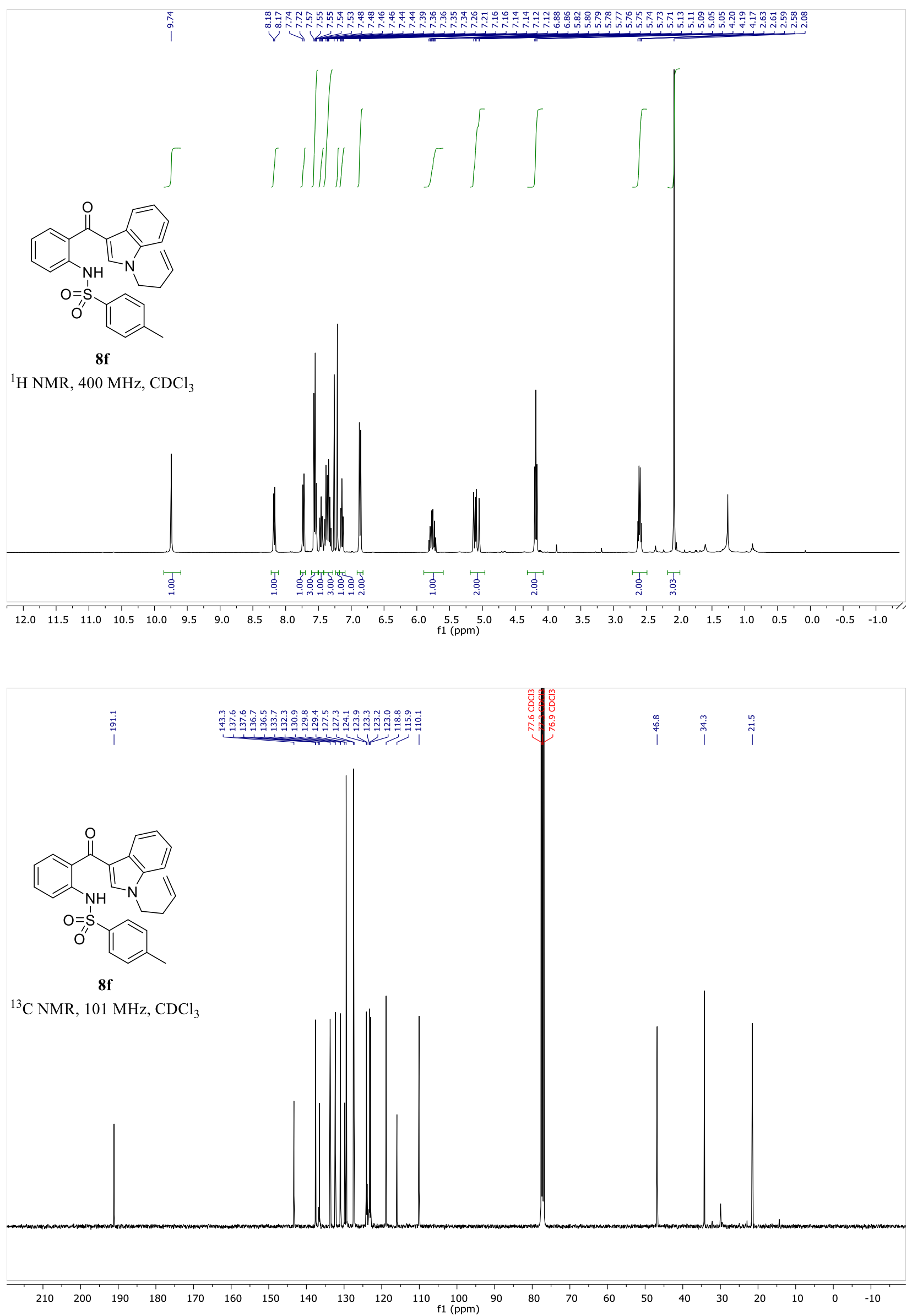

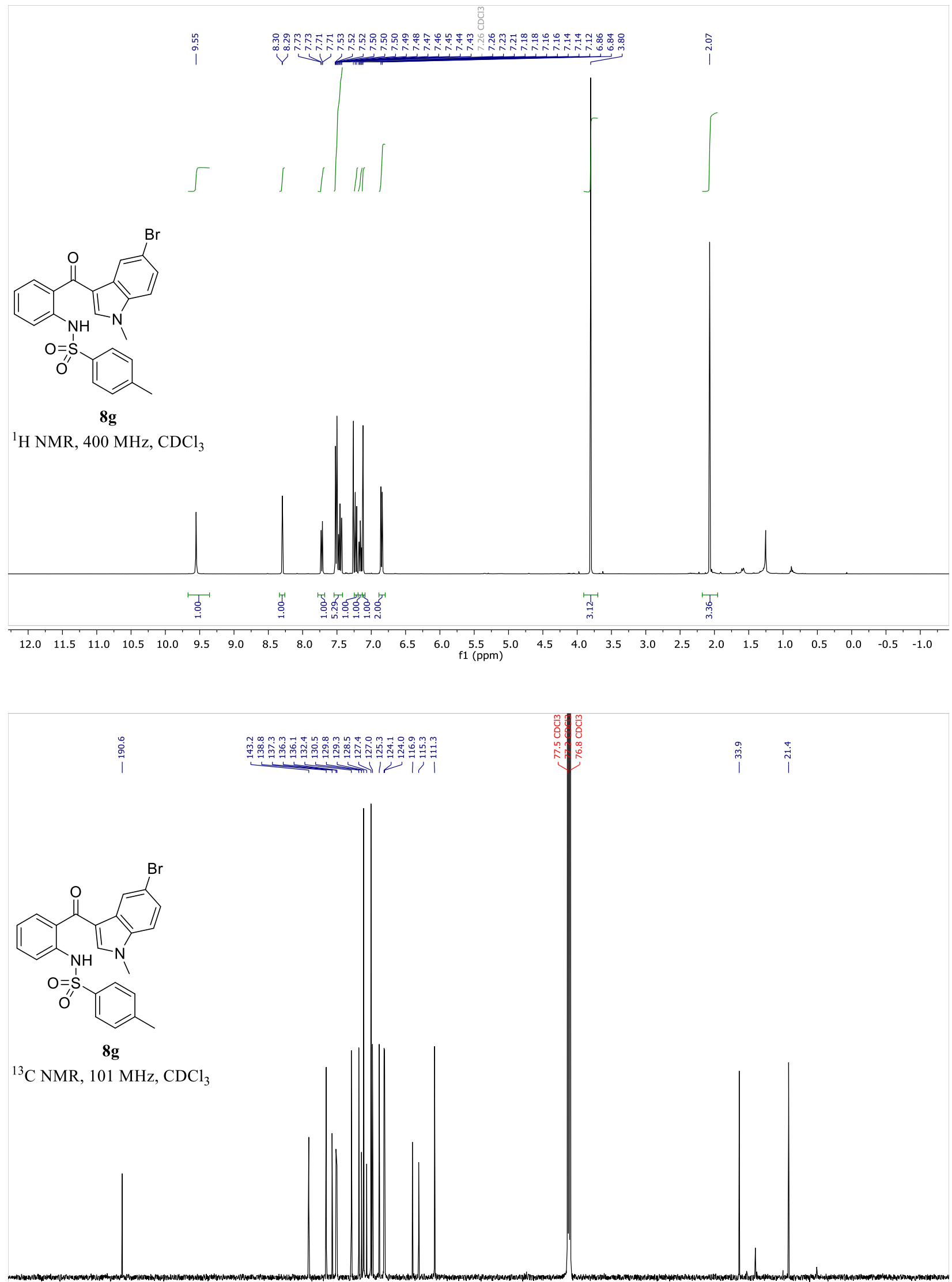

$\begin{array}{llllllllllllllllllllllllllll}210 & 200 & 190 & 180 & 170 & 160 & 150 & 140 & 130 & 120 & 110 & 100 & 90 & 80 & 70 & 60 & 50 & 40 & 30 & 20 & 10 & 0 & -10 & 10\end{array}$ 

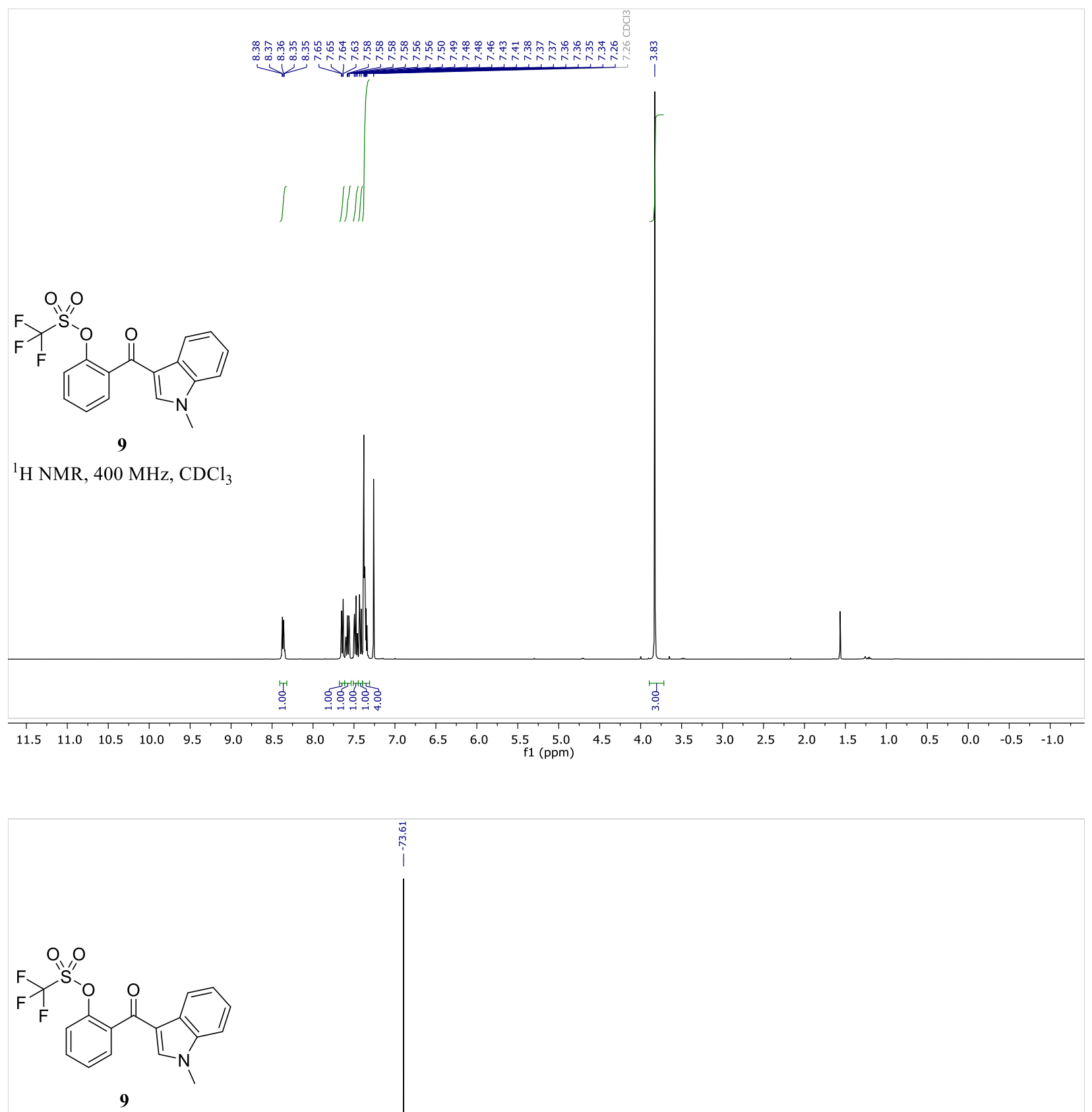

${ }^{19} \mathrm{~F}$ NMR, $376 \mathrm{MHz}, \mathrm{CDCl}_{3}$

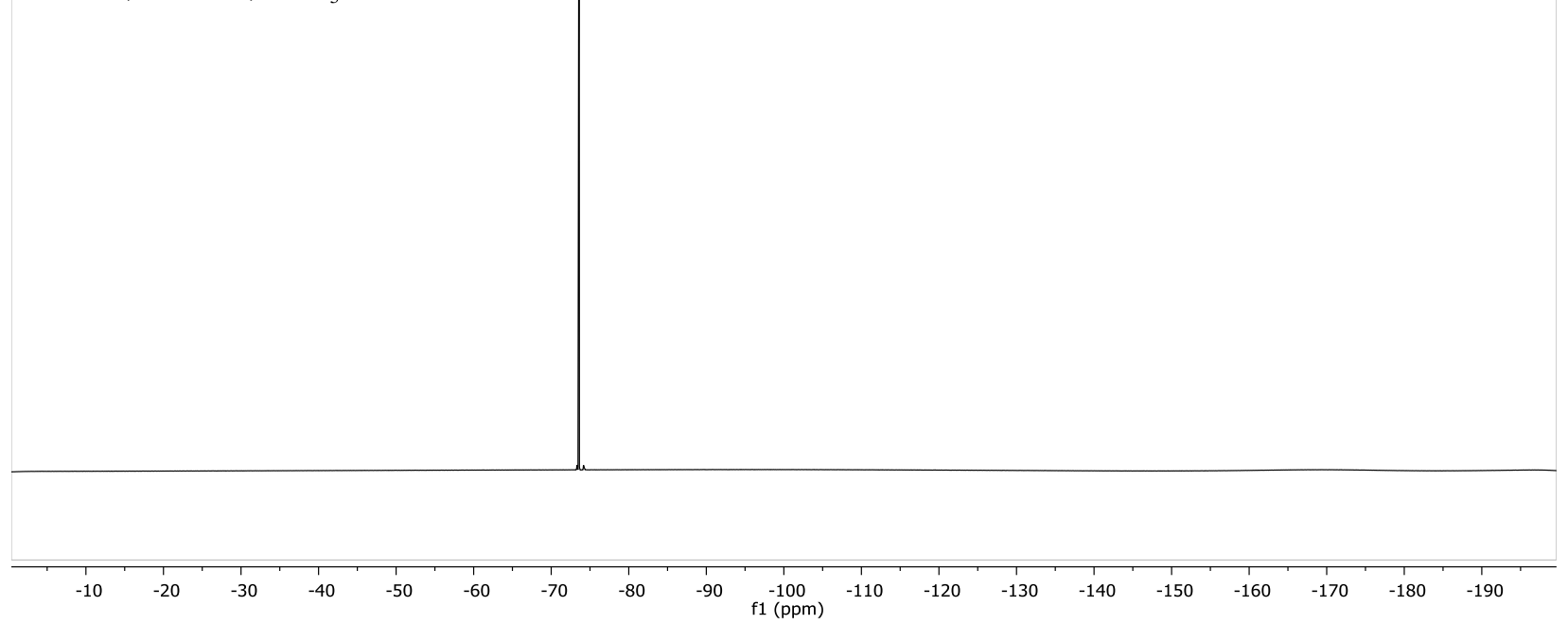




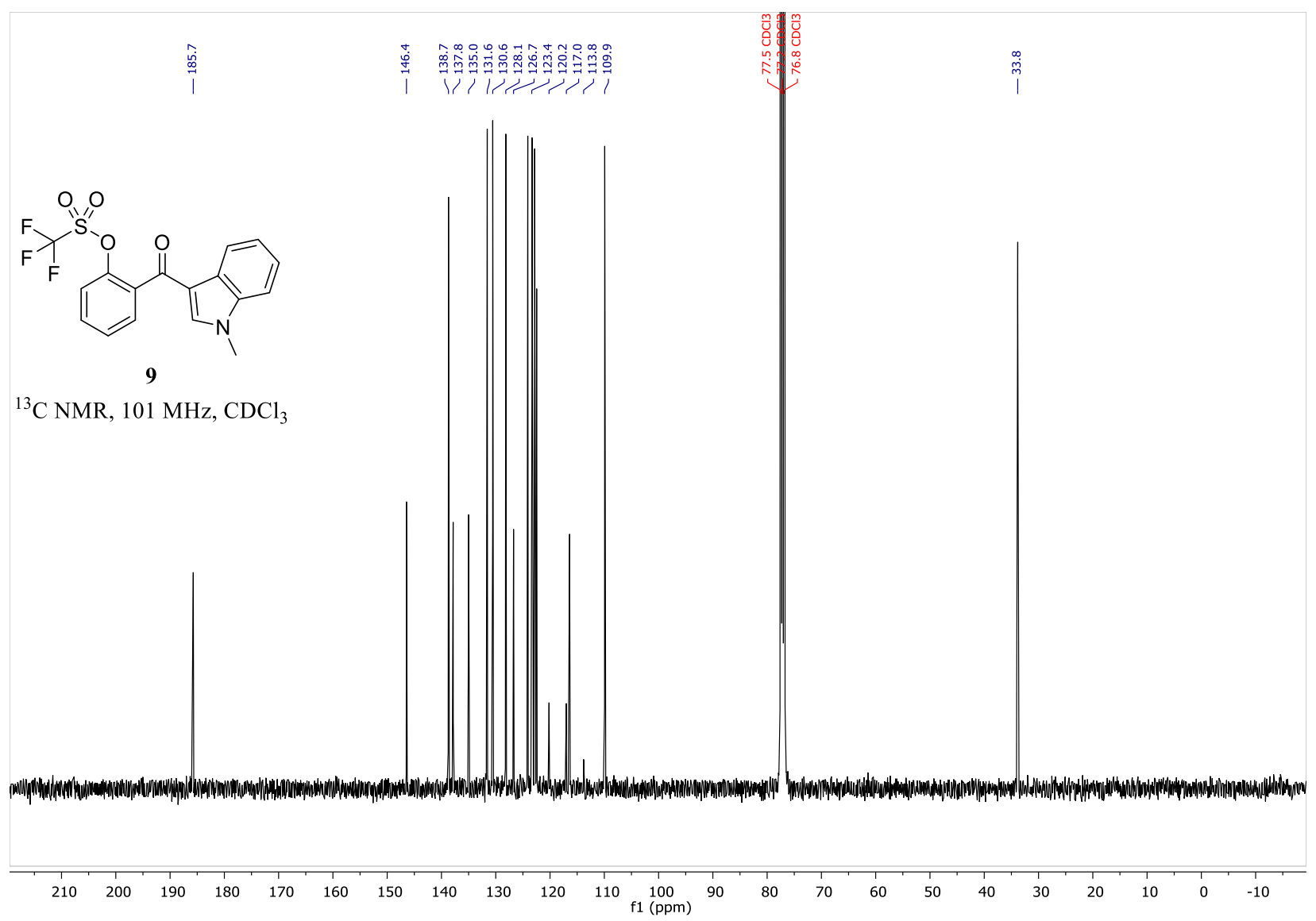



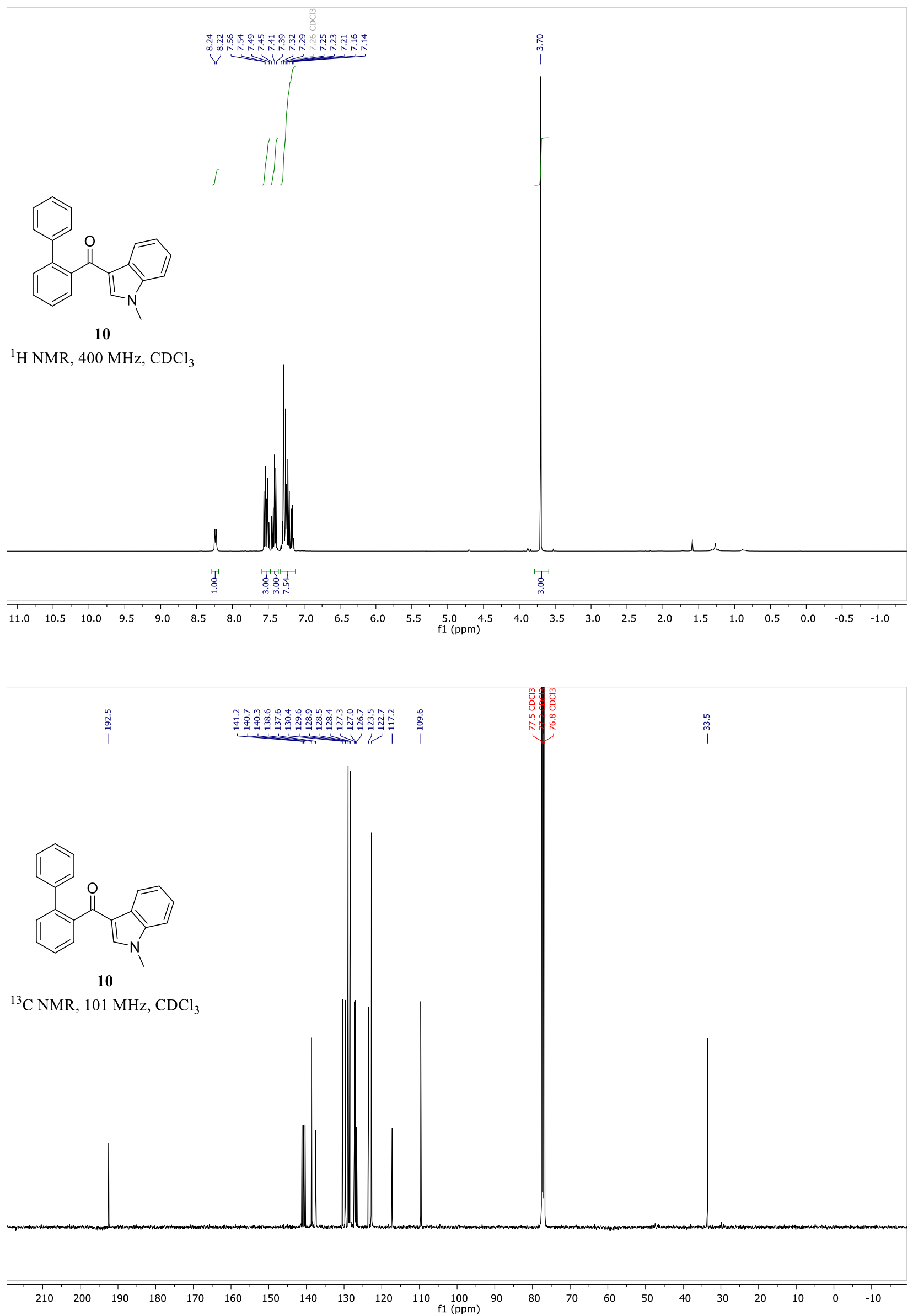

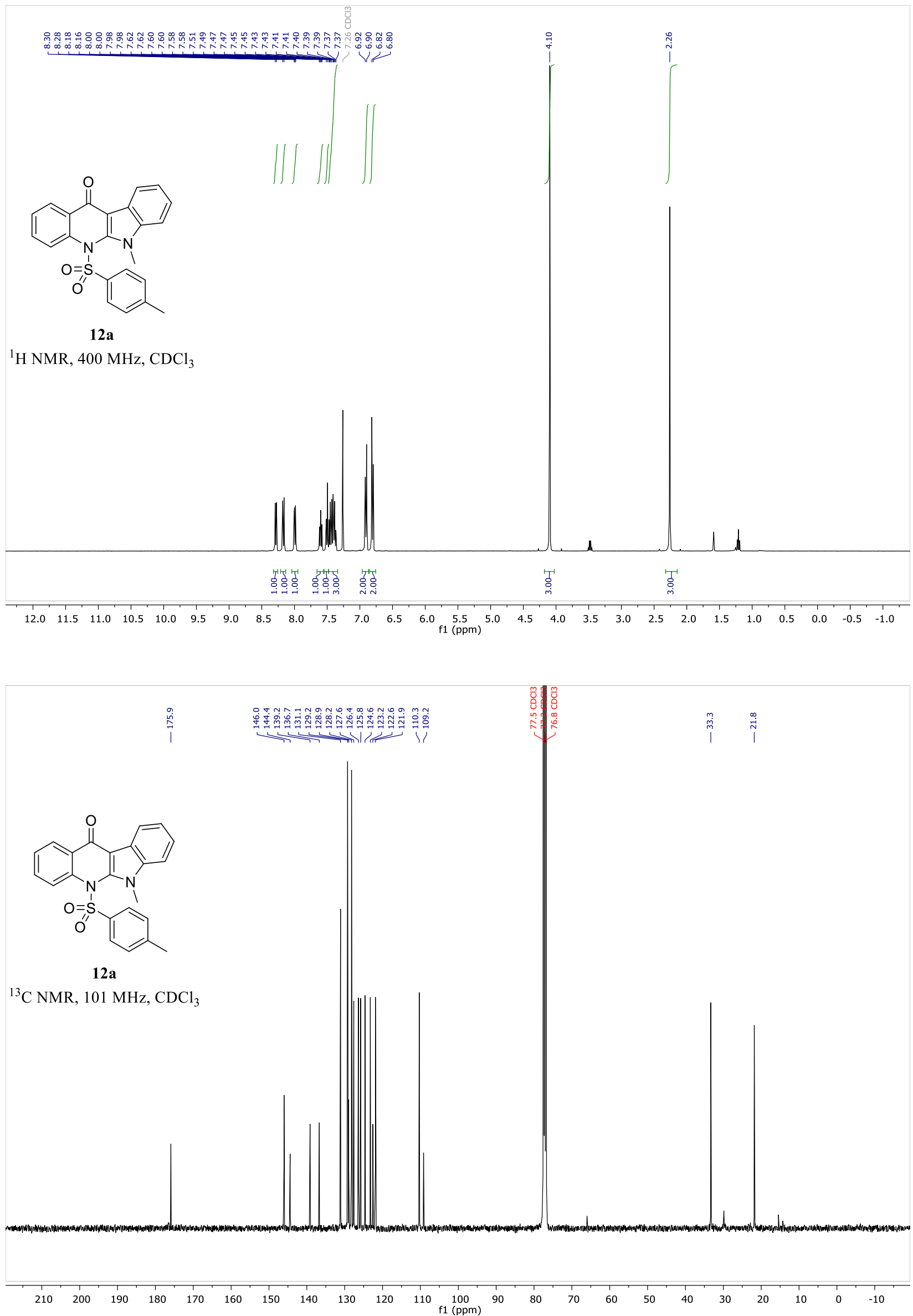

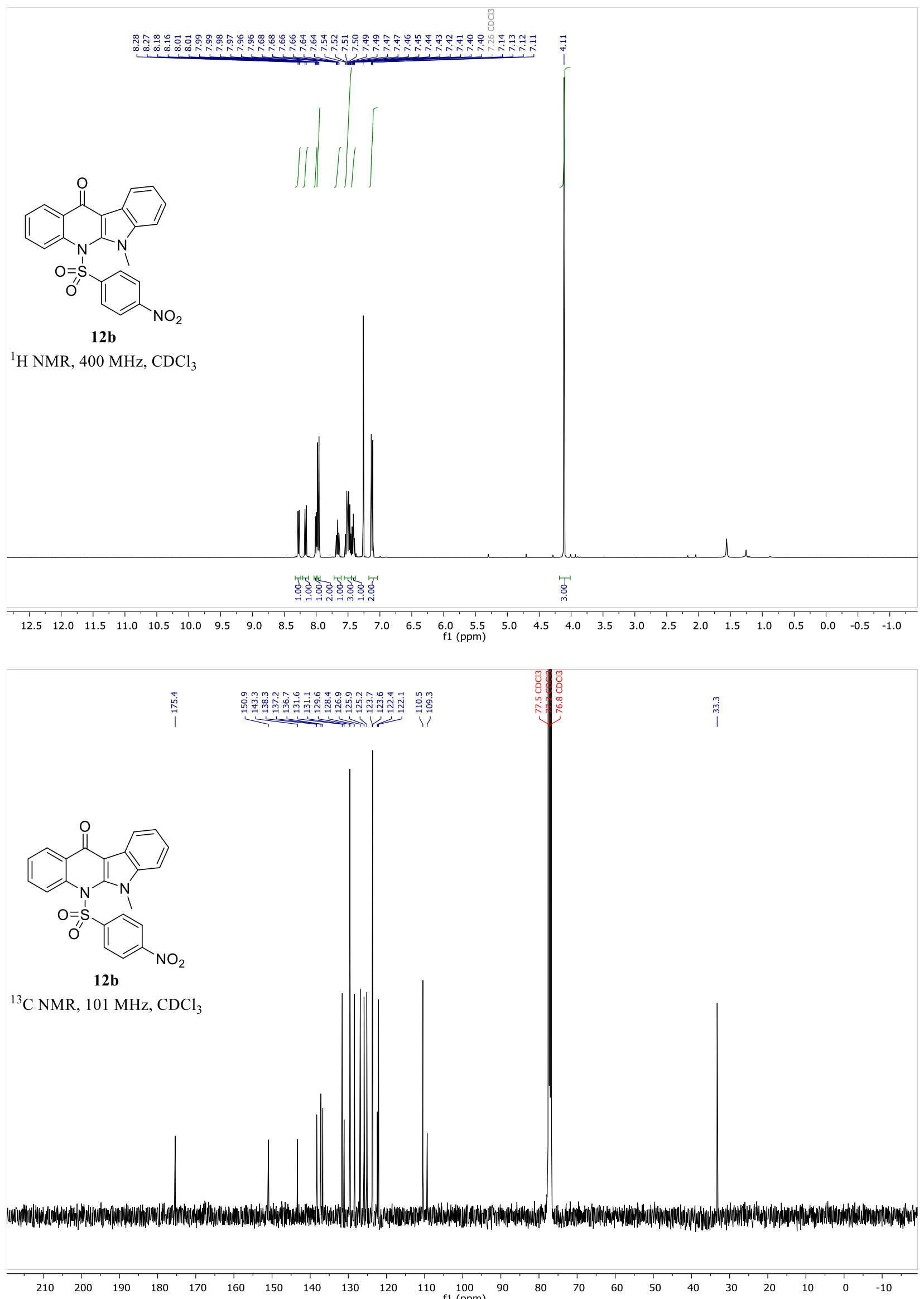

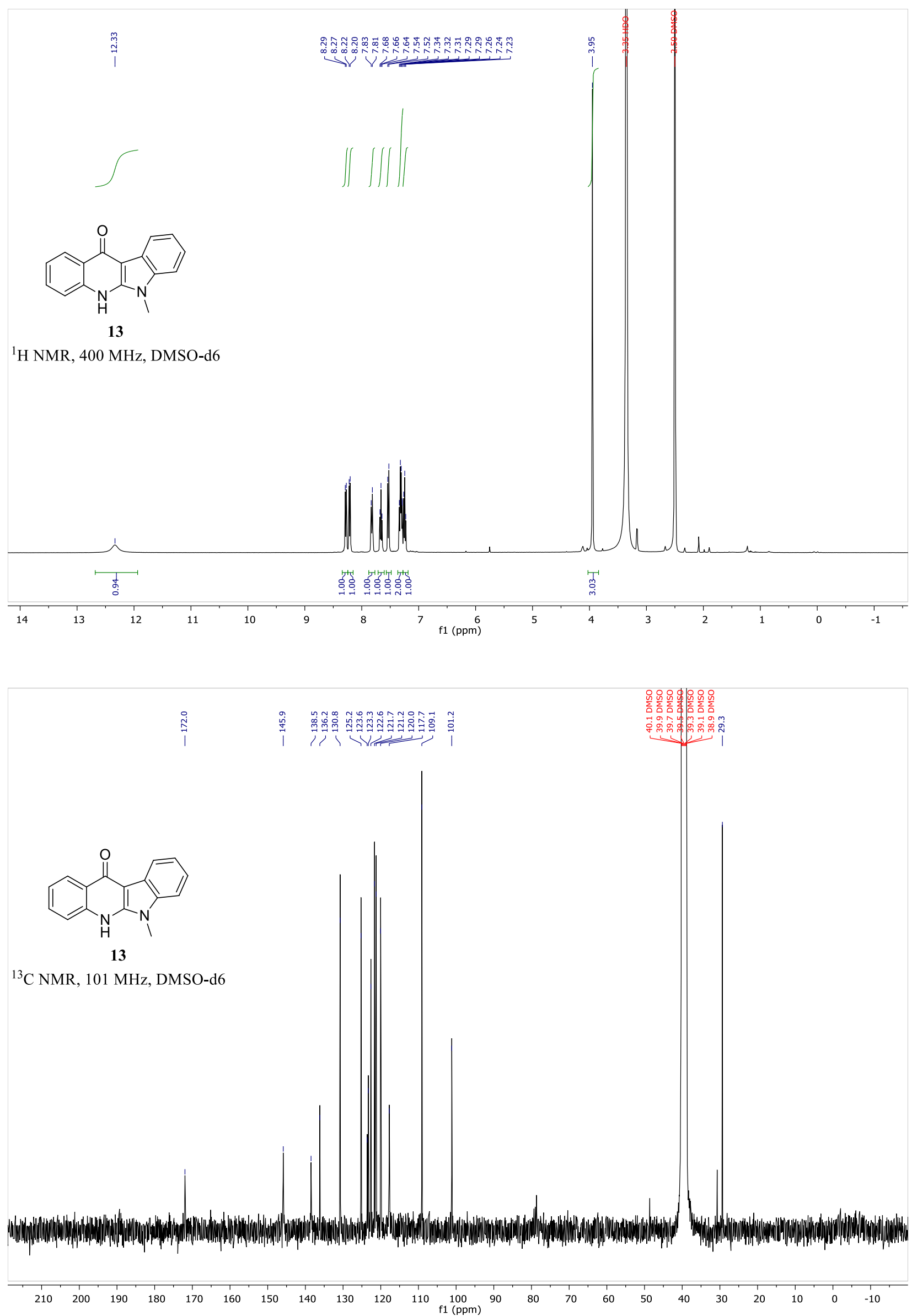

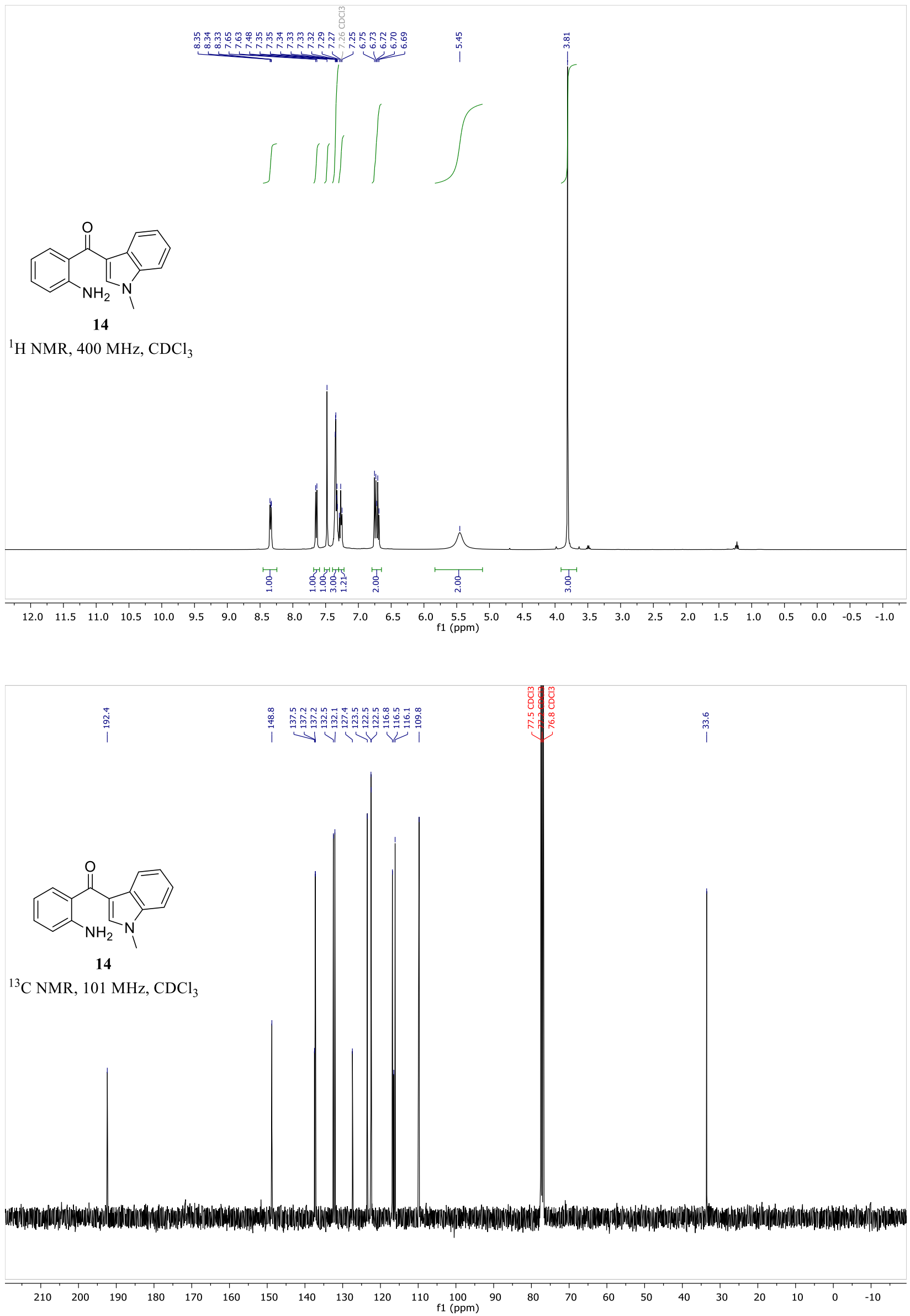